Programa de Doctorado en Biociencias Moleculares

\title{
Dynamics of LDL accumulation \\ leading to atherosclerosis initiation
}

Esmeralda Armando Lewis

Tesis Doctoral-PhD Thesis

Madrid, 2021 

Departamento de Bioquímica Facultad de Medicina

UNIVERSIDAD AUTÓNOMA DE MADRID

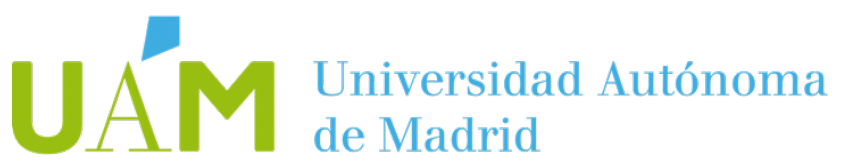

\section{Dynamics of LDL accumulation \\ leading to atherosclerosis initiation}

Memoria de tesis que presenta para optar al título de Doctor por la Universidad Autónoma de Madrid:

\section{Esmeralda Armando Lewis}

Graduada en Biotecnología Molecular

Director de Tesis:

Dr. D. Jacob Fog Bentzon

Centro Nacional de Investigaciones Cardiovasculares Carlos III (CNIC)

Madrid, 2021 

Don Jacob Fog Bentzon, Doctor en Medicina y jefe del grupo "Patología Experimental de la Aterosclerosis" en el Centro Nacional de Investigaciones Cardiovasculares Carlos III (CNIC),

\section{CERTIFICA que:}

Esmeralda Armando Lewis, graduada en Biotecnología Molecular por la "Università degli Studi di Torino" de Turín, Italia, ha realizado bajo su dirección en el Centro Nacional de Investigaciones Cardiovasculares Carlos III (CNIC) el trabajo titulado "Dynamics of LDL accumulation leading to atherosclerosis initiation" para la obtención del grado de Doctor, con Mención Internacional, por la Universidad Autónoma de Madrid. El presente trabajo cumple con todos los requisitos de originalidad y contenido exigidos y contribuye de manera significativa al ámbito de la investigación cardiovascular, por lo que autoriza su presentación para que pueda ser juzgada por el tribunal correspondiente.

Para que así conste y surjan los efectos oportunos, firmo el presente certificado en

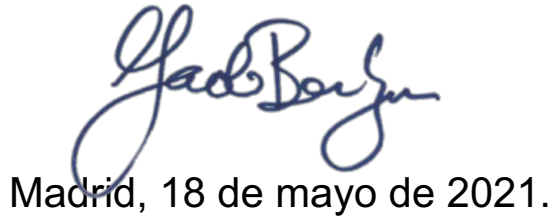

Fdo. Dr. Jacob F. Bentzon 

First and foremost, I wish to express my sincere appreciation to my supervisor, Jacob Fog Bentzon. Thank you for your passionate guidance and support, even during the hardest moments on this path. Your knowledge and your love for science are a true inspiration. I deeply admire how you can cope with supervising each of our projects with such enthusiasm, while at the same time, leading two laboratories in different parts of the world.

A special and deep thank you goes to my colleagues, my companions on this tortuous road. At times it was tough, but then there was always a laugh, a song, a dance, "a tostada con tomate y un piti" and a promise of "we will do it together". To Leti (LR), who is not only a wonderful technician but a wonderful person. You always know what we need and how to help us with a perpetual smile on your face. None of us could have made it without you. With you, Ibi, it was love at first sight. You impersonate happiness and good vibes, and as if that wasn't enough, you're also an amazing scientist. I so much appreciate our time together and your collaboration in this project. Maria, you are a constant in kindness and enthusiasm. Your presence was always a gift. Paula (Claudia, Hermes), you are my left hand. Cariño, I'll probably be deaf in my left ear one day because of you, but it will be worth it. You have been a rock for me during these years, both scientifically and personally. Thank you for putting up with chaotic me (and my brown clothes), and always threatening me with your precise organization (and your latest fashion style). Our shared locker represents us: We are both different and incredibly united. A special thanks goes to Rocio (Wini, Wongui), the person with whom I share Winiland. We often make our own lives harder by having our heads in the clouds, but we always make it at the end (somehow), even more so when we are together. You're my match, my partner in travels, laughter and "Coffee break Signori!". Carlos (Leo, Carlone (B)), the first day we met we already knew each other, and we have been inseparable since then. All these years I always knew that if there was one sure thing, it was us being there for one another. Your ballet, your shopping videos, and your crazy stories are my other half. Without your friendship, the darkest moments of this research would have been so much darker. Thank you. I want to extend the biggest and warmest thanks to all of the members of the Lab of Experimental Pathology of Atherosclerosis for contributing to such a wonderful work environment. Laura, Dani, Ainoa (the queen of immunohistochemistry) and Ester, I cannot picture this road without your continuous moral and scientific support. 
Particular recognition goes to CNIC for support and funding and to all the technical units of CNIC, the animal facility unit, the histopathology unit, the genomic unit, the viral vector unit, the bioinformatics unit, and last, but not least, the microscopy unit. Exceptional regards go to Verónica, able to disentangle any complex microscopy analysis. Your contribution to this project is invaluable.

A special thanks goes to Professor Jens Randel Nyengaard for hosting me at Aarhus University Hospital and introducing us to the wonderful world of electron microscopy. Great recognition goes to the support and help of all my Danish colleagues. Charlotte, Dorte, Gro, Julian, Lisa and Martin, I wish to thank you all for being so welcoming during my time in your lovely country. Mange Tak.

For all the families I have around the world, I am eternally grateful. I would like to specifically say thank you to my family in Madrid: Paola, Eze, Pauli, Álvaro, David, Carlos, Michi, Martín, Jamie, Laura, Cris and Romi. I thank you for making this place home and this period of my life so incredible. Tina, Bea and Vera, I couldn't even imagine these years without you. Our everyday life together, and our incredible travels always gave me strength and happiness. Your support and you all just being yourselves have been pivotal for this project. A deep thanks goes out as well to my family in Aarhus: Rimsha, Toni, Jeorge, Mughda, Mathi, Ivan (Russian guy) and Chiarina made my PhD stay a precious time of my life that I will always look back on with deep affection. My family in Torino offered me love and a home during the manuscript preparation. What a strange year this one was, both challenging and difficult at times, but having given me time with you was irreplaceable. Centova, Dina, Luca (Brescione) and Erika, thank you for being the astounding friends that you are. Special gratitude goes to Luca, my family in Ancona. You kept up with me during this whole process, and I'm pretty sure it wasn't an easy thing to do. Thank you for your continuous affection, support and patience. A very heartfelt thanks goes to my family in my little town, Castiglion Fiorentino, my safe place. Migli, Marti, Torchi (S), Carli, Fede and Noce have always been there for me throughout my life. You are part of me and of everything I do. I wish to show my thanks to my Italo-American family, the best team one could ever get. I am indebted to my grandmother Johnnie, my inspiration, and the strongest woman I know. Every achievement I will ever reach will be in part yours. To my sisters, Giulia, Anna and Rachel, I wish to express gratitude for being my bridges that never burn, and for making me the person I am today. I am thankful every single moment of my life for having you. To my loving mother and father, that 
always supported my dreams, that always helped me walk in whatever path I took, this work is yours. I love you unconditionally. Finally, to all the other members of this strange team, Mike, Vero, Isa, Luca, Chase, Zach, Isaac, Silas and August, I wish to thank you for being my family. Without all of you, this work would not have been possible.

Grazie, Gracias, Tak, Thank you 




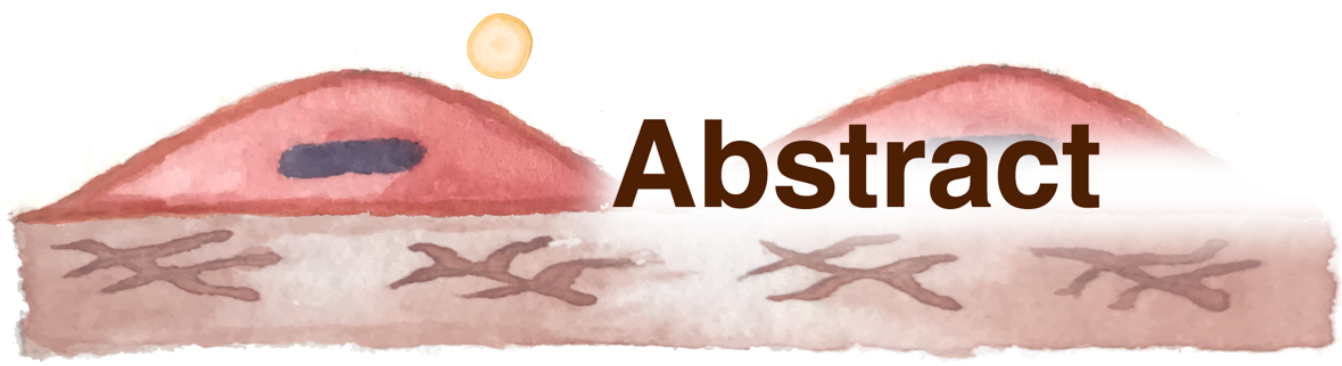


Objective - Atherosclerosis is caused by the accumulation of LDL at atherosclerosissusceptible sites. This requires LDLs to pass through the endothelium and be retained in the arterial intima, but which process is rate-limiting and predicts atherosclerosis has remained controversial. To answer this question, we performed high-resolution mapping for LDL entry and retention in healthy, pre-atherosclerotic and atherosclerotic mouse arteries.

Approach and Results - Rates of LDL entry and retention were measured by injecting fluorescent LDL into mice followed by en face scanning and whole-mount confocal microscopy of the aortic arch after 1 hour (entry) and 18 hours (retention). Measurements were performed in groups of pre-atherosclerotic mice after short-term hypercholesterolemia and in normocholesterolemic mice with a similar clearance rate of the fluorescent LDL probe. We found that rates of LDL entry and retention under normal and pre-atherosclerotic conditions are dissociated and divide the aortic arch into three zones: an outer arch zone with low LDL entry and LDL retention, and subdivisions of the inner arch region consisting of a border zone with high LDL entry and high LDL retention and a central inner arch zone with intermediate LDL entry and saturable LDL retention. These characteristics predicted susceptibility to atherosclerosis, which was low in the outer zone, high in the border zone, and intermediate in the central inner zone. Saturation of LDL retention in the central inner zone was intrinsic to the arterial wall and was lost with the onset of atherosclerosis.

Conclusion - Rates of LDL entry and retention in arteries are dissociated and provide distinct information on atherosclerosis susceptibility. Combined, they predict where and when atherosclerosis develops in the mouse aortic arch. 


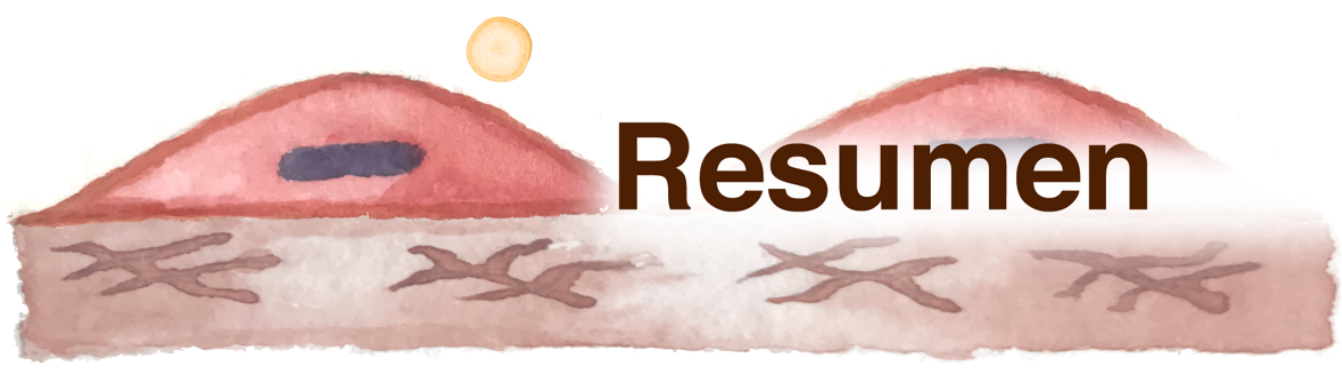


Objetivo - La aterosclerosis está causada por la acumulación de partículas de LDL en regiones de la pared arterial más susceptibles a esta enfermedad. Para ello, se requiere que las LDL atraviesen el endotelio y sean retenidas en la íntima arterial, pero qué proceso actúa como limitante y es capaz de predecir el desarrollo de la placa sigue resultando controvertido. Para responder a esta pregunta, realizamos un mapeo de alta resolución a partir de la entrada y de la retención de LDL en arterias de ratón sanas, pre-ateroscleróticas y ateroscleróticas.

Métodos y Resultados - Se midieron las tasas de entrada y de retención de LDL inyectando LDL fluorescente en ratones. A continuación, se realizó un escaneo en face de la aorta, así como microscopía confocal de preparaciones del arco aórtico completo después de 1 hora (entrada) y 18 horas (retención) de circulación del LDL. Las mediciones se realizaron en grupos de ratones pre-ateroscleróticos después de un breve estado de hipercolesterolemia, y en ratones normocolesterolémicos con una ratio similar de eliminación de la sonda de LDL fluorescente. Encontramos que las tasas de entrada y de retención de LDL en condiciones normales y preateroscleróticas se disocian y dividen el arco aórtico en tres zonas: una zona del arco externo con baja entrada y retención de LDL, y subdivisiones de la región del arco interno, una zona fronteriza con alta entrada y retención de LDL, y una zona central interna con entrada moderada de LDL y retención saturable. Estas características predijeron la susceptibilidad a la aterosclerosis, que fue baja en la región externa, alta en la región fronteriza e intermedia en la zona interna central. La saturación de la retención de LDL en la zona interna central era intrínseca a la pared arterial y se perdió con el inicio de la aterosclerosis.

Conclusión - Las tasas de entrada y de retención de partículas de LDL en la arteria están disociadas y proporcionan información clara sobre la susceptibilidad a la aterosclerosis. Combinadas, permiten predecir dónde y cuándo se desarrolla la aterosclerosis en el arco aórtico del ratón. 




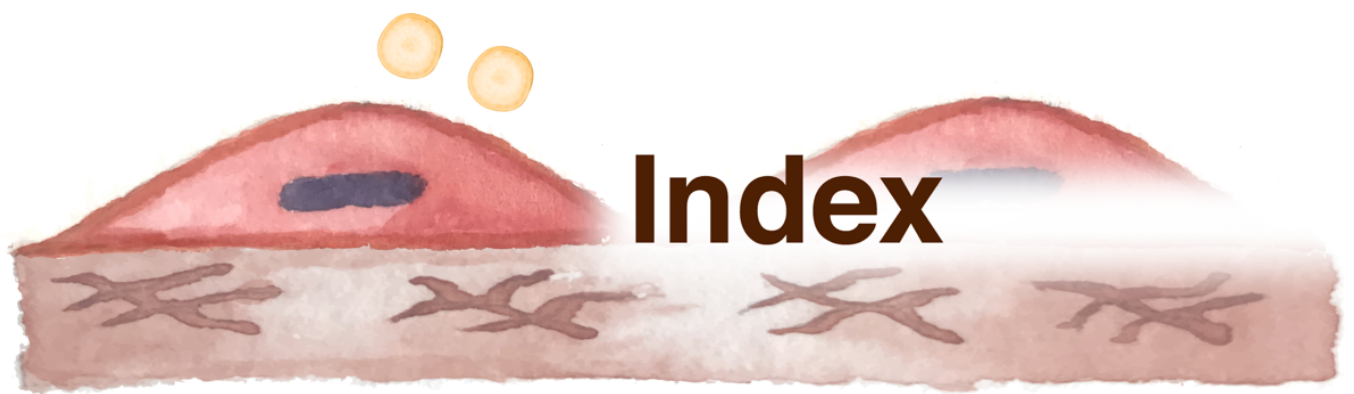




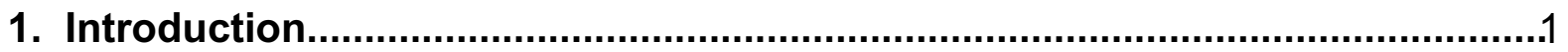

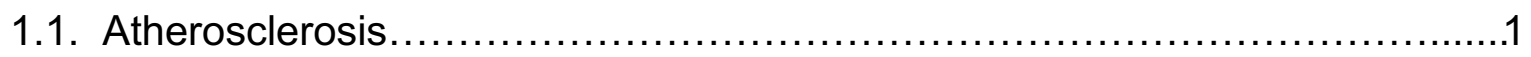

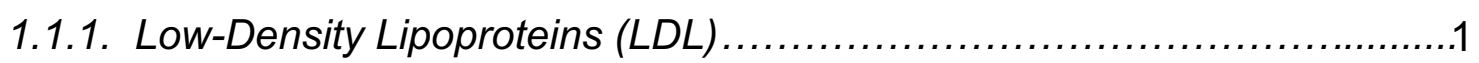

1.1.2. Atherosclerosis-prone sites......................................

1.1.3. $L D L$ entry in the artery wall ............................................ 5

1.1.3.1. Endothelial $L D L$ transcytosis................................... 5

1.1.4. $L D L$ retention in the artery wall...........................................

1.1.5. Response to $L D L$ retention and atherosclerosis initiation .................

1.1.6. Atherosclerosis progression .............................................10

1.1.7. Why does atherosclerosis initially attack atherosclerosis-prone sites? 11

1.1.8. Previous mapping studies of $L D L$ entry and retention rates............... 12

1.2. Animal models of atherosclerosis............................................

1.2.1. Lipid metabolism ......................................................

1.2.2. Mouse models of atherosclerosis................................... 17

1.2.3. Tools to lower $L D L$ levels in mice.................................... 19

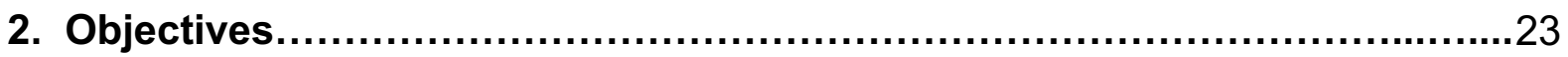

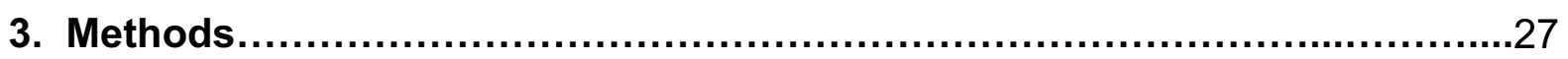

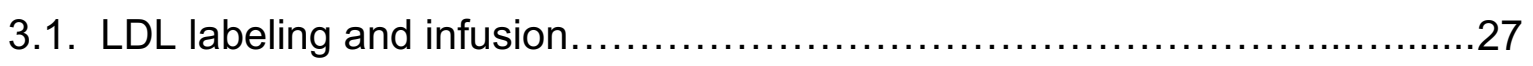

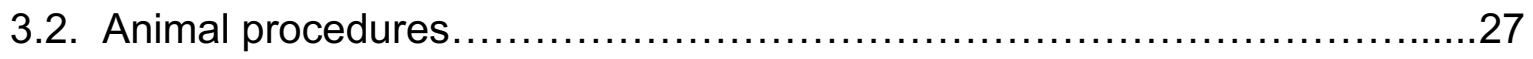

3.2.1. Fluorescently labeled human $L D L$ injections........................28

3.2.2. Tools to control LDL levels..........................................

3.2.3. Endpoint procedure............................................ 31

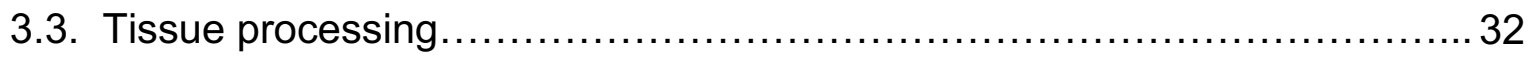

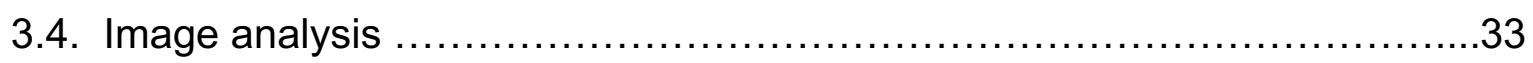

3.4.1. Quantification of $A p o B$ in serial cross sections...........................33

3.4.2. Quantification of amount and localization of labeled LDL in en face

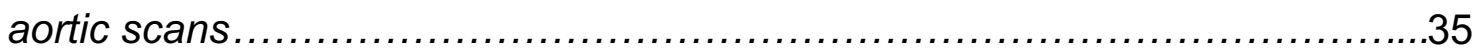

3.4.3. Quantification of amount and localization of labeled LDL and macrophages in whole-mount confocal images of the ascending aorta.

3.4.4. Quantification of $L D L$ retention and macrophage content in sections from ascending atherosclerotic aorta ..................................... 37

3.5. Arterial gene expression profiling by RNA sequencing .........................38

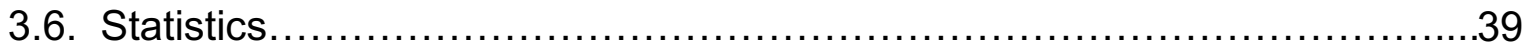




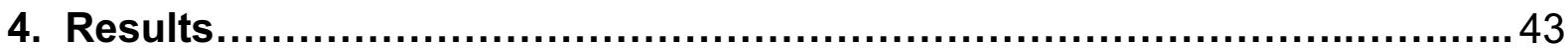

4.1. LDL accumulation precedes plaque development in mice..................43

4.2. Development and validation of high-resolution mapping methods

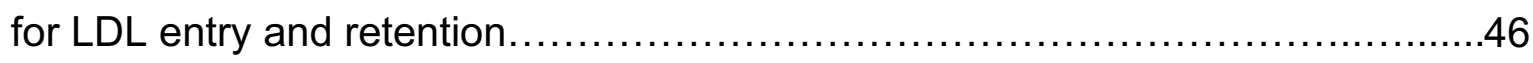

4.3. PCSK9 does not directly affect LDL entry or retention......................49

4.4. LDL retention is rate limiting for LDL accumulation in pre-atherosclerotic arteries.

4.5. Rates of total LDL entry, but not retention, increase during the pre-atherosclerotic phase. .53

4.6. Gene expression changes in pre-atherosclerotic arteries. .55

4.7. Changes in LDL entry and retention are heterogeneous across

the aortic arch. .57

4.8. Saturation of LDL retention is intrinsic to the arterial wall.

4.9. LDL entry and retention rates predict the course of plaque development.

4.10. Atherogenesis is associated with loss of saturation of LDL retention mechanisms

5. Discussion.

5.1. Functional studies reveal the importance of both LDL entry and LDL retention processes.

5.2. Which process determines atherosclerosis susceptibility?.

5.3. Rates of LDL entry and retention define regions with differing atherosclerosis susceptibility.

5.4. The atherosclerosis-intermediate susceptibility region has saturable LDL retention

5.5. The conversion from saturable to non-saturable LDL retention accompanies the onset of atherosclerosis

5.6. Synthesis of findings in a model.

6. Conclusions.

7. References. 


\section{Index Figures}

Figure 1. Atherosclerosis-prone sites and blood flow.. .................................... 3

Figure 2. Steps by which LDL initiate atherogenesis.. ..................................... 4

Figure 3. Structure of proteoglycans and LDL-proteoglycan interaction. .................. 8

Figure 4. Lipid metabolism and transport. ........................................................ 15

Figure 5. PCSK9-mediated LDLR degradation. .......................................... 18

Figure 6. Effect of ApoB ASO treatment. ..................................................... 20

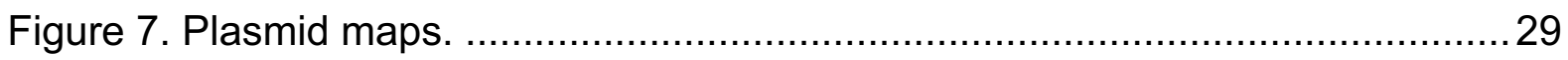

Figure 8. ApoB staining analysis in sections of ascending aorta and aortic root. .....34

Figure 9. Analysis of Atto680-LDL in en face thoracic aorta scans.......................... 35

Figure 10. Co-localization analysis of LDL entry/retention areas and LDL retention in high and low content macrophage areas.

Figure 11. LDL retention and macrophage content analysis in atherosclerotic aortas.

Figure 12. Induction of atherosclerosis through PCSK9 overexpression.................43

Figure 13. LDL accumulation and atherogenesis in mouse ascending aorta.. ........ 44

Figure 14. LDL accumulation and plaque development in mouse aortic root. .......... 45

Figure 15. Method used to measure LDL entry and retention in arteries................46

Figure 16. Localization of labeled-LDL entered and retained in the artery wall........47

Figure 17. LDL entry and retention measurement techniques. ............................48

Figure 18. Linear relationship between fluorescent signal and arterial wall content of

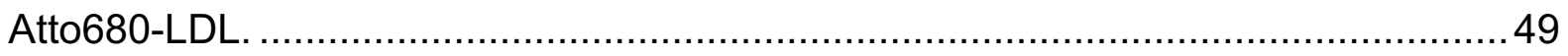

Figure 19. Atto680-LDL entry and retention in Ldlr knockout mice injected with rAAVPCSK9 or rAAV-empty viral vectors.

Figure 20. Experimental design to compare LDL entry and retention in preatherosclerotic and normal arteries

Figure 21. Global LDL retention is reduced despite increased entry into the preatherosclerotic aorta.

Figure 22. Progression of LDL entry and retention during the pre-atherosclerotic phase.

Figure 23. Analysis of gene expression changes in pre-atherosclerotic arteries. ....55

Figure 24. Regulated genes in pre-atherosclerotic arteries. 56 
Figure 25. Topography of LDL entry and retention distribution across the aortic arch in en face scans.

Figure 26. Topography of LDL entry and retention distribution across the aortic arch in whole-mount images.

Figure 27. The border zones of the inner curvature region in pre-atherosclerotic aortas show high rates of LDL entry and retention. 60

Figure 28. Effect of short-term hypercholesterolemia on LDL retention. 61

Figure 29. LDL retention after 3 days of hypercholesterolemia. 62

Figure 30. Effect of rapid hypercholesterolemia reversal on LDL retention. 63

Figure 31. LDL retention after 3 days of LDL lowering. 63

Figure 32. Correlation between the topography of LDL entry and retention and topography of atherosclerotic lesions. 64

Figure 33. LDL entry and retention rates in the pre-atherosclerotic wall predict how atherosclerosis develops 65

Figure 34. Atherogenesis is associated with loss of saturation of LDL retention mechanisms.

Figure 35. Schematic representation of the functional studies showing LDL entry and LDL retention as necessary steps for atherosclerosis initiation.

Figure 36. Model depicting the dynamics of LDL entry and retention in the aortic arch of normal, pre-atherosclerotic, and lesioned arteries. 


\section{Abbreviations}

*I-LDL Radiolabeled lodine-LDL

ABCA1 ATP-binding cassette sub-family A member 1 transporter

ABCG1 ATP-binding cassette sub-family $\mathrm{G}$ member 1 transporter

ADAMTS-5 A disintegrin and metalloproteinase with thrombospondin motifs 5

ALK-1 Activin receptor-like kinase 1

ApoA Apolipoprotein A

ApoB Apolipoprotein B

APOBEC1 ApoB mRNA editing enzyme, catalytic polypeptide 1

ApoC Apolipoprotein C

ApoE Apolipoprotein E

APS Atherosclerosis-Prone Sites

ASO Antisense Oligonucleotide

CAV-1 Caveolin-1

CCA Common Carotid Artery

CS Chondroitin Sulfate

CVD Cardiovascular disease

DAMP Danger-Associated Molecular Pattern

DC Dendritic Cell

DS Dermatan Sulfate

EC Endothelial Cells

ECM Extracellular Matrix

$\mathrm{FH} \quad$ Familial hypercholesterolemia

GAG Glycosaminoglycan

HCAEC Human Coronary Artery Endothelial Cell

$\mathrm{HCL} \quad H y p e r c h o l e s t e r o l e m i a$

HDL High-Density Lipoproteins

HS Heparan Sulfate

ICAM-1 Intercellular adhesion molecule 1

IDL Intermediate-Density Lipoproteins

IPA Ingenuity Pathway Analysis

KS Keratan Sulfate

LDL Low-Density Lipoprotein 
LDLR Low-Density Lipoprotein Receptor

LOX1 Low-Density Lipoprotein Receptor 1

LPL Lipoprotein Lipase

LRP1 LDLR-related protein 1

MAMP Microbe-Associated Molecular Pattern

MCP-1 Monocyte Chemoattractant Protein-1

MTP Microsomal Triglyceride Transfer Protein

NF-kB Nuclear factor-kB

oxLDL Oxidized LDL

PCKS9 Proprotein convertase subtilisin/kexin type 9

PG Proteoglycan

rAAV Recombinant Adeno-Associated Virus

sPLA2 Secretory Phospholipase A2

SR Scavenger Receptors

SR-B1 Scavenger receptor class B type 1

SRA Scavenger Receptor A

SSMase Secretory Sphingomyelinase

TGF- $\beta \quad$ Transforming growth factor beta

VCAM-1 Vascular Cell Adhesion Molecule 1

VLDL Very Low-Density Lipoproteins

VSMC Vascular Smooth Muscle Cell 



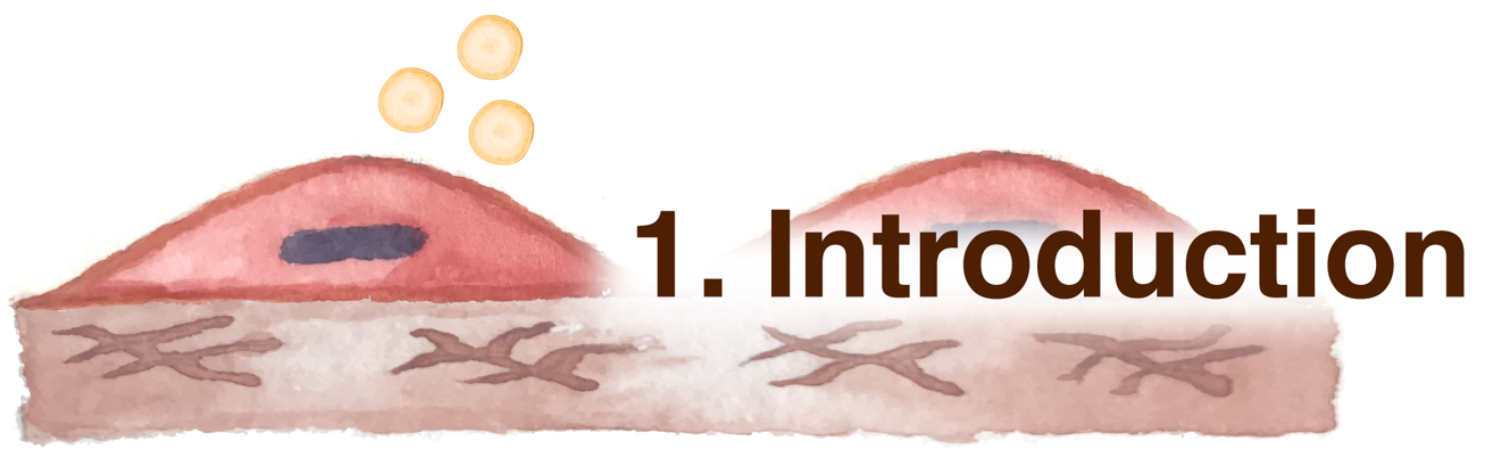




\subsection{Atherosclerosis}

Cardiovascular diseases (CVDs) are the main cause of death worldwide, accounting for 17.9 million deaths per year (31\% of global deaths) (WHO). The majority of CVDs are attributed to atherosclerosis where the development of thrombosis may obstruct blood flow to the heart (coronary heart disease), brain (ischemic stroke), or lower extremities (peripheral vascular disease). ${ }^{1}$ Most people do not know, but atherosclerosis already starts acting during our teenage years. So, during our adolescence, while fighting psychological and social changes, we are unconsciously dealing with an invisible killer that slowly progresses over decades. The atherosclerotic lesions remain innocuous for many years but the longer you live, the higher is the probability of undergoing its clinical complications. For this reason, the increasing average age of the population from the beginning of the $20^{\text {th }}$ century led atherosclerosis to be a central player in the CVDs mortality rates in the world. ${ }^{2}$

Because population growth and the average lifespan are expected to increase further, the identification of preventive measures against the development of atherosclerosis is of great importance.

\subsubsection{Low-Density Lipoproteins (LDL)}

Atherosclerosis is a multifactorial disease initiated and sustained by multiple risk factors, including a high plasma level of low-density lipoproteins (LDLs) and other Apolipoprotein B (ApoB)-containing lipoproteins, arterial hypertension, diabetes mellitus, smoking, and psychosocial factors. ${ }^{3}$ Most individuals that develop clinical symptoms of atherosclerosis have been exposed to several of these risk factors; however, an increased plasma concentration of LDL - compared with the physiological level of 0.5-1 mM present in newborns and other mammals ${ }^{4}$ - represents a particularly important risk factor. Increased LDL is necessary and sometimes sufficient to cause atherosclerosis. None of the other risk factors alone can cause the development of the disease.

Indeed, monogenic hypercholesterolemias, caused by single gene mutations in genes involved in LDL clearance, are associated with high risk of atherosclerosis. ${ }^{5}$ Familial hypercholesterolemia $(\mathrm{FH})$, the most common and most severe form of monogenic hypercholesterolemia, is caused by mutations in the Low-Density Lipoprotein Receptor (LDLR) gene. Patients with FH exhibit very high LDL plasma 
levels irrespective of diet and lifestyle, and $\mathrm{FH}$ homozygotes develop aortic and coronary atherosclerosis already in childhood. ${ }^{6}$ Conversely, life-long reduced levels of LDL, caused by variants in the genes for Proprotein convertase subtilisin/kexin type 9 (PCSK9) or LDLR that increase LDL clearance, is a powerful protectant against atherosclerosis. ${ }^{4}$

In addition, clinical trials showed that LDL lowering treatments (e.g. statins, ezetimibe, or PCSK9 inhibitors) in adult patients with established atherosclerosis significantly reduce atherosclerotic cardiovascular events even in the presence of other risk factors, supporting the crucial role of increased LDL plasma levels not only in atherosclerosis initiation but also in lesion progression. ${ }^{4,7}$

The causal link between blood cholesterol and atherogenesis was unveiled already by Ignatowski and Anitschkow between 1908-1913.8,9 Their pioneering work in cholesterol-fed rabbits that developed atherosclerosis was the birth of the "cholesterol hypothesis" for atherosclerosis and the first of many experimental studies investigating the initiating events in atherosclerosis. Indeed, many researchers have since shown that atherosclerosis can be induced in animals that do not spontaneously develop atherosclerosis (such as rabbits, mice, rats, guinea pigs, hamsters, birds, dogs, and non-human primates) by increasing the level of LDL or other ApoBcontaining lipoproteins via feeding atherogenic diets or genetic modification. ${ }^{10}$ It is also now clear that cholesterol per se is not pathogenic as the increased plasma concentration of other cholesterol transporters, such as high-density lipoproteins (HDL) and erythrocytes, does not cause atherogenesis. ${ }^{11}$

\subsubsection{Atherosclerosis-prone sites}

The artery wall consists of three layers: arterial intima (innermost layer), arterial media (the middle layer) and adventitia (outermost layer). The arterial intima has a single layer of endothelial cells (ECs), in contact with the bloodstream, and a subendothelial layer of connective tissue. The sub-endothelial space comprises scattered vascular smooth muscle cells (VSMCs), resident dendritic cells (DCs), resident macrophages and an extracellular matrix (ECM) that is rich in elastic fibers and proteoglycans. The intima is separated from the arterial media by the internal elastic lamina, composed of fenestrated elastic fibers. The arterial media consists of multiple layers of VSMCs and ECM rich in elastic tissue, collagen, and proteoglycans and it is 
separated from the adventitia by the external elastic lamina. The adventitia consists of connective tissues with collagen, elastic fibers and interspersed fibroblasts, VSMCs, DCs and macrophages. ${ }^{12,13}$

The luminal surface of blood vessels is exposed to hemodynamic shear stress, which is defined as the force that the blood flow exerts on the vessel wall. Straight artery segments, such as the common carotid artery (CCA), are exposed to uniform and unidirectional laminar flow with high shear stress (higher than 12 dyne/cm${ }^{2}$ ). Part of vessel bifurcations and the inside of vascular curvatures are subjected to disturbed blood flow with low shear stress and directional changes $\left( \pm 4\right.$ dyne $\left./ \mathrm{cm}^{2}\right) .{ }^{14}$ In humans, these areas often develop a thickened arterial intima and they are the sites where the first lesions develop and where most culprit lesions causing clinical disease are located. Sites of the arterial tree in which lesions are initiated are defined as atherosclerosis-prone sites (Figure 1).

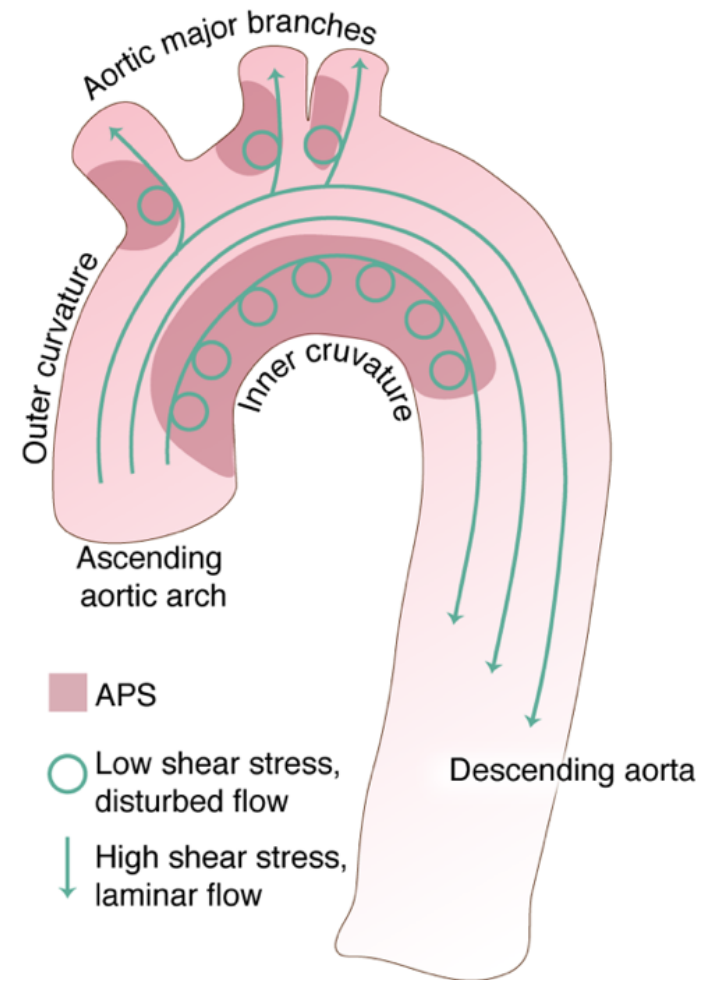

Figure 1. Atherosclerosis-prone sites and blood flow. In the thoracic aorta, the atherosclerosis-prone sites (APS) (dark pink) correspond to branch points and the inner curvature region of the aortic arch, which are subjected to low shear stress because of disturbed blood flow (green circle). The straight segments (outer curvature and descending aorta) are subjected to high shear stress because of unidirectional laminar blood flow (green arrows).

The importance of altered blood flow in lesion development was recognized by Caro et al. already in $1969 .{ }^{15}$ Since then, numerous studies have reported the important connection between disturbed blood flow and the initiation and development of atherosclerosis. ${ }^{16}$ In hypercholesterolemic mice, a causal relationship has been demonstrated by the placement of a mildly constrictive perivascular collar in the 
common carotid artery. ${ }^{17}$ The straight portion of the common carotid artery is normally fully protected from atherosclerosis development, but the disturbed blood flow that results from the insertion of the collar transforms it to an atherosclerosis-prone site with rapid lesion formation. ${ }^{17}$

Why the atherosclerosis-prone sites at branch points and curvatures have a particular susceptibility towards developing atherosclerosis when LDL levels are high is not yet understood and is the overall topic of the present thesis. As described in the following sections, there are multiple steps in the mechanism by which LDL initiate atherogenesis: LDL entry into the artery wall, LDL retention in the sub-endothelial space, LDL modification, and a maladaptive inflammatory response to the accumulated modified LDL (Figure 2). Topographical differences in the activity of these sub-mechanisms are clear candidates for regulating atherosclerosis susceptibility of the arterial tree, but which is the limiting factor remains unsettled.

Lumen

(1) LDL entry

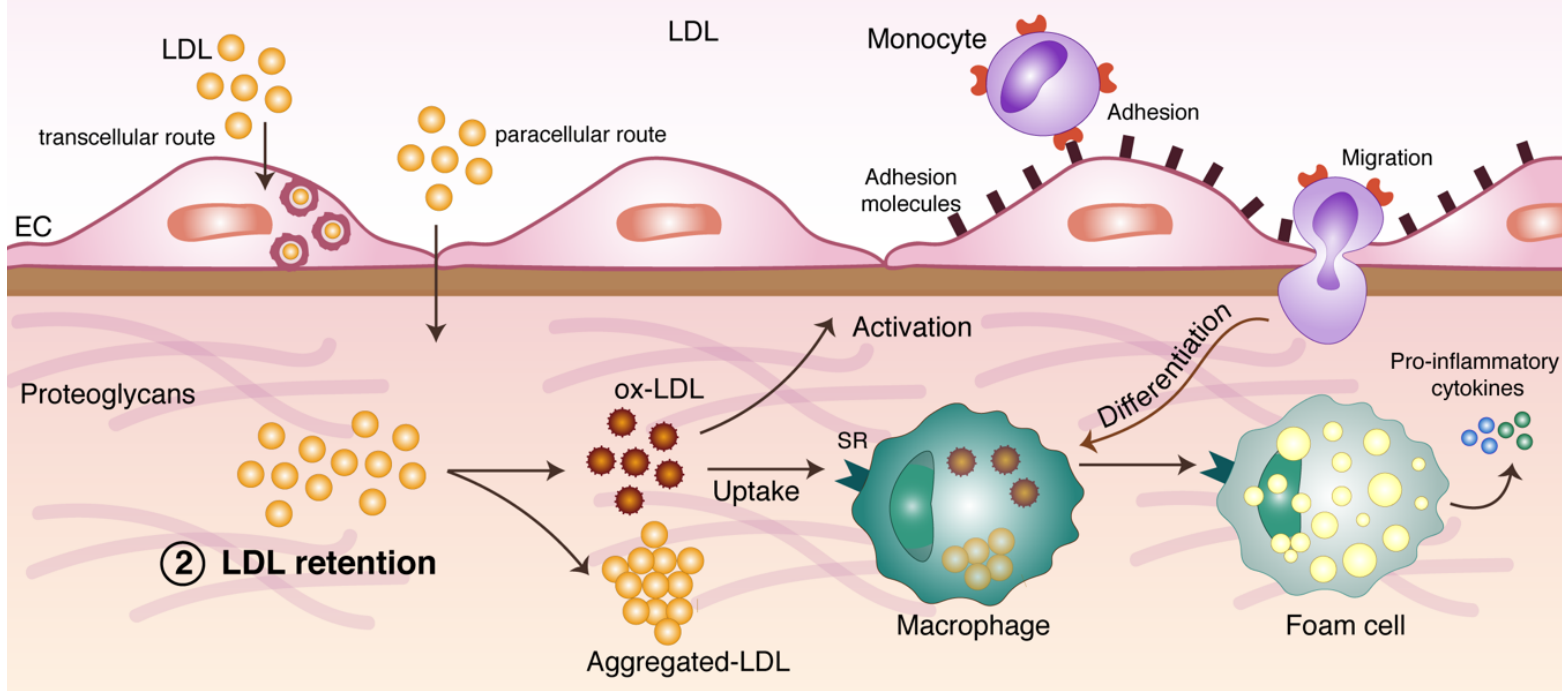

Sub-endothelial space

(3) LDL modification

Figure 2. Steps by which LDL initiate atherogenesis. Plasma LDL cross the endothelium by transcellular route or paracellular route (LDL entry) and they become retained by binding to intimal proteoglycans (LDL retention). Once retained in the sub-endothelial space, LDL are modified through oxidation or aggregation (LDL modification) and modified-LDL act as chronic stimulators of the innate and adaptive immune systems, triggering a maladaptive inflammatory response. $\mathrm{EC}=$ endothelial cell, $\mathrm{SR}=$ Scavenger receptor . 


\subsubsection{LDL entry in the artery wall}

The development of atherosclerosis is triggered by the accumulation of ApoBcontaining lipoproteins, mainly LDL, in the arterial intima. Therefore, LDL must cross the arterial endothelium from the bloodstream and enter the sub-endothelial space. Yet the precise mechanisms underlying this trans-endothelial flux of LDL into the arterial wall has been the subject of debate for decades. ${ }^{18,19}$ LDL can cross the endothelial monolayer by passing between adjacent cells (paracellular route) or by passing through the cytoplasm of individual cells (transcellular route or transcytosis). ${ }^{20}$ Electron microscopic studies favor the latter route, showing the internalization of LDL into cellular vesicles (caveolae), while the transit of LDL through intercellular junctions is not conspicuous. ${ }^{21}$ Also, the size of LDL (over $20 \mathrm{~nm}$ ) would require the loosening of inter-endothelial junctions for the paracellular route to occur;22 yet early atherosclerotic lesions display an intact endothelium. ${ }^{23}$ Some investigators sustain the paracellular route as the main LDL transport route, suggesting that LDL exit the bloodstream at areas of damaged or dividing endothelium. ${ }^{24,25}$ Although the rate of endothelial mitosis is very low (lower than $0.05 \%)^{22}$ and endothelial apoptosis or denudation is not typical in early stages of atherosclerosis, ${ }^{23}$ it may only take a few of such breaches to facilitate intimal LDL accumulation. ${ }^{26}$ In summary, the route by which LDL cross the endothelium is controversial, but current evidence for the early stages of atherosclerosis initiation favor transcytosis. In advanced lesions, other routes could acquire additional importance.

\subsubsection{Endothelial LDL transcytosis}

Transcytosis can be divided into two main types of processes. In direct transcytosis, macromolecules are transported directly from the luminal side to the basolateral side of endothelial cells (i.e. caveolae-mediated transcytosis) facilitated by specific binding to endothelial receptors or via fluid-phase or non-specific binding to membranes. ${ }^{27}$ In indirect transcytosis, macromolecules are endocytosed into early endosomes, transferred to recycling endosomes, and exocytosed on the basolateral side of endothelial cells (i.e. LDLR-mediated transcytosis). ${ }^{27}$

The identification of endothelial receptors involved in arterial LDL transcytosis is of high importance, because their regulation may determine susceptibility to atherosclerosis and be target for therapy. Several lines of evidence suggest that 
transcytosis of LDL across ECs of the systemic circulation is independent from the high affinity LDL receptor, despite its implication in LDL transcytosis across the bloodbrain barrier. ${ }^{28}$ It has been shown that the methylation of $A p o B$, which inhibits its recognition by LDLR, does not affect LDL entry in the arterial wall in rabbits. ${ }^{29}$ Also, PCSK9-mediated LDLR degradation has no effect on LDL transcytosis in primary human coronary artery endothelial cells (HCAECs). ${ }^{30}$ Instead, the Scavenger receptor class B type 1 (SR-B1) and the Activin receptor-like kinase 1 (ALK-1) have both been identified as LDL transcytosis-mediators in ECs of the systemic circulation.

SR-B1, known as a high affinity HDL receptor, has been shown to be able to bind native LDL. ${ }^{31}$ In HCAECs, LDL colocalizes with SR-B1 and its overexpression increases LDL transcytosis, while its knockdown has the opposite effect. ${ }^{30}$ Competition with an excess of HDL also decreases LDL transcytosis ${ }^{30}$. The implication of SR-B1 in LDL transcytosis was also demonstrated by lower intimal accumulation in aortas from SR-B1-deficient mice after ex vivo perfusion with fluorescently-labeled LDL, compared with wild-type mice. ${ }^{30}$ The signaling pathway downstream of SR-B1 is starting to be deciphered and involves the association of the guanine nucleotide exchange factor (GEF) Dedicator of cytokinesis 4 (DOCK4) with the C-terminal cytoplasmic tail of SR-B1, with subsequent activation of GTPase Rac1 and recruitment of its downstream effectors. ${ }^{32}$

ALK-1 is a member of the Transforming growth factor beta (TGF- $\beta$ ) receptor superfamily and was identified as a low-affinity high capacity receptor for LDL. ${ }^{27}$ As SR-B1, ALK-1 colocalizes with LDL and its overexpression increases while knockdown decreases LDL transcytosis. ${ }^{33}$ Moreover, the endothelium-specific knockout of Alk1 in Ldlr knockout mice leads to reduced fluorescently-labeled LDL accumulation in the aortic endothelium. ${ }^{33}$

Both SR-B1 and ALK-1 have been reported to reside in caveolae, ${ }^{34,35}$ small bulb-shaped plasma membrane invaginations of $\sim 50-80 \mathrm{~nm}$ in diameter that are enriched in cholesterol and sphingolipids and able to undergo endocytosis. ${ }^{27}$ Caveolin1 (CAV-1) is the main protein constituent of caveolae, and it has been shown to play a role in atherogenesis. CAV-1 deficiency in Apolipoprotein $\mathrm{E}$ deficient (Apoe $\left.{ }^{-/-}\right)$mice reduces atherosclerotic lesions, while endothelial CAV-1 re-expression promotes lesion expansion. ${ }^{36,37}$ The genetic ablation of CAV-1 results in impaired arterial LDL transcytosis in mice, suggesting caveolae-mediated transcytosis as a major regulator of LDL entry into the vessel wall. ${ }^{38}$ 


\subsubsection{LDL retention in the artery wall}

LDL normally flux into the arterial wall, most likely by transcytosis, and efflux back into the bloodstream. Therefore most LDL particles that enter the artery wall, leave without contributing to the intimal LDL accumulation that finally triggers atherosclerosis. ${ }^{39}$ Indeed, after decades of research there is now a large body of evidence to support the "response-to-retention hypothesis" of atherogenesis. ${ }^{23}$ This hypothesis states that the retention of ApoB-containing lipoproteins, mainly LDL, in the intimal extracellular matrix is a key mechanism in intimal LDL accumulation and atherosclerosis. It builds on pioneering work performed between the 1940s and 1980s, which collectively demonstrated that LDL interact with proteoglycans (PGs) of the artery wall. ${ }^{40-42}$

LDL consist of a lipid core of triacylglycerols and cholesteryl esters, surrounded by a layer of phospholipids, unesterified cholesterol and apolipoproteins. ApoB is the main apolipoprotein of LDL and human LDL contain a single copy of ApoB100 (4536 amino acids). In mice, many LDL instead have the truncated form of ApoB called ApoB48, which is also the form found in intestinally-derived lipoproteins. ${ }^{43}$

Proteoglycans are complex macromolecules that, together with elastin and collagens, constitute the intimal extracellular matrix ${ }^{44}$. Vascular proteoglycans are composed of a core protein and one or more covalently attached glycosaminoglycans (GAGs), linear polysaccharides consisting of repeating disaccharide units of chondroitin sulfate (CS), heparan sulfate (HS), dermatan sulfate (DS) or keratan sulfate (KS). ${ }^{43}$ Proteoglycans are negatively charged due to the sulfate and carboxylic acid groups in their GAG side-chains. The artery wall proteoglycans include versican, perlecan, decorin, biglycan, and syndecan -4.43

During the first steps of atherogenesis, the negatively charged GAG chains of the arterial intimal proteoglycans bind LDL via ionic bonds to positively charged aminoacyl residues on ApoB (Figure 3). These sites have been mapped and are located in site B-lb (residues 84-94) ${ }^{45}$ in ApoB48 and in site B (residues 3359-3369) ${ }^{46}$ and site $A$ (residues 3148-3158) ${ }^{47}$ in ApoB100, the latter only being exposed when LDL is modified by secretory phospholipase A2 (sPLA2). ${ }^{47}$ In addition, ApoB48-LDL typically contain numerous molecules of ApoE, presenting a proteoglycan-binding domain almost identical to site $B$ of ApoB100. ${ }^{11}$ Most proteoglycans have been 
demonstrated to bind to LDL in vitro. ${ }^{46,48}$ However, biglycan and perlecan seem to be the major LDL-binding proteoglycan in human and murine vessels, respectively. ${ }^{42,49}$

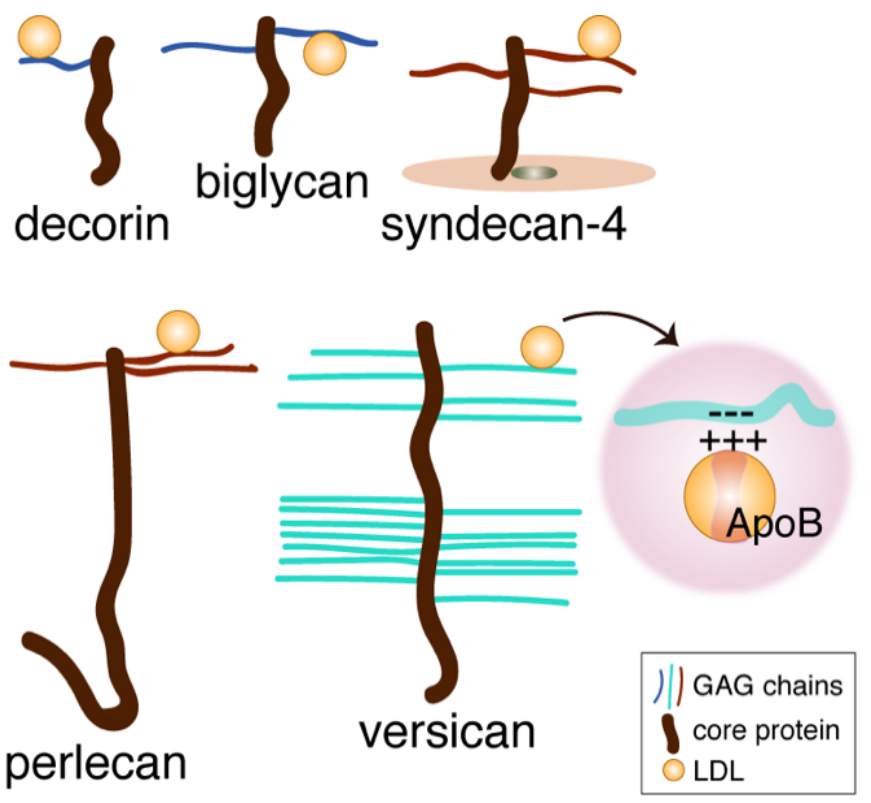

Figure 3. Structure of proteoglycans and LDL-proteoglycan interaction. The negatively charged glycosaminoglycan side chains of the arterial intimal proteoglycans (versican, perlecan, decorin, biglycan, and syndecan-4) bind LDL via ionic interactions to positively charged aminoacyl residues on ApoB.

(Based on Fogelstrand and Borén, 2012) (3 $^{3}$

LDL retention in the sub-endothelial space of the arterial intima seems to be strongly dependent on the intrinsic characteristics of lipoproteins and on the capacity of the vessel wall to bind them. Atherogenicity of LDL particles in terms of affinity for proteoglycans is modulated by their lipid and protein composition. The surface lipid composition $^{50}$ and the lipid core composition ${ }^{47}$ affect the conformation of $A p o B$ and therefore the LDL-proteoglycan interaction. Regarding the protein composition, it has been shown that the Apolipoprotein-C (ApoC)-III levels within LDL alter their proteoglycan-binding affinity. The mechanism is not clear, but an increased ApoC-III content, which itself lacks proteoglycan-binding sites, may induce conformational changes that render $A p o B$ and/or $A p o E$ more accessible to proteoglycans. ${ }^{51}$ Atherogenicity of proteoglycans is modulated by the cellular environment. Elongated GAG chains and GAG sulfation pattern with higher 6:4 position sulfation ratio in the disaccharide units have been associated with increased proteoglycan/LDL binding. ${ }^{52}$ These atherogenic changes in the GAG chains of proteoglycans are influenced by numerous factors including growth factors (i.e. platelet-derived growth factor and transforming growth factor- $\beta$ ), hormones (i.e. angiotensin II), and metabolic factors (i.e. oxidized LDL and free fatty acids). ${ }^{52}$ 


\subsubsection{Response to LDL retention and atherosclerosis initiation}

Once retained in the sub-endothelial space of the arterial intima, LDL may be subjected to multiple enzymatic and non-enzymatic chemical modifications. Free oxygen radicals, hydroperoxides, and lipoxygenases cause oxidization of LDL (oxLDL), and lipolytic enzymes such as lipoprotein lipase (LPL), SPLA2, and secretory sphingomyelinase (sSMase) expose additional proteoglycan-binding sites of ApoB and cause LDL aggregation. ${ }^{47,53,54}$ The LDL-proteoglycan binding per se also induces a physical alteration of LDL with changes in their lipid organization and ApoB configuration that make proteoglycan-bound LDL more susceptible to oxidation and aggregation. ${ }^{55,56}$

The retained and modified LDL acquire molecular patterns shared with microbes (microbe-associated molecular patterns, MAMPs) and damaged tissue (danger-associated molecular patterns, DAMPs) that make them recognizable by the immune response. ${ }^{57}$ Modified-LDL therefore act as chronic stimulators of the innate and adaptive immune systems, triggering a maladaptive inflammatory response. Indeed, they induce in ECs and VSMCs the activation of pro-inflammatory pathways such as the nuclear factor-kB (NF-kB) pathway and the expression of adhesion molecules (i.e. vascular cell adhesion molecule 1 (VCAM-1) and intercellular adhesion molecule 1 (ICAM-1)) and chemoattractants (i.e. monocyte chemoattractant protein-1 (MCP-1)) that stimulate the attachment and transmigration of monocytes into the intimal space. Here, monocytes differentiate into macrophages and dendritic cells. ${ }^{58-}$ 60 These, together with resident VSMCs, engulf modified LDL through scavenger receptors (SR) (i.e. scavenger receptor A (SRA), CD36, SR-B1 and low-density lipoprotein receptor 1 (LOX1)) and possibly other mechanisms, becoming foam cells. ${ }^{61}$ Foam cell formation stimulates the release of pro-atherogenic factors that induce the synthesis of elongated proteoglycans with enhanced affinity for atherogenic lipoproteins, pro-inflammatory cytokines (i.e., interleukin-1 $\beta$ and tumor necrosis factora) and pro-retentive enzymes (LPL, sSMase, and SPLA2) that promote further retention and modification of LDL and aggravation of the vascular inflammation. ${ }^{11}$ Eventually $T$ cells and other inflammatory cells enter the intima and contribute to the inflammatory response. ${ }^{59}$ Accumulated foam cells in several layers give rise to xanthomas that can then develop into progressive atherosclerotic lesions. ${ }^{1}$ 
LDL retention and oxidation may be a self-enhancing process in which retained modified LDL elicit cellular responses that lead to additional LDL entrapment. Ox-LDL stimulate vascular smooth muscle cells to proliferate and secrete proteoglycans with elongated GAG chains presenting increased affinity for LDL. ${ }^{62}$ Furthermore, as the disease progresses, additional LDL retention mechanisms facilize further LDL accumulation. LPL, independently from its enzymatic activity, is able to bind both proteoglycans and LDL, and it therefore acts as a physical bridge in the interaction LDL-proteoglycans enhancing LDL retention in the artery wall. In established plaques, it causes an actual shift in the LDL retention mechanisms from a low-affinity ionic interaction LDL-proteoglycans to a high-affinity bridge interaction LDL-LPLproteoglycans. ${ }^{63}$ Increased expression of LDL aggregating enzymes may further contribute to accelerated LDL accumulation. Aggregated LDL present increased affinity for proteoglycans because of conformational changes that expose additional positively charged domains on $A p o B$ and the presence of multiple GAG-binding sites. ${ }^{64}$ Also, because of their large size, LDL aggregates cannot efflux back into the bloodstream, contributing to additional LDL entrapment. ${ }^{11}$

These shifts in retention mechanisms may explain why lower plasma LDL levels appear to be needed for the growth of plaques than for their initiation; ${ }^{11}$ there is indeed a strong correlation between LDL levels during young adulthood and the risk of developing atherosclerotic cardiovascular events later in life. ${ }^{1}$

\subsubsection{Atherosclerosis progression}

Xanthomas or fatty streaks, corresponding to intimal accumulation of foam cells, are harmless and fully reversible. They can be found already in fetal aortas and very young infants ( $<6$ months of age), especially in the case of hypercholesterolemic mothers. However, their number usually declines during young life to only reappear at atherosclerosis-prone sites during adolescence.65,66 Xanthomas can eventually progress to pathological intimal thickening when foam cells start to undergo apoptosis, causing accumulation of cell debris and extra-cellular lipid pools. These lesions, commonly observed from 20 to 30 years of age at predilection sites, are characterized by a conserved structure of the intima but with accumulated lipid pools beneath the layers of foam cells. ${ }^{67}$ Further accumulation of apoptotic cells, apoptotic remnants and confluence of isolated lipid pools lead to the formation of a 
necrosis area known as necrotic core, characterized by little or no presence of cells and extracellular matrix. This process irreversibly disrupts the normal structure of the intima. Lesions that contain one or more necrotic cores are defined as fibroatheromas. ${ }^{67}$ Fibroatheromas exhibit a characteristic fibrous cap, a layer of VSMCs, and collagenous-proteoglycan matrix located towards the luminal surface of the plaque. Necrotic cores can accumulate calcium deposits until they are completely calcified, giving rise to fibrocalcific plaques. ${ }^{68}$ Advanced plaques can eventually undergo erosion or rupture, causing thrombosis and possibly severe clinical manifestations (coronary heart disease, ischemic stroke, or peripheral vascular disease).

\subsubsection{Why does atherosclerosis initially attack atherosclerosis-prone sites?}

The factors that induce atherosclerosis (LDL, hypertension, etc) are all systemic; yet atherosclerosis affects the arterial tree very heterogeneously attacking mainly low shear-stress regions near branch points and in inner curvatures. Several theories have been proposed to explain this, but the relative importance of each of the processes described below for determining the topography of atherosclerosis is still unsettled.

According to the mass transport theory of atherosclerosis, the arterial uptake of atherogenic material from blood, primarily low-density lipoproteins (LDL), is higher at sites of low or disturbed blood flow. This is explained by a combination of increased residence time of circulating LDL at sites with low wall shear stress and increased endothelial permeability in such areas. ${ }^{69}$ The theory is supported by studies that show increased uptake at atherosclerosis-prone sites of iodinated LDL and Evan's Blue Dye-labeled albumin. ${ }^{70,71}$ The mass transport theory therefore highlights the importance of the rate of LDL entry.

The response-to-retention theory of atherosclerosis states that the retention of LDL to proteoglycans in the intimal layer of the artery wall is the key initiating event in lesion development. Altered shear stress has been shown to induce synthesis of molecules, including proteoglycans, that promote intimal matrix remodeling and therefore lipoprotein binding. ${ }^{23}$ Indeed, the introduction of a disturbed blood flow in the atherosclerosis-resistant common carotid artery is sufficient to increase lipoprotein 
retention. ${ }^{72}$ Also, humans develop spontaneously adaptive intimal thickening at these predilection sites after birth. Adaptive intimal thickening is characterized by vascular smooth muscle cell accumulation within the intima, which represent the main producers of extracellular matrix components. ${ }^{73}$ In contrast to the mass transport theory, the response-to-retention model highlights the importance of retention of the delivered atherogenic material.

The endothelial dysfunction theory of atherosclerosis proposes that low shear stress primes for atherosclerosis by changing endothelial function. Shear stressinduced mechano-transduction mechanisms convert mechanical forces to biochemical responses, activating signal transduction and finally gene and protein expression that determine endothelial cell phenotype. ${ }^{14}$ The diverse endothelial cell phenotype among high and low sheer stress arterial sites is already appreciated at the morphological level. Endothelial cells are fusiform and aligned in the direction of the flow in the presence of high sheer stress while they assume a more polygonal shape and random orientation as the magnitude reduces. ${ }^{74}$ At the functional level, in the straight part of the arterial tree, the athero-protective role of unidirectional blood flow includes an anti-proliferative, anti-apoptotic, anti-oxidant and anti-inflammatory effect on endothelial cells. ${ }^{69}$ In contrast, disturbed blood flow reduces the production of nitric oxide, ${ }^{75}$ increases endothelial turnover, ${ }^{14}$ and activates pro-inflammatory pathways (ie, NF-kB pathway). ${ }^{58}$ Indeed, low-shear stress regions of the normal arterial vasculature exhibit increased expression of chemoattractants and adhesion molecules for monocytes that may facilitate LDL-induced inflammation. The endothelial dysfunction theory therefore highlights differences in the biological response to accumulated LDL.

\subsubsection{Previous mapping studies of LDL entry and retention rates}

A logical way to test the merits of these theories in explaining the differences in atherosclerosis susceptibility is to measure the dynamics of LDL accumulation. The mass transfer theory would predict that atherosclerosis-prone sites correspond to regions with high LDL entry. The response-to-retention theory that atherosclerosisprone sites are those with higher LDL retention. The endothelial dysfunction theory does not presuppose, but also does not exclude, a particular correlation between the topography of LDL deposition and atherosclerosis initiation. 
There is today clear evidence from humans and experimental models that the most atherosclerosis-prone sites are those that accumulate more LDL in the arterial intima when blood LDL levels are high. ${ }^{39,76}$ However, uncertainty remains about whether this is explained by increased entry or retention. ${ }^{11}$ Furthermore, the potential existence of feedback loops already in early LDL accumulation steps, which may facilitate further entry or retention, is incompletely understood.

Previous studies in this field have relied on radioactive labeling to map LDL entry and retention across arteries in animal models, but the results have not been consistent. Schwenke and Carew in 1989 found that atherosclerosis-susceptible arterial regions in rabbits are characterized by higher LDL retention, but not higher LDL entry. ${ }^{39}$ Yet Nielsen et al. subsequently found higher LDL entry in atherosclerosisprone regions in rabbits, ${ }^{70}$ and no association between increased aortic LDL entry and subsequent lesion development was found in pigeons. ${ }^{77}$ Moreover, studies from the 70 s and 80 s in squirrel monkeys and mini-pigs showed higher LDL entry in sites that rarely develop atherosclerosis, such as veins and pulmonary arteries, compared to the atherosclerosis-prone aorta. ${ }^{78,79}$ In mice, the relative contribution of LDL entry or retention to atherosclerosis initiation has not been well studied, and it still remains uncertain whether LDL entry or retention is more characteristic for the atherosclerosisprone sites.

The radioactive techniques used by the majority of previous studies to map LDL entry and retention across arteries are quantitative but have low resolution and typically measure LDL entry and retention rates as averages over large regions. Most of these studies have measured the accumulated radioactivity in the arterial wall after an intravenous injection of radiolabeled iodine-LDL ( $\left.{ }^{*} \mid-L D L\right)$ at different time points. ${ }^{26}$ The assessment of *I-LDL concentration requires either micro-sectioning of the arterial wall prior to the gamma-counting quantification of aortic radioactivity, ${ }^{26,80}$ or histological cross-sectioning of the arterial wall prior to quantitative autoradiography. ${ }^{78}$ Both tissue processing requirements lead to the loss of information on the topographical distribution of LDL entry and retention across the arterial tissue. Alternatively, autoradiography can be done on en face aortas fixed on a radiographic sheet film and apposed on an autoradiography intensifying screen, allowing higher information on the topographical distribution of radiolabeled LDL but with low resolution. ${ }^{81}$ These limitations are a tangible shortcoming because atherogenesis is initially a microscopic phenomenon with sites of incipient atherogenesis and normal 
arterial wall separated by short distances. To understand how rates of LDL entry and retention may determine the susceptibility of the arterial wall to atherosclerosis, further investigation of these processes is needed with techniques that allow higher spatial resolution.

\subsection{Animal models of atherosclerosis}

Measuring the rates of LDL entry and retention in human arteries before and during atherosclerosis initiation is very difficult if not downright impossible. For that reason, previous studies and the present thesis rely on the use of hypercholesterolemic animals to model the onset of atherogenesis.

Many of these types of models have been developed over the last century. In 1908, Ignatowski was the first to induce atherosclerosis in an animal species by feeding rabbits a diet enriched in animal proteins (milk, meat, and eggs). ${ }^{8}$ Since then many other species such as mice, rats, guinea pigs, hamsters, birds, pigs, and nonhuman primates have been used as experimental models of atherosclerosis. ${ }^{10}$ Over the past decades, murine models became the most commonly used to study experimental atherosclerosis because of low cost, rapid reproduction, ease of genetic manipulation and the possibility to evaluate atherogenesis in a relatively short time frame. Although the mouse today is the organism in which the mechanisms of atherosclerosis are best understood, the dynamics of LDL entry and retention have not yet been comprehensibly mapped. The next sections will briefly review the mouse as a model for atherosclerosis and the tools that are available to alter the levels of LDL and other ApoB-containing lipoproteins in them.

\subsubsection{Lipid metabolism}

Mice are "HDL" animals (i.e. HDL is the primary lipoprotein) and they present very low circulating LDL levels. They are therefore relatively resistant to the development of atherosclerosis, even with high-fat feeding. For this reason, mice require genetic manipulation of lipid metabolism genes to develop severe hypercholesterolemia. ${ }^{82,83}$ 
Lipid metabolism relies on plasma lipoproteins that allow transport of dietary lipids from the small intestine to peripheral tissues, the transport of endogenous lipids from the liver to peripheral tissues, and the transport of lipids from peripheral tissues to liver and intestine (reverse cholesterol transport) (Figure 4). ${ }^{84}$ Lipoproteins can be divided according to their density in chylomicrons, chylomicron remnants, very lowdensity lipoproteins (VLDL), intermediate-density lipoproteins (IDL), low-density lipoproteins (LDL) and high-density lipoproteins (HDL).

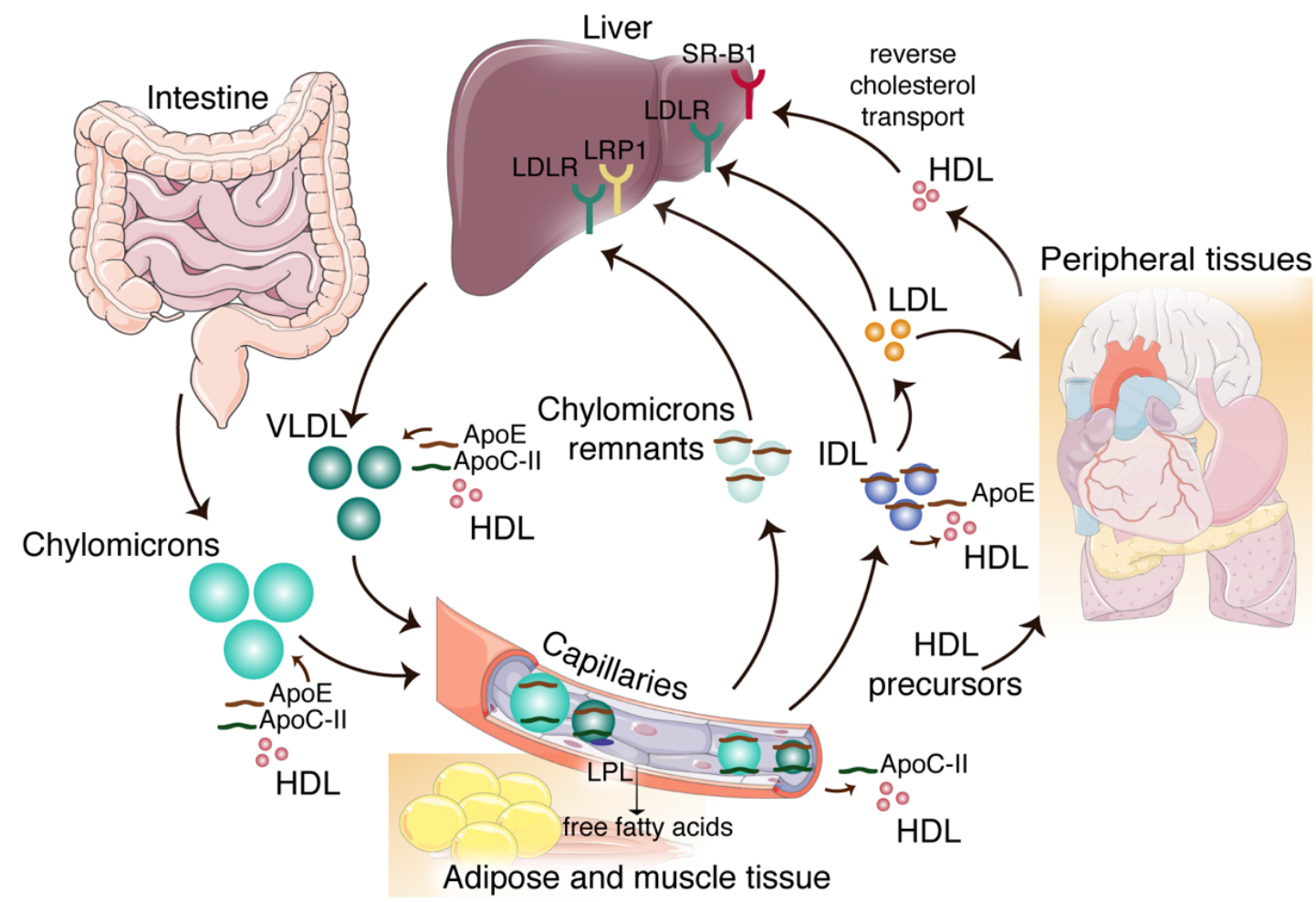

Figure 4. Lipid metabolism and transport. Schematic representation showing the transport of dietary lipids from the small intestine to peripheral tissues, the transport of endogenous lipids from the liver to peripheral tissues, and the transport of lipids from peripheral tissues to liver (reverse cholesterol transport). Details are described in the main text.

Chylomicrons (size, 80-500 nm, density, $<0.95 \mathrm{~g} / \mathrm{ml}$ ) transport dietary triglycerides and cholesterol to peripheral tissues from the small intestine (exogenous pathway). These particles contain the short form of ApoB (ApoB48), created by ApoB mRNA editing enzyme catalytic polypeptide 1 (APOBEC1) editing of the $A p o B$ mRNA, ApoA-I, ApoA-II and ApoA-IV. In the circulation, nascent chylomicrons acquire ApoCII and ApoE from HDL. Lipoprotein lipases (LPL) located in the capillaries of peripheral tissues, mainly adipose and muscle tissues, are activated by ApoC-II and hydrolyze 
triglycerides into fatty acids. As the tissues absorb the fatty acids, chylomicrons shrink to the more pro-atherogenic chylomicron remnants (size, $50 \mathrm{~nm}$; density, $1.006 \mathrm{~g} / \mathrm{ml}$ ), which are cleared from the circulation by both hepatic LDLR and LDLR-related protein 1 (LRP1) through binding to ApoE. ${ }^{84-86}$

VLDLs (size, 30-80 nm; density, 0.95-1.006 $\mathrm{g} / \mathrm{ml}$ ) transport endogenous triglycerides and cholesterol to peripheral tissues from the liver (endogenous pathway). The liver secretes VLDL via exocytosis. Nascent VLDLs contain ApoB100 in humans and acquire ApoC-II and ApoE from HDL once entered into the circulation. In mice, approximately half of the secreted VLDL contain ApoB48 because of some hepatic activity of the APOBEC1 enzyme. ${ }^{87}$ As for chylomicrons, lipoprotein lipases located in the capillaries of peripheral tissues hydrolyze triglycerides into fatty acids. The absorption of fatty acids by the tissues and the transfer of ApoC-II and phospholipids back to HDL convert VLDL to the more pro-atherogenic IDL (size, 25$35 \mathrm{~nm}$; density, 1.006-1-019 g/ml), containing ApoB-100 or ApoB-48 and ApoE. IDL can be cleared from the circulation through LDLR/LRP1 hepatic binding or be further catabolized by lipoprotein lipases, eventually losing ApoE to form LDL (size, 18-28 $\mathrm{nm}$; density, $1.019-1.063 \mathrm{~g} / \mathrm{ml}) .{ }^{85,88}$

In humans, LDL are the main plasma cholesterol carriers that deliver cholesterol to cholesterol-requiring tissues. LDLs that are not taken up in the periphery are predominantly cleared by the hepatic LDLR that binds ApoB100 and internalizes the LDL/LDLR complex by endocytosis into clathrin-coated vesicles. The internalized particles are routed to endosomes, where LDL dissociates from LDLR because of the low $\mathrm{pH}$ in the endosomal lumen. Dissociated LDL is transferred to late endosomes and lysosomes, where the particle is finally digested, and the cholesterol and triglycerides are de-esterified for transport into the cytosol. On the contrary, the LDLR is recycled back to the hepatocyte surface to participate in more rounds of LDL binding and endocytosis. ${ }^{89}$ ApoB-48-LDL in mice lack the LDLR-binding site, however they accommodate a large amount of ApoE that allows clearance through both hepatic receptors, LDLR and LRP1. ${ }^{87}$

HDL (size, 7-12 nm; density 1.063-1.210 $\mathrm{g} / \mathrm{mL}$ ) are involved in reverse cholesterol transport, i.e. the transport of cholesterol excess from peripheral tissues to the liver for metabolism into bile salts. ${ }^{90}$ Intestine and liver synthesize the protein ApoA-I that is secreted in the bloodstream in a lipid-free state. ${ }^{91}$ In peripheral tissues, ATP-binding cassette sub-family A member 1 transporter (ABCA1) mediates the efflux 
of phospholipids and free cholesterol on ApoA-I resulting in the generation of a "nascent" HDL particle. The latter then interacts with SR-B1 and ATP-binding cassette sub-family $G$ member 1 transporter (ABCG1) on peripheral cells to further incorporate cholesterol, forming a mature HDL particle. HDLs interact with SR-B1 in the liver, which allows the transfer of its cholesterol content. ${ }^{92,93}$

\subsubsection{Mouse models of atherosclerosis}

The two most frequently used mouse models of atherosclerosis are the Apoe knockout $\left(A p o e^{-/-}\right)$model and the Ldlr knockout $\left(\right.$Ldlr $\left.^{-1-}\right)$ model.

Apoe $^{-/-}$mice were developed in 1992 and were the first mouse genetic model of atherosclerosis to be created. ${ }^{94}$ ApoE is a ligand for LDLR and LRP1, and the lack of ApoE respectively reduces and totally blocks the clearance of ApoB-containing lipoproteins through these receptors causing massive accumulation of VLDL and chylomicron remnants. ${ }^{95}$ These mice therefore develop atherosclerotic lesions even when fed normal low-fat chow diet. ${ }^{95}$ The severity of the phenotype can be further accentuated by feeding a high fat, high cholesterol Western type diet (WTD) (typically $0.2 \%$ cholesterol, $21 \%$ milkfat). ${ }^{96} \mathrm{Apoe}^{-/-}$mice represent a very useful tool to study atherosclerosis; however plasma cholesterol is mostly carried on chylomicron and VLDL remnants rather than LDL, which is the most frequent pro-atherogenic particle in human atherosclerosis. Also, ApoE has non-lipid-related functions in immune responses and adipose tissue biology, ${ }^{97}$ which may impact atherosclerosis directly.

$\mathrm{LdIr}^{-1-}$ mice were developed in $1993 .{ }^{98}$ In this model, the clearance of lipoproteins through the LDLR is blocked, resulting in moderate accumulations of LDL on normal chow diet and severe hypercholesterolemia on a high-fat diet. ${ }^{98}$ The milder phenotype of the $L d l r^{-1-}$ mouse is unlike humans, where mutations in $L D L R$ yield higher plasma cholesterol levels than mutations in the APOE gene. The milder phenotype of LDLR deficiency in mice is related to their hepatic secretion of ApoB48VLDLs, which is absent in humans. The higher ratios of ApoB48 to ApoB100containing lipoproteins in plasma decrease the relative importance of LDLR-ApoB100mediated versus LDLR/LRP1-ApoE-mediated clearance. ${ }^{87}$ The advantage of the $\mathrm{Ldll}^{-1-}$ model is that LDLR does not appear to exert a multitude of extra-hepatic functions as described for ApoE. ${ }^{99}$ Also, this model has a lipoprotein profile that is more similar to humans with LDL as the main cholesterol-carrying plasma 
lipoprotein. ${ }^{99}$ Furthermore, Ldlr-l- mice mimic the phenotypic events described in human familial hypercholesterolemia caused by homozygous null mutations in the $L D L R$ gene, which is much more common than familial dysbetalipoproteinemia caused by homozygous null mutations in the APOE gene. ${ }^{100}$

Today experimental atherosclerosis research strongly relies on $A p o e^{-/-}$and $L d l r^{-1-}$ models, however, in these mice the hypercholesterolemia phenotype is inborn and the exact time point of initiation of atherosclerosis is therefore not well-defined. More control over the timing of LDL increase can be achieved with virus-mediated gene transfer models, such as the ones obtained though the rAAV-PCSK9 technique.

PCSK9 is a serine protease present in plasma and highly expressed in the liver. ${ }^{101}$ After protein maturation and secretion, circulating PCSK9 binds hepatic LDLR and targets them to lysosomal degradation, diverting the receptor away from the normal recycling to the hepatocyte surface that follows internalization of the LDLR/LDL complex (Figure 5). ${ }^{102}$

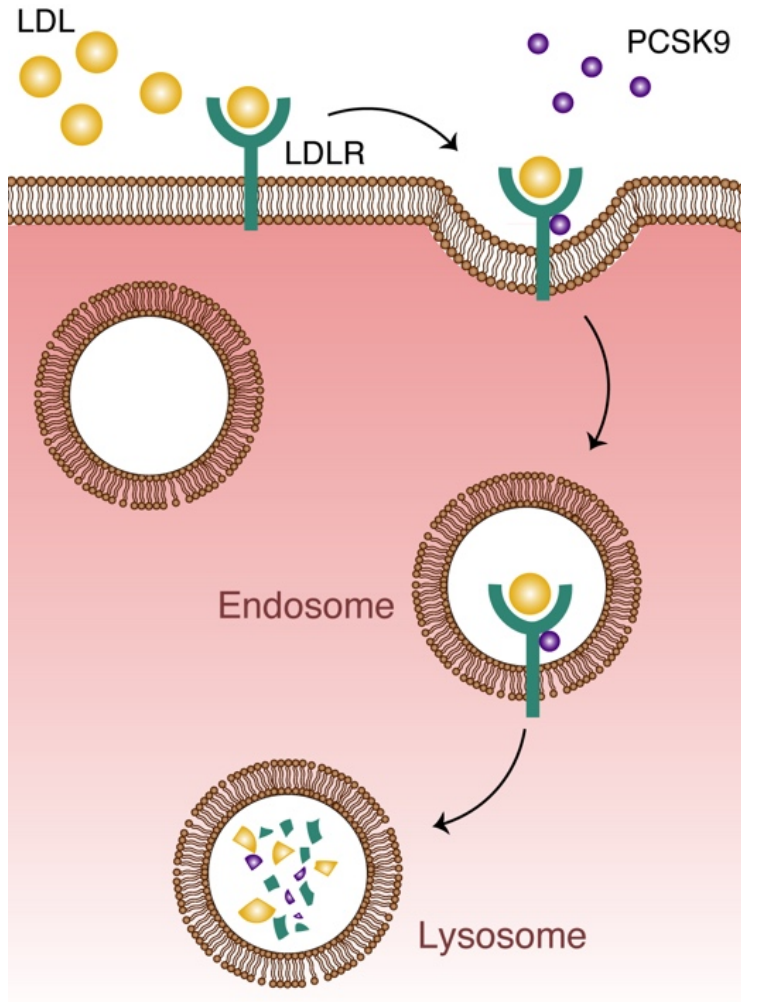

Figure 5. PCSK9-mediated LDLR degradation. Secreted PCSK9 binds to LDLR on the hepatocyte surface and promotes the lysosomal degradation of the PCSK9-LDLR-LDL complex, reducing the total number of LDLR involved in hepatic LDL clearance.

Thus, hyperactivity of PCSK9 reduces the density of LDLR on the surface of hepatocytes, resulting in reduced LDL clearance and raised circulating LDL levels. Clinical studies have shown that PCSK9 gain-of-function mutations are associated with severe familial hypercholesterolemia and premature CVD. ${ }^{103}$ On the other hand, 
loss of function mutations in PCSK9 lead to lower plasma levels of LDL and protection from CVD. ${ }^{104}$ Mice expressing a gain-of-function mutant of PCSK9 delivered by recombinant adeno-associated virus ( $\mathrm{rAAV}$ ) and fed a high fat diet develop sustained hypercholesterolemia and atherosclerosis. ${ }^{105,106}$ Indeed, rAAV-PCSK9-induced mice develop a phenotype similar to that of $\mathrm{Ldlr}^{-/-}$mice. This method presents the advantage of allowing a prompt induction of high LDL levels and atherosclerosis in normal mice at the time point of choice. ${ }^{105}$ This permits more systematic studies of the relationships between the entry, retention and accumulation of LDL, and the onset of atherogenesis than is possible with $\mathrm{Apoe}^{-/-}$and $\mathrm{Ldll}^{-/-}$mice.

Overall, mouse models are powerful tools to improve the understanding of the molecular mechanisms behind atherosclerosis susceptibility, initiation, and progression, but they also present some differences with the human disease. As in humans, lesions in mice start with foam cell formation in regions of disturbed blood flow with predilection sites in the aortic root, the inner curvature of the aortic arch, the brachiocephalic trunk, the abdominal aorta, and the branch points of the left carotid and subclavian arteries. ${ }^{100,107}$ Mice may also develop some atherosclerosis in the proximal parts of the coronary arteries, but this appears to be an extension of aortic root lesions rather than isolated lesions initiated in the coronaries as is generally the case for coronary artery disease in humans. ${ }^{100}$ Also, while intimal thickening with VSMCs accumulation in the intima usually occurs in early lesions in humans, this is not the case in mice that exhibit a very thin intima consisting only of endothelium and scattered dendritic cells. ${ }^{108}$

\subsubsection{Tools to lower LDL levels in mice}

In addition to tools that can increase LDL and cause atherosclerosis, there are several available tools for reducing LDL levels. Many investigators have used them to study the changes in plaque biology that occur when atherosclerotic patients are treated with cholesterol-reducing medications that reduce the incidence of cardiovascular events. ${ }^{109}$ Cholesterol-lowering drugs for use in humans include, among others: statins, $\beta$-Hydroxy $\beta$-methylglutaryl-CoA (HMG-CoA) reductase inhibitors that block the pathway for synthesizing cholesterol in the liver and increase LDLR levels, thereby reducing LDL plasma levels by as much as $60 \% ; 110$ ezetimibe, an intestine cholesterol absorption inhibitor that reduces delivery of cholesterol to the 
liver, thereby reducing LDL plasma levels by $20 \%,{ }^{110}$ and PCSK9 inhibitors, monoclonal antibodies that lower LDL plasma levels by $50-60 \%$ by binding PCSK9, which in turn decreases the degradation of LDL receptors. ${ }^{110}$

For studies in mice, alternative tools are available that allow correction of the severe hypercholesterolemia of atherosclerosis models. ${ }^{111}$ An indirect model was developed in $2001^{112}$ in which atherosclerotic aortas are transplanted from donor mice with high plasma LDL levels into healthy recipient mice with low plasma LDL levels. ${ }^{112}$ Other important models are based on the genetic deletion ${ }^{113}$ or pharmacological inhibition $^{114}$ of the microsomal triglyceride transfer protein (MTP), required for the proper packaging of VLDL and chylomicrons in intestine and liver. Both tools induce rapid reduction in chylomicrons remnants and LDL levels. ${ }^{113,114}$ Furthermore, normalization of plasma cholesterol levels has been achieved through adenovirusmediated re-introduction of the Apoe and $\mathrm{Ldlr}$ genes in $\mathrm{Apoe}^{-/-}$and $\mathrm{Ldlr}^{-/-}$mice, respectively, combined with switching to low-fat chow diet. ${ }^{115,116}$ Finally, in recent years, the use of antisense oligonucleotides (ASOs) against the $A p o B$ mRNA has been implemented as a very convenient and efficient tool to reduce LDL plasma levels. Weekly injections of antisense oligonucleotides targeting Apolipoprotein $\mathrm{B}$ ( $A$ poB ASO) can reduce hepatic $A p o B$ mRNA expression by $90 \%$ and consequently lower VLDL liver secretion and LDL circulating levels (Figure 6). ${ }^{117,118}$

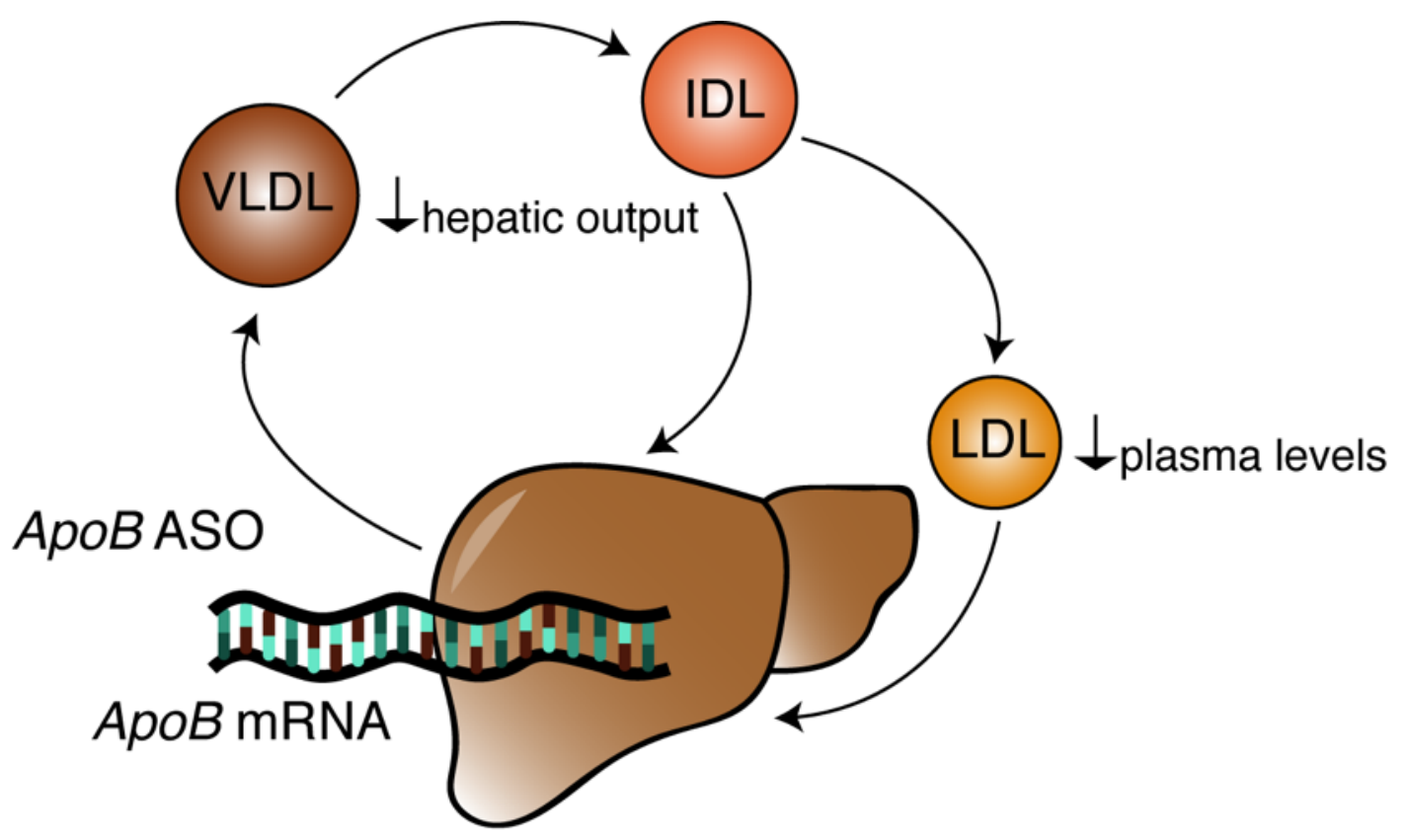

Figure 6. Effect of ApoB ASO treatment. The $A p o B$ ASO targets the hepatic $A p o B$ mRNA thereby reducing the hepatic output of VLDL, which in turn causes a drastic reduction in LDL plasma levels. 



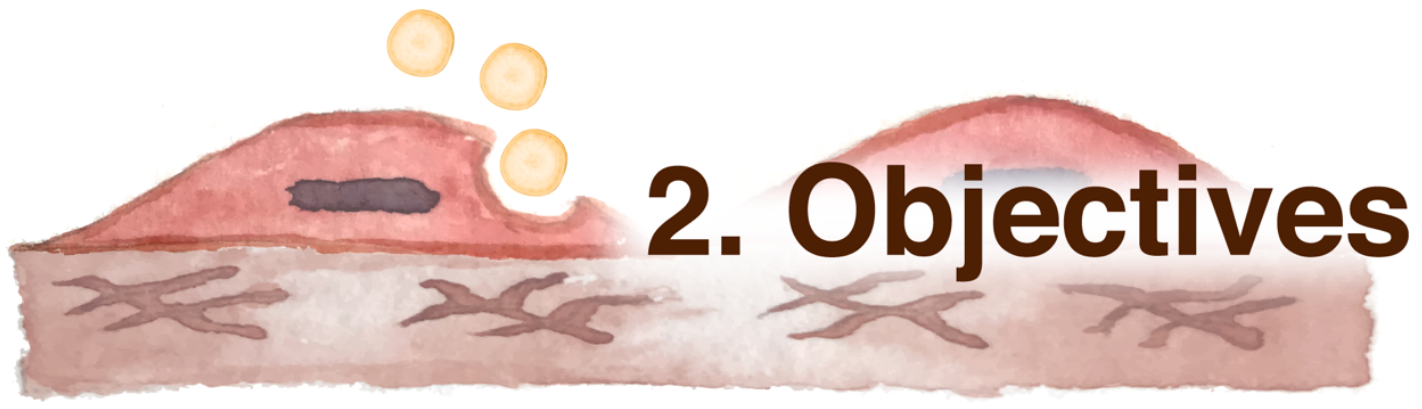


The processes controlling LDL accumulation and atherosclerosis onset at atherosclerosis-prone sites of the vasculature remain incompletely understood. We hypothesized that the use of high-resolution LDL tracking techniques in mice with control of LDL levels would provide new insights into the processes that drive LDL accumulation at some sites while protecting against it at other sites.

To explore this idea, we set the following objectives:

1. To study the temporal relationship between LDL accumulation and onset of atherogenesis in the mouse aorta.

2. To develop techniques that allow mapping of the rates of LDL entry and retention at microscopic resolution in the mouse aortic arch.

3. To evaluate whether rates of LDL entry and/or retention are changed in preatherosclerotic arteries compared with normal arteries and how they vary during the first weeks after the onset of hypercholesterolemia.

4. To analyze the topographical distribution of LDL entry and retention across the mouse aortic arch in normal and pre-atherosclerotic arteries and how this correlates with the sites of atherosclerosis initiation.

5. To assess whether LDL retention rates change in lesioned arteries compared with pre-atherosclerotic arteries. 




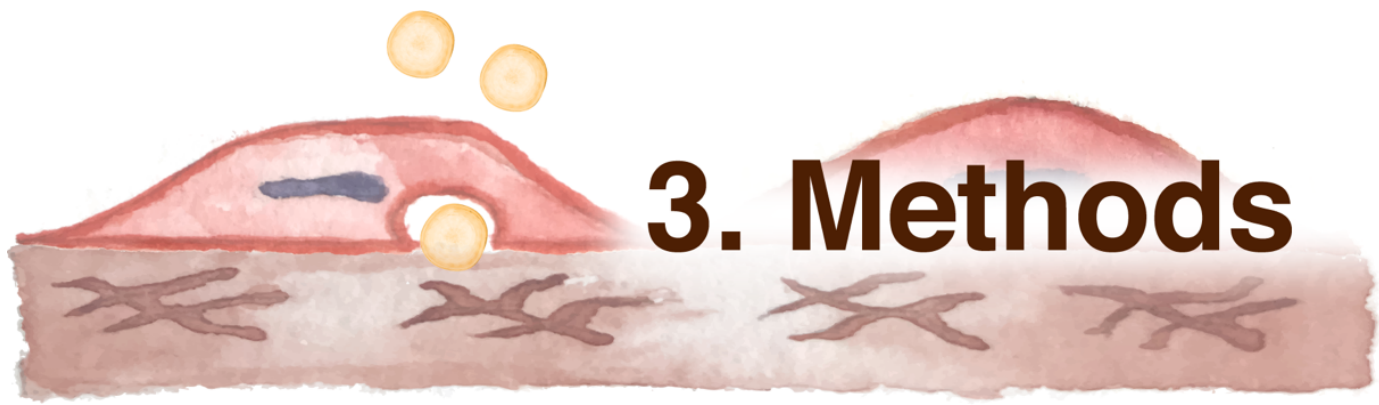




\subsection{LDL labeling and infusion}

Blood for LDL isolation was collected in EDTA-coated tubes from healthy volunteers after informed consent, as approved by the Comité de Ética de la Investigación del Instituto de Salud Carlos III (CEI PI 12_2016-v2). Plasma was isolated by centrifugation and mixed with $\mathrm{KBr}$ to a density of $1.21 \mathrm{~g} / \mathrm{l}$ and layered in a $\mathrm{KBr}$ density gradient column (density layers: 1.006, 1.019, 1.063, and $1.21 \mathrm{~g} / \mathrm{ml}$; Tube, Thinwall Ultra Clear, Beckman Coulter). The column was ultracentrifuged at 40,000 rpm (Beckman Coulter Optima L- 100K Ultracentrifuge, SW 40 Ti Rotor) and $4^{\circ} \mathrm{C}$ for 20 hours. Human LDL was collected from the $1.063 \mathrm{~g} / \mathrm{ml}$ density layer and concentrated using the Amicon system (Amicon Ultra-15, membrane PLTK Ultracel$\mathrm{PL}, 30 \mathrm{kDa}$ ) at 4,000 rpm (Eppendorf Centrifuge 5810, A-4-81 Rotor) and $4^{\circ} \mathrm{C}$ until volume reduction to approximately $2.5 \mathrm{~mL}$. LDLs were then purified on a PD10 desalting column (GE Healthcare) with PBS and quantified using the BCA protein assay kit (ThermoFisher). For en face experiments, the human LDLs were conjugated at a 1:2 molar ratio to Atto680 N-hydroxysuccinimide (NHS) ester fluorochrome (Sigma Aldrich) at $\mathrm{pH} 8.3$, with $0.1 \mathrm{M} \mathrm{NaHCO}$ following the manufacturer's protocol; for confocal microscopy experiments, conjugation was to either Atto565 or $647 \mathrm{NHS}$ ester fluorochrome (Sigma Aldrich). The Atto NHS labels attach to free amines on the protein moiety of LDL, Apolipoprotein B. Human labeled LDLs were then purified on a PD10 column with PBS to remove excess fluorochrome and quantified again using the BCA protein assay kit (ThermoFisher).

\subsection{Animal procedures}

Animal experiments were approved by the ethical review boards at CNIC and Universidad Autónoma and approved by the Comunidad de Madrid (PROEX 266/16). The mice used in this study were male LdIr (Low-density lipoprotein receptor) knockout mice (B6.129S7-Ldlr ${ }^{\text {tm }}$ 1Her/J, Jackson Laboratory) and male wild-type B6 mice (C57BL/6J, Jackson Laboratory). All compared mice were littermates, housed together, and subjected to the same procedures, except for the experimental intervention. 


\subsubsection{Fluorescently labeled human LDL injections}

To measure rates of LDL entry and retention, mice were infused through the tail vein with fluorescently labeled human LDL followed by sacrifice after 1 hour (to measure rate of LDL entry) or 18 hours (to measure rate of LDL retention). In singlelabel experiments, i.e. when evaluating LDL entry and LDL retention between different samples, mice were injected either at 18 hours or 1 hour before the endpoint with 500 $\mu \mathrm{g}$ of human LDL conjugated with either Atto680, Atto647 or Atto565 diluted in PBS. In double-label experiments, i.e. when evaluating the pattern of LDL entry and LDL retention in the same sample, mice were injected at 18 hours and 1 hour before the endpoint with $250 \mu \mathrm{g}$ of human LDL conjugated with different fluorophores (Atto647 or Atto565).

To evaluate the proportionality between the Atto680-LDL infrared signal detected in arteries and the amount of labeled LDL injected, mice were injected with unlabeled LDL alone (500 $\mu \mathrm{g}$ of unlabeled LDL), a mix of unlabeled and labeled LDL with increasing percentages of labeled LDL $(375 \mu \mathrm{g}$ unlabeled LDL $+125 \mu \mathrm{g}$ labeled LDL; $250 \mu \mathrm{g}$ unlabeled LDL+ $250 \mu \mathrm{g}$ labeled LDL; $125 \mu \mathrm{g}$ unlabeled LDL+ $375 \mu \mathrm{g}$ labeled LDL) or labeled LDL alone (500 $\mu \mathrm{g}$ of labeled LDL). Plasma concentrations of infused labeled LDL were determined in blood samples using a FluoriskanAscent fluorometer (Thermo Labsystems).

\subsubsection{Tools to control LDL levels}

LDL levels were increased using a single tail vein injection of recombinant adeno-associated virus encoding a D377Y gain-of-function mutant of PCSK9 (rAAVPCSK9). rAAV-PCSK9-mediated gene transfer leads to high hepatic expression of D377Y-PCSK9 resulting in persistent downregulation of LDL receptors as previously described. ${ }^{105}$ Control rAAV8 virus particles without the PCSK9 encoding cassette were used as control (rAAV-control). The pAAV/D377Y-mPCSK9 plasmid used to create rAAV-PCSK9 particles is composed of an ampicillin resistance cassette, left and right inverted terminal repeats (ITR), a liver-specific promoter (ApoEHCR-hAAT) consisting of the hepatocyte control region (HCR) from the APOE gene and the human a1antitrypsin promoter (hAAT) promoter, the D377Y-mPCSK9 cassette encoding gainof-function form of murine PCSK9, and a bovine growth hormone polyadenylation 
signal (BGHpA). The pAAV/control plasmid used to create rAAV-control particles has the same structure but lacks the D377Y-mPCSK9 cassette (Figure 7). ${ }^{105}$

A

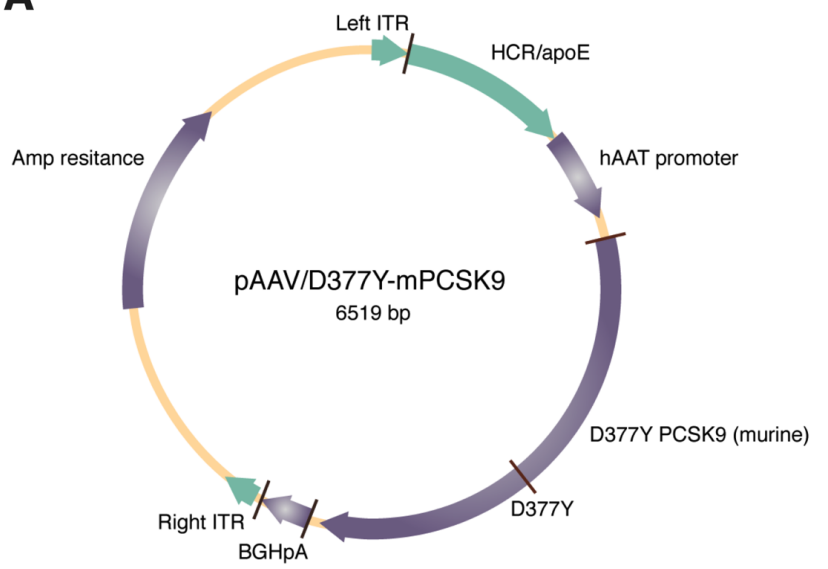

B

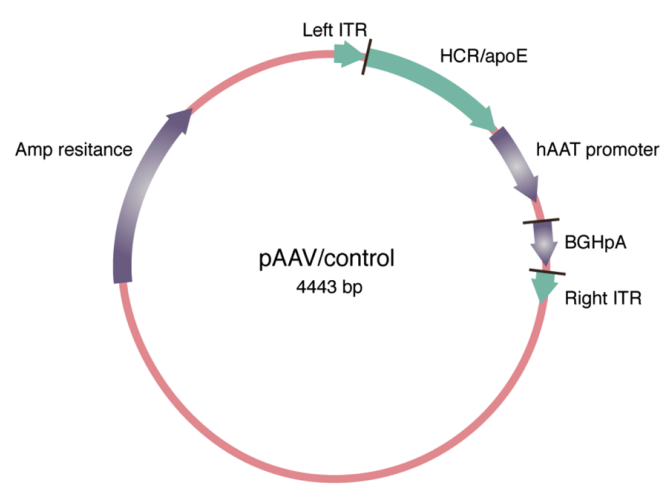

Figure 7. Plasmid maps. A, pAAV/D377Y-mPCSK9 (6519 bp) and B, pAAV/control (4443 bp).

Following transformation and amplification in $\mathrm{Ca}^{+2}$ competent $\mathrm{E}$. coli $\mathrm{H} 5$ bacteria, pAAV-D377Y-mPCSK9 and pAAV-control were purified using the QIAGEN Maxi Plasmid Purification Kit, following manufacturer's instructions. After digestion with the restriction enzymes Ahdl and Bgll to verify the integrity of the terminal inverted repeats (ITR), the viral vector particles were produced by the CNIC viral vector core facility. For the tail vein injection, $1 \times 10^{11}$ viral particles were diluted in $200 \mu \mathrm{l}$ of $\mathrm{NaCl}$ and injected in mice previously kept in a warming chamber to induce vasodilation. Approximately 6 hours after injection, mice were put on high-cholesterol diet (S9167E011, Sniff) or kept on standard laboratory chow diet, according to the aim of the individual experiments. Blood samples for LDL cholesterol and other measures were drawn before viral vector injection and at different time points following injection, depending on the experiment.

LDL levels were decreased using the 14-mer locked nucleic acid (LNA) antisense oligonucleotide targeting $A p o B$ mRNA (ApoB ASO, 5'-3': $A G^{m}$ Cattggtat $T^{m} C A$, where the uppercase letters indicate LNA monomers, lowercase letters indicate DNA monomers, and ${ }^{\mathrm{m}} \mathrm{C}$ stands for LNA-5-methylcytidine) (Exiqon). ${ }^{119}$ The ASO was administered either weekly $(5 \mathrm{mg} / \mathrm{kg})$ or in a single high dose $(10 \mathrm{mg} / \mathrm{kg})$, according to the aim of the individual experiments. The same dose of a standard 15mer LNA negative control antisense oligonucleotide without homology to any known coding or non-coding mouse RNA sequence was used as control (Ctrl ASO, 5'-3': 
aacacgtctatacgc, the position of the LNAs is proprietary information not revealed by Exiqon).

Using the tools to increase and/or decrease plasma LDL levels, the following types of experiments were done (group sizes and further details are discussed in the results section):

- To evaluate the progression in LDL accumulation after the onset of hypercholesterolemia, mice were injected with rAAV-PCSK9 (8 weeks of age) and subsequently kept on high-cholesterol diet for 2, 4, 6, or 12 weeks.

- To rule out a direct effect of PCSK9 on LDL entry and retention in arteries, Ldlr $^{-/-}$mice (8 weeks of age) fed standard laboratory chow diet received a tail vein injection of rAAV-PCSK9 or rAAV-control virus particles and were sacrificed after 3.5 weeks.

- To create groups of mice that differ in plasma LDL levels without differences in hepatic LDL clearance, all mice (8 weeks of age) received a tail vein injection of rAAV-PCSK9 to reduce hepatic LDL clearance and were fed high-cholesterol diet for 3.5w. Some mice (normocholesterolemic group with normal arteries) were concomitantly treated weekly with a dose of $5 \mathrm{mg} / \mathrm{kg}$ of Apob ASO to keep LDL at normal levels (by reducing hepatic VLDL output), while others (hypercholesterolemic group with pre-atherosclerotic arteries) received the same dose of Ctrl ASO. The two groups were compared for rates of entry and retention of labeled LDL and for arterial gene expression.

- To measure LDL entry and retention rates during the first weeks after the onset of hypercholesterolemia, mice received a tail vein injection of rAAVPCSK9 and were started on high-cholesterol diet at 8,10 , or 11 weeks of age. After 4, 2, or 1 weeks of hypercholesterolemia, respectively, rates of LDL entry and retention were measured. This design allowed the use of the same labeled LDL preparation for all groups enabling comparison without potential bias caused by differences in LDL preparation or labeling.

- To assess the rate of labeled LDL retention shortly after the induction of hypercholesterolemia, two groups of mice ( 8 weeks of age) received a tail vein injection of rAAV-PCSK9 and were started on high-cholesterol diet. One group of mice also received a single $5 \mathrm{mg} / \mathrm{kg}$ dose of $A p o B$ ASO while the 
other group received a similar dose of Ctrl ASO. Mice were sacrificed after 3 days.

- To evaluate the effect of rapid LDL lowering on the rate of labeled LDL retention, two groups of mice (8 weeks of age) received a tail vein injection of rAAV-PCSK9 and were kept on high-cholesterol diet for 3.5w. At 3 days before the endpoint, the groups received a single $10 \mathrm{mg} / \mathrm{kg}$ dose of either ApoB ASO or Ctrl ASO.

- To analyze the topography of atherosclerotic lesions at different time points after the induction of hypercholesterolemia, mice (8 weeks of age) received a tail vein injection of rAAV-PCSK9 and were kept on high-cholesterol diet for $4 w, 6 w, 12 w$, and $24 w$.

- $\quad$ To study the rate of labeled LDL retention in atherosclerotic arteries, mice (8 weeks of age) received a tail vein injection of rAAV-PCSK9 and were kept on high-cholesterol diet for $12 \mathrm{w}$. At 1 week before the endpoint, one group of mice received a single $10 \mathrm{mg} / \mathrm{kg}$ dose of $A p o B$ ASO to abruptly lower LDL levels, while the others received a similar dose of Ctrl ASO.

\subsubsection{Endpoint procedure}

Mice were sacrificed by intraperitoneal injection of pentobarbital $(250 \mathrm{mg} / \mathrm{kg})$ and lidocaine $(20 \mathrm{mg} / \mathrm{kg})$. After sacrifice, mice were exsanguinated and perfused first with $\mathrm{KCl}$ solution (50 mM, 30 seconds) and then with $4 \%$ phosphate-buffered formaldehyde (5 minutes) at approximately $100 \mathrm{mmHg}$ through the left ventricle, using the cut right atrium as the drainage route. Mice were then immersion-fixed in $4 \%$ phosphate-buffered formaldehyde for a further 18 hours, followed by storage in PBS at $4^{\circ} \mathrm{C}$ until extraction of the aorta and heart.

Only in the case of sample preparation for RNA sequencing of the inner curvature region of the aortic arch, a different procedure was followed. Mice were euthanized as detailed above but perfused with ice-cold PBS (5 minutes), and aorta extraction and separation of the inner aortic curvature region were performed immediately. Inner aortic curvature samples were then quickly snap-frozen in liquid nitrogen until further processing. 


\subsection{Tissue processing}

For the analysis of cross-sections of the ascending aorta and aortic root, the aorta and heart were extracted from perfusion-fixed mice and the aortic arch was separated from the heart. Both tissues were cryoprotected (24 hours in $25 \%$ sucrose $\mathrm{w} / \mathrm{w}$ followed by 24 hours in $50 \% \mathrm{w} / \mathrm{w}$ sucrose in phosphate-buffered saline [PBS]). Tissues were then embedded in Tissue-Tek O.C.T. Compound (Sakura) and snapfrozen in liquid nitrogen. The ascending aorta was sectioned $(5 \mu \mathrm{m})$ from the proximal end until the branch point of the brachiocephalic trunk. Cross-sections of the aortic root $(5 \mu \mathrm{m})$ were obtained from the commissures of the aortic cusps upwards.

In mice not injected with labeled human LDL, LDL accumulation was quantified by staining for $A p o B$ in the ascending aorta and aortic root sections (at three positions for each mouse, separated by $200 \mu \mathrm{m}$ and $100 \mu \mathrm{m}$ in the two locations, respectively). After antigen retrieval in $0.05 \%$ trypsin solution, sections were stained with goat polyclonal anti-ApoB antibody (dilution 1:300, ab98132, Abcam, UK), which recognizes mouse $A p o B$, followed by HRP-coupled secondary antibody (dilution 1:500, A15999, ThermoFisher) and DAB staining.

In mice injected with labeled human LDL, labeled LDL was detected in ascending aorta sections by its fluorescence and macrophages by staining with rat Anti-Mac-2 (Galectin-3) antibody (dilution 1:100, CL8942AP, CEDARLANE) followed by Alexa Fluor 568 Goat Anti-Rat IgG $(\mathrm{H}+\mathrm{L})$ (dilution 1:500, A-11077, Invitrogen). Three levels for each mouse, separated by $200 \mu \mathrm{m}$, were analyzed by confocal microscopy (Zeiss LSM780 confocal microscope or Leica SP8 Lightning-Navigator confocal microscope). Specificity was evaluated by observing the expected localization pattern and with negative controls without labeled LDL injection or omitting the anti-Mac-2 primary antibody.

For en face imaging scans of aortas from Atto680-LDL-injected mice, extracted aortas from perfusion-fixed mice were opened longitudinally along the outer and inner curvature and along the front of the descending aorta and mounted on microscope slides using SlowFade ${ }^{\mathrm{TM}}$ Gold Antifade Mountant (S36937, ThermoFisher) and coverslips. Microscope slides were scanned using the $700 \mathrm{~nm}$ channel of a LiCor Odyssey Infrared Imaging System. Aortas from mice not infused with labeled human LDL were used as a control for signal specificity. 
For whole-mount confocal microscopy of ascending aortic arches from mice injected with both Atto647-LDL and Atto565-LDL, aortas were extracted from perfusion-fixed mice, and the ascending aortic arch until the brachiocephalic trunk was separated from the rest of the vessel. The ascending aortic arch was opened up to the brachiocephalic trunk along the ventral wall to maintain the atherosclerosis-prone inner curvature region intact. Samples were mounted on microscope slides in SlowFade ${ }^{\mathrm{TM}}$ Gold Antifade Mountant, and images of green autofluorescence, Atto565, and Atto647 fluorescence were acquired with a Zeiss LSM 780 confocal microscope or Leica SP8 Lightning-Navigator confocal microscope. For detection of macrophages in whole-mount preparations of ascending aorta, the specimens were first permeabilized/blocked with PBS containing 0.5\% Triton X-100 and 20\% chicken serum and then further blocked with PBS containing $0.3 \%$ Triton $X-100,5 \%$ bovine serum albumin, and $5 \%$ chicken serum. Aortas were then stained overnight with rat anti-Mac2 antibody (dilution 1:100, CL8942AP, CEDARLANE), followed by Alexa Fluor 488 Chicken anti-Rat IgG $(\mathrm{H}+\mathrm{L})$ (dilution 1:100, A-21470, ThermoFisher). Mac2 staining was performed as a post-staining in specimens already analyzed for Atto647LDL and Atto565-LDL presence.

For the assessment of the topography of atherosclerotic lesions across the ascending aortic arch, aortas were extracted from previously fixed mice and opened as described above. En face images were acquired using a Nikon stereomicroscope.

\subsection{Image analysis}

All quantitative image analysis was performed in Fiji (Fiji is just ImageJ) ${ }^{120,121}$ version 2.0.0-rc-69/1.53c and associated plugins ${ }^{122,123}$ with the contribution of the microscopy unit of CNIC.

\subsubsection{Quantification of ApoB in serial cross sections}

The analysis of DAB signal in color images of ascending aorta and aortic root sections stained for ApoB was performed using the Colour Deconvolution Plugin with the provided "H DAB" vector. ${ }^{122}$ After color separation, regions of interest (ROIs) corresponding to total tissue and plaque were defined manually, and areas were quantified. DAB signal was then detected by pixel thresholding segmentation and the 
area of DAB signal within the total tissue ROI was measured (Figure 8). In the aortic root, the signal in the three coronary sinuses (left, right, and non-coronary sinus) were quantified separately.

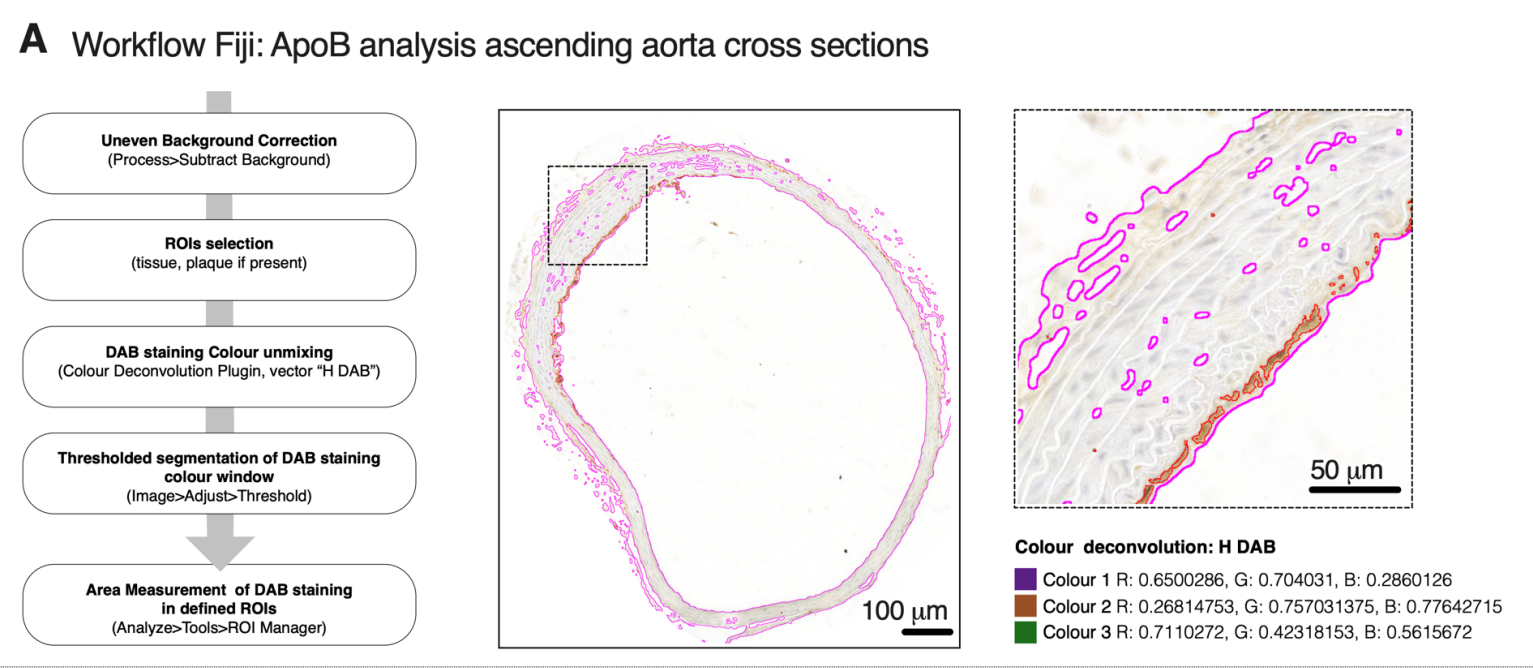

B Workflow Fiji: ApoB analysis aortic root cross sections
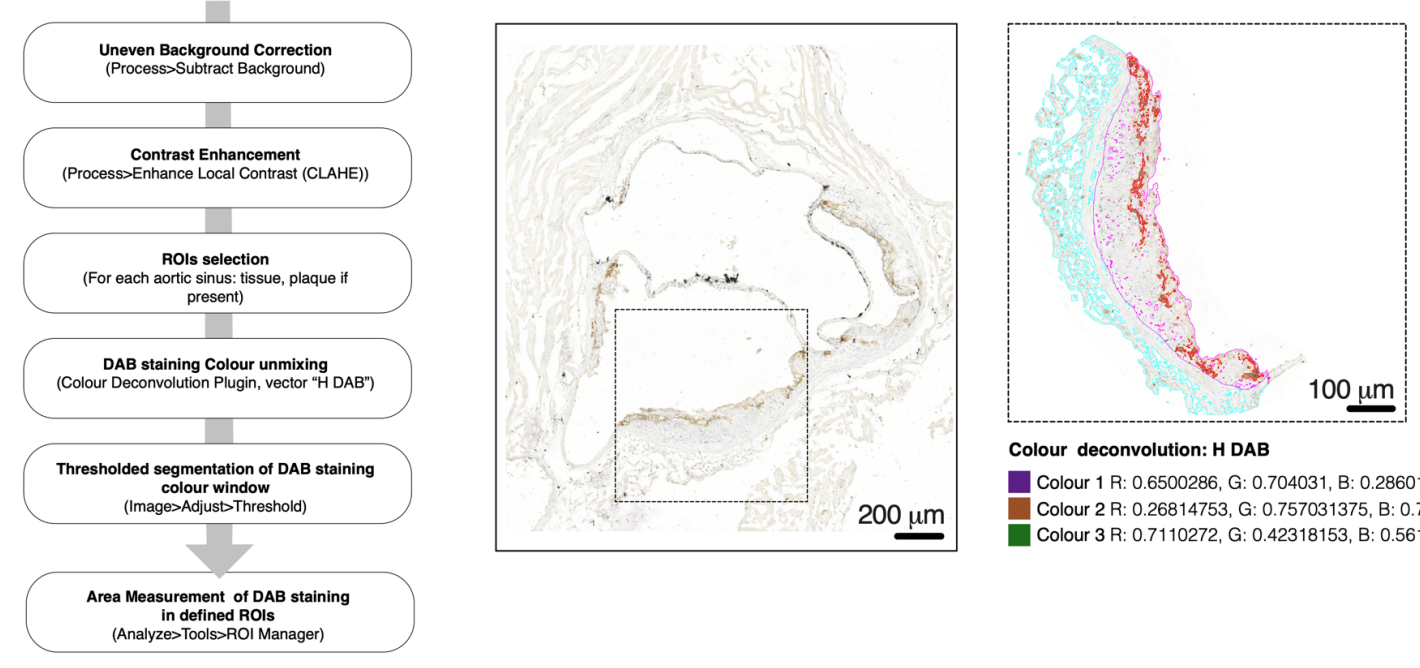

Colour deconvolution: $\mathrm{H}$ DAB

Colour 1 R: 0.6500286, G: 0.704031, B: 0.2860126 Colour 2 R: 0.26814753, G: 0.757031375, B: 0.77642715

Colour 3 R: 0.7110272, G: 0.42318153, B: 0.5615672

Figure 8. ApoB staining analysis in sections of ascending aorta and aortic root. A, Workflow used in Fiji for DAB quantification in ApoB-stained ascending aorta sections. A representative image of a section from a pre-atherosclerotic ascending aorta obtained during the analysis workflow is shown in the overview and insets at higher magnification. Tissue ROI and detected DAB is lined by magenta and red, respectively. Colour Deconvolution "H DAB" shows the color vectors used for color deconvolution. Scale bars, $100 \mu \mathrm{m}$ in overview and $50 \mu \mathrm{m}$ in insets. B, Workflow used for aortic root sections. Representative images obtained during analysis workflow: an overview of an atherosclerotic aortic root section with a marked region in the left coronary sinus, and a higher magnification of the marked region showing the tissue ROI (cyan), plaque ROI (magenta), and detected DAB (red). Scale bars, $200 \mu \mathrm{m}$ in overview and $100 \mu \mathrm{m}$ in insets. 


\subsubsection{Quantification of amount and localization of labeled LDL in en face aortic scans}

For measurements of the total quantity of Atto680-labeled human LDLs in aortic arches, images from the LiCor Odyssey Infrared Imaging System were converted to 8 bit and inverted. The Atto680-LDL positive areas were quantified using pixel threshold segmentation within a user defined ROI (including aortic branches and aortic arch until the first intercostal artery) (Figure 9).

Workflow Fiji: LDL entry and retention analysis en face scans
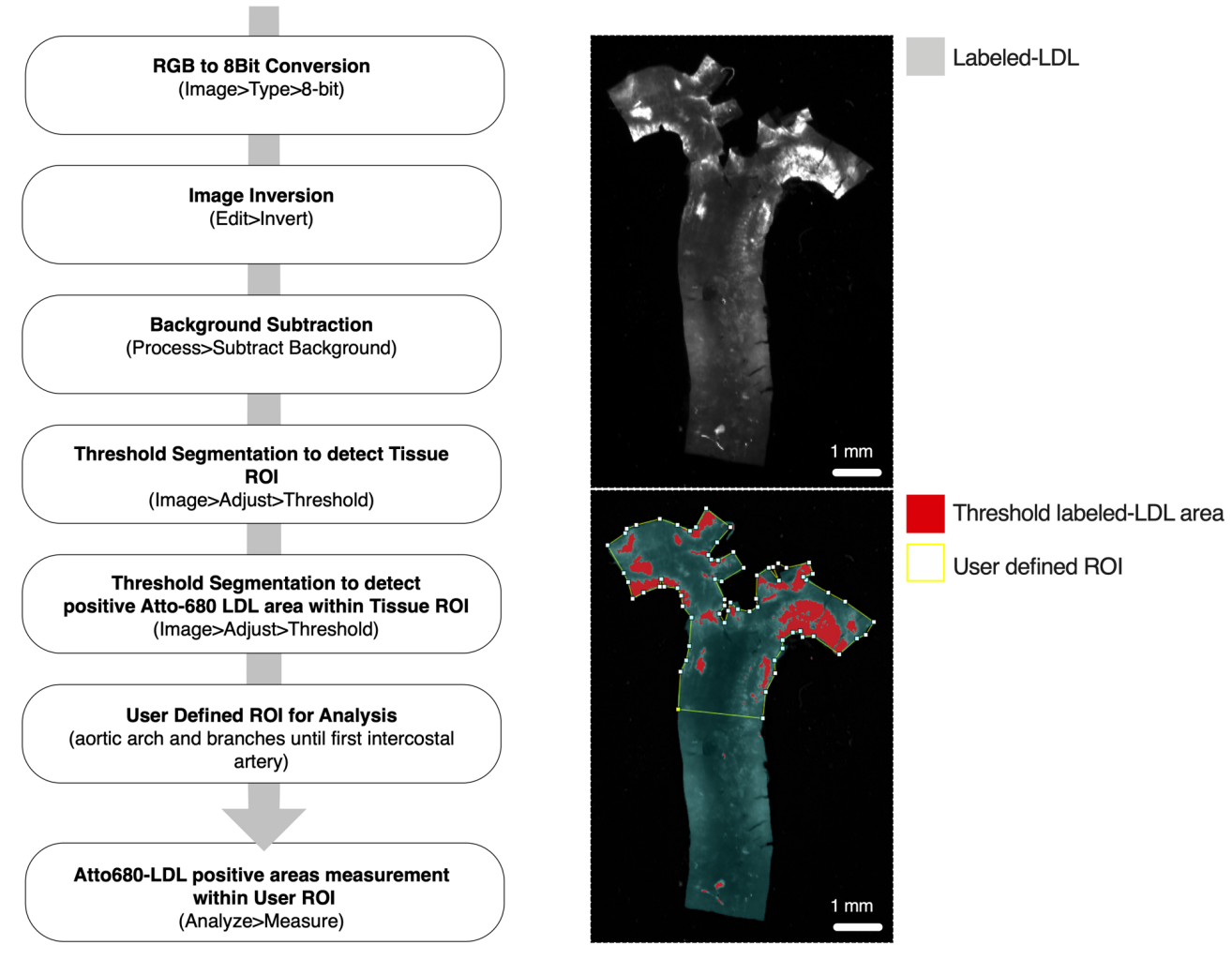

Figure 9. Analysis of Atto680-LDL in en face thoracic aorta scans. Workflow used in Fiji for measuring the area with Atto680-LDL. Representative images obtained during the analysis workflow. The top image shows the inverted image with the gray Atto680 signal. The bottom image shows the pixel threshold segmentation selection of the Atto680-LDL positive area (red) and the user-defined ROI (yellow perimeter). Scale bars, $1 \mathrm{~mm}$.

En face images were further analyzed to map potential differences in the localization of Atto680-LDL between conditions. To this end, an intensity profile was measured along a transverse line (width 3 pixels $=54.54 \mu \mathrm{m}$ ) running from the inner to the outer curvature and placed proximal to the brachiocephalic branch point. The 
transverse line was divided into three zones: central inner (CZ, 0-500 $\mu \mathrm{m})$, border (BZ 500-1000 $\mu \mathrm{m}$ ), and outer ( $\mathrm{OZ}>1000 \mu \mathrm{m}$ ) zone. A broader range was used for defining the border zone than for whole-mount confocal (see below) to account for variability in the placement of the longitudinal cut in the central inner zone used to open the aortic arch for en face scanning. The analysis was always performed on the dorsal part (shown to the left in en face images) of the split ascending aortic arch.

\subsubsection{Quantification of amount and localization of labeled LDL and macrophages in whole-mount confocal images of the ascending aorta}

The analysis of whole-mount confocal microscopy images was focused only on the inner curvature region of the ascending aortic arch. A transverse thick line (width 200 pixels $=83 \mu \mathrm{m}$, length $2000 \mu \mathrm{m}$ ) was placed across the inner curvature region and was further divided into three zones: dorsal border zone (BZ1, -1000 - -650 $\mu \mathrm{m})$, central inner zone $(\mathrm{CZ},-650-+650 \mu \mathrm{m})$, and ventral border zone (BZ2 +650 - +1000 $\mu \mathrm{m})$, with the coordinates referring to the midline of the inner curvature. Negative values correspond to the dorsal side of the ascending aortic arch and positive values to the ventral side.

For intensity profile analysis, the signal in z sections was summed using the sum slices z-projection method on images after applying background correction, and then an intensity profile of Atto647 or Atto565 signal was plotted along the thick line area.

For the co-localization analysis of areas with high LDL entry and areas with high LDL retention, and of high-content macrophage and high LDL retention areas, individual images with LDL entry/retention and Mac-2 stainings were subjected to sum slices z-projection. Because the LDL and Mac-2 signals were captured in separated sessions, the sum projected images were rotated using the BigWarp plugin which allows the matching of tissue shapes using different landmarks as reference, thereby allowing the alignment of separated images of the same specimen. ${ }^{123}$ After the alignment, a straightened new multichannel image containing LDL (entry and retention) and Mac-2 signals was created and used for further analysis. Labeled LDL (Atto647 or Atto565) and Mac-2 (Alexa488) channels were background corrected and their brightness enhanced before pixel thresholding segmentation. Binary mask images were created for both LDL entry (Atto647 or Atto565) and retention channels 
(Atto647 or Atto565), and their individual and co-localization areas were measured. To analyze macrophage accumulation in high LDL retention areas, the area occupied by LDL retention signal was computed for Mac-2 high-content and Mac-2 low-content selections (Figure 10).

Workflow Fiji: co-localization analysis of LDL entry, high-content macrophage and LDL retention areas

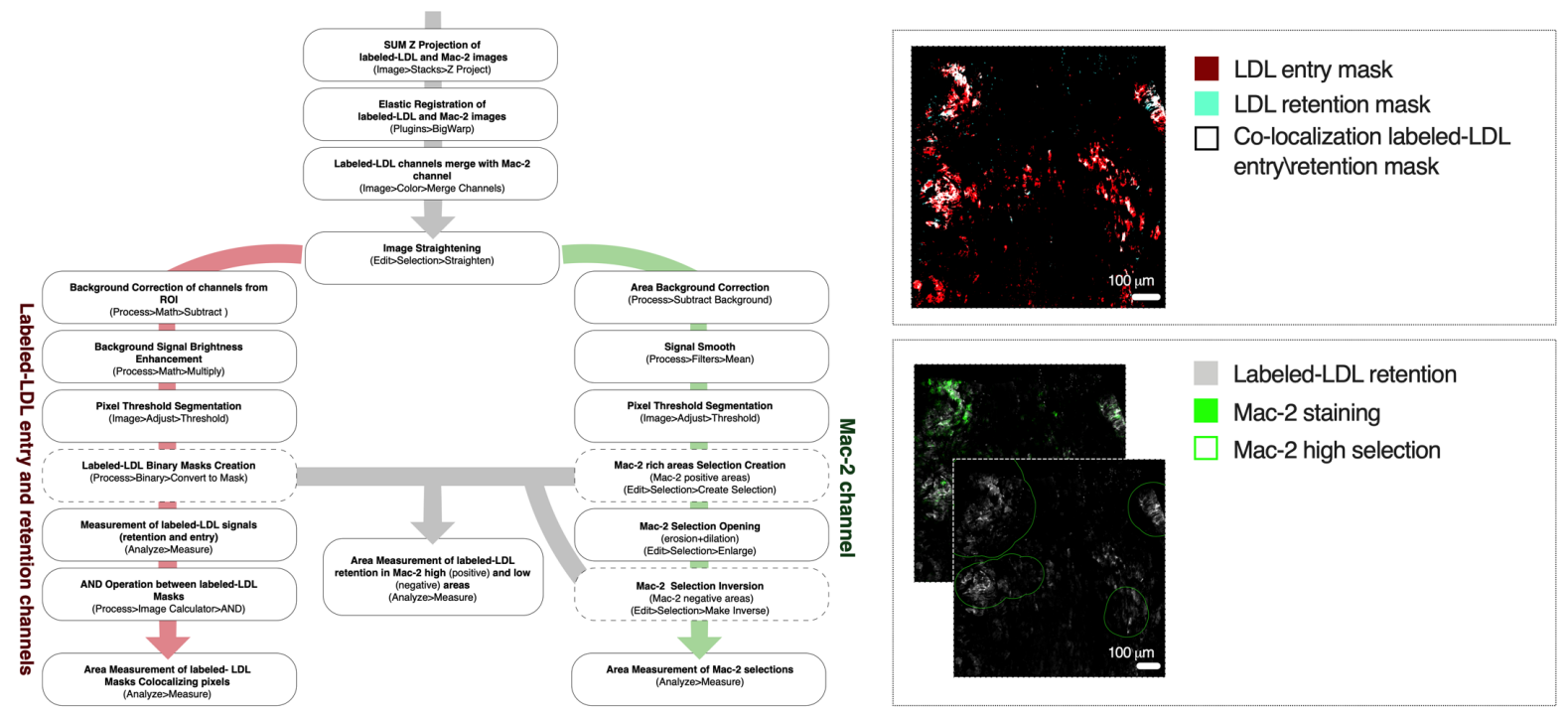

Figure 10. Co-localization analysis of LDL entry/retention areas and LDL retention in high and low content macrophage areas. Left panel, workflow used in Fiji for LDL entry/retention co-localization area and LDL retention in Mac-2 high and low areas measurement. Right panel, representative images obtained during analysis workflow: top, whole-mount image of part of intact inner curvature region of the aortic arch showing LDL entry (red), retention (cyan), and co-localization (white) masks used for quantification. Bottom, whole-mount image of part of intact inner curvature region of the aortic arch showing LDL retention (gray), Mac-2 staining (green), and corresponding Mac-2 high selection (green line) used for quantification. Scale bars, $100 \mu \mathrm{m}$.

\subsubsection{Quantification of LDL retention and macrophage content in sections from ascending atherosclerotic aorta}

To measure the content of retained labeled LDL (Atto647 signal) and of Mac2stained macrophages (Alexa Fluor 568 signal) in serial sections of atherosclerotic ascending aorta, signals in the confocal $\mathrm{z}$ slices were summed and ROls corresponding to tissue and plaque were defined, and their areas quantified. Elastin autofluorescence signal was detected and subtracted from the Atto647 channel, and areas with high levels of Atto647-LDL signal and Alexa Fluor 568-macrophage signal 
were outlined and measured through pixel thresholding segmentation in the plaque ROIs (Figure 11).

Workflow Fiji: labeled-LDL retention and macrophage content analysis in atherosclerotic aortas
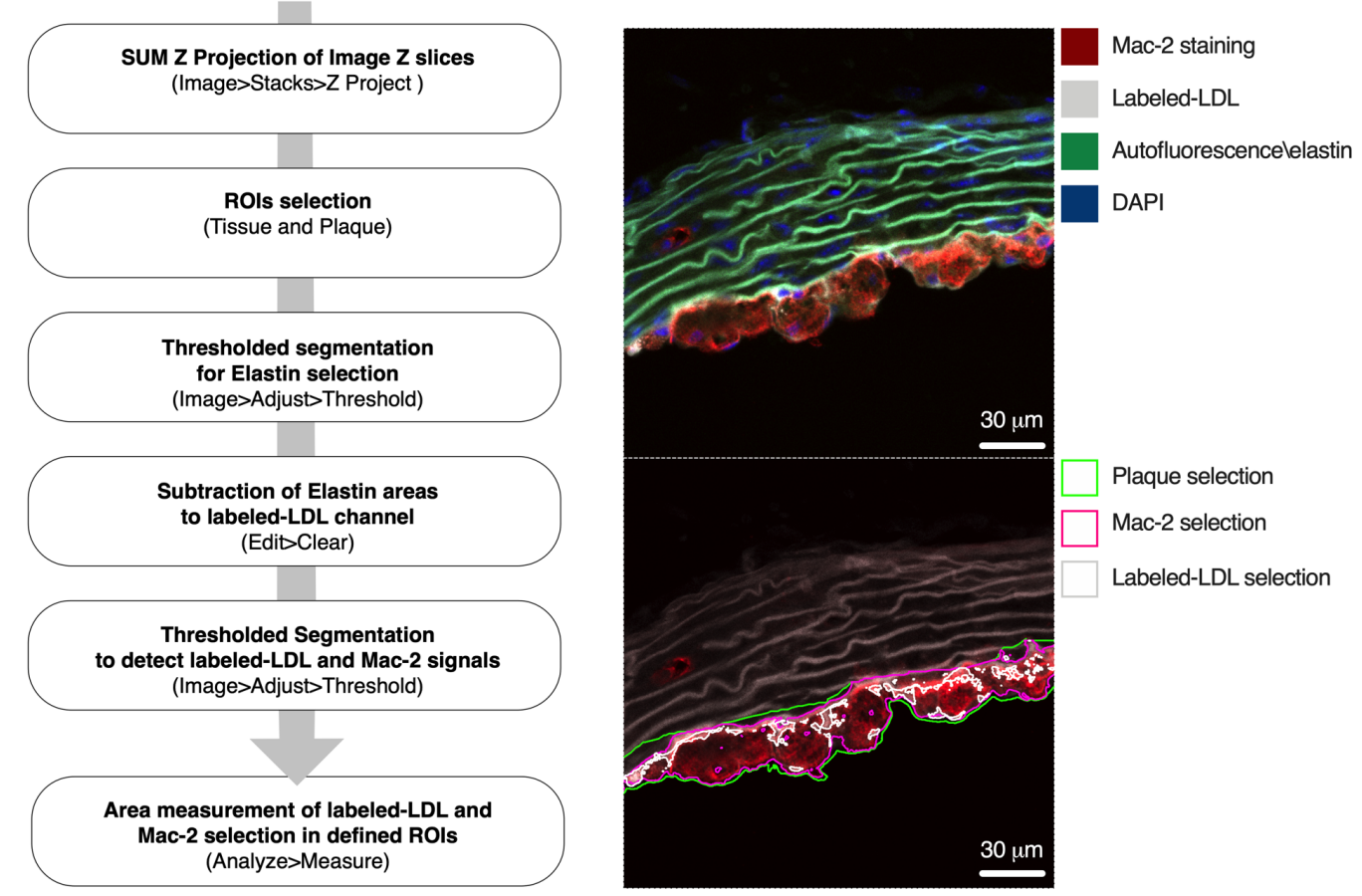

Figure 11. LDL retention and macrophage content analysis in atherosclerotic aortas. Workflow used in Fiji for measurement of areas with LDL retention and macrophages in atherosclerotic ascending aortas. Representative images obtained during analysis workflow are shown. The top image shows an aortic cross section with LDL retention (gray) and Mac-2 staining (red). The bottom image shows the user-defined plaque ROI (light green line), and the detected macrophage (pink line) and labeled-LDL (light gray line) ROIs after pixel segmentation thresholding. Scale bars, $30 \mu \mathrm{m}$.

\subsection{Arterial gene expression profiling by RNA sequencing}

Total RNA was isolated from the atherosclerosis-prone inner curvature region of pre-atherosclerotic and normal aortic arches using the RNeasy Micro Kit (QIAGEN). Tissue disruption and homogenization was performed with TissueRuptor and RNA was treated with DNase I according to the manufacturer's instructions. In each group, RNA from $n=9$ mice was pooled in 3 RNA pools of $n=3$ mice each. RNA quality and quantity were evaluated through Agilent Bioanalyzer 2100 using the RNA 6000 Nano Chip (Agilent Technologies). The Next-Generation Sequencing (NGS) (RNAseq) 
service was performed by the Genomic Unit of CNIC. Briefly, barcoded RNA-seq libraries were prepared using the NEBNext Ultra II Directional RNA Library preparation kit (New England Biolabs) according to manufacturer's instructions. The size of the libraries was checked using the Agilent 2100 Bioanalyzer and the concentration was determined using the Qubit $\AA$ fluorometer (Life Technologies). Libraries were then sequenced on a HiSeq2500 (Illumina) to generate 60 bases single reads. RNA-seq data analysis was performed by the Bioinformatics Unit of CNIC. Briefly, sequencing reads were processed with a pipeline that used FastQC to evaluate their quality and Cutadapt to trim sequencing reads, eliminating Illumina adaptor remains, and to discard reads that were shorter than 30 bp. ${ }^{124,125}$ Resulting reads were mapped against mouse transcriptome GRCm38.91, and gene expression levels were estimated with RSEM. ${ }^{126}$ Around $80 \%$ of the reads participated in at least one reported alignment. Expected expression count matrices were then processed with an analysis pipeline that used Bioconductor package limma for normalization (using TMM method) and differential expression testing. ${ }^{127}$ Changes in gene expression were considered significant if associated with Benjamini and Hochberg adjusted p-value < 0.05. 117 genes were detected as differentially expressed in the performed contrast. An Ingenuity Pathway Analysis (IPA) (QIAGEN Inc., https://www.qiagenbioinformatics.com/products/ingenuity-pathway-analysis) was performed to interpret the differentially expressed data and identify the most significant biological processes, canonical pathways, upstream transcriptional regulators, and gene networks.

\subsection{Statistics}

Statistical tests were performed in Prism 8 (GraphPad Software). Two-sample comparisons were analyzed by the unpaired Student's t-test for normally distributed data and by the Mann-Whitney test for non-normally distributed data. Three or moresamples comparisons were performed with the ANOVA test for normally distributed data and by the Kruskal-Wallis test for non-normally distributed data. Post-tests were used to interrogate differences between groups as noted in the figure legends. Plasma cholesterol was quantified as the area under the curve over the course of experiments. All tests were 2-tailed and differences were considered statistically significant at 
$P<0.05$. Bars in scatter dot plots represent mean \pm SD. All images shown are examples of replicates and are representative for the respective groups. 



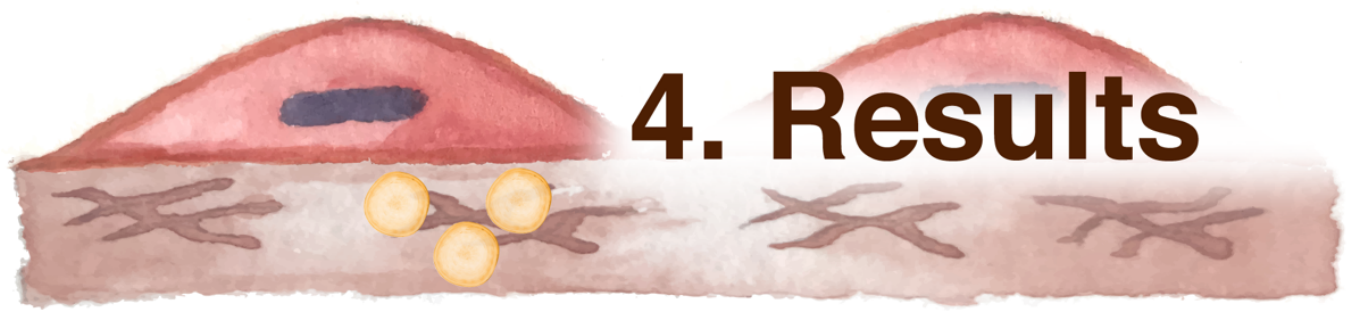




\subsection{LDL accumulation precedes plaque development in mice}

Mouse models with inborn hypercholesterolemia, such as Apoe or Ldlr knockout mice, do not feature a clear starting point for the processes leading to plaque initiation. To study the temporal relationship between hypercholesterolemia onset, intimal LDL accumulation, and atherosclerosis initiation, we subjected 8-week-old wildtype mice to abrupt induction of hypercholesterolemia using virus-mediated transfer of proprotein convertase subtilisin/kexin type 9 (PCSK9) followed by a highcholesterol diet for 2, 4, 6, or 12 weeks ( $n=7$ mice per group) (Figure 12).

A Experimental Design

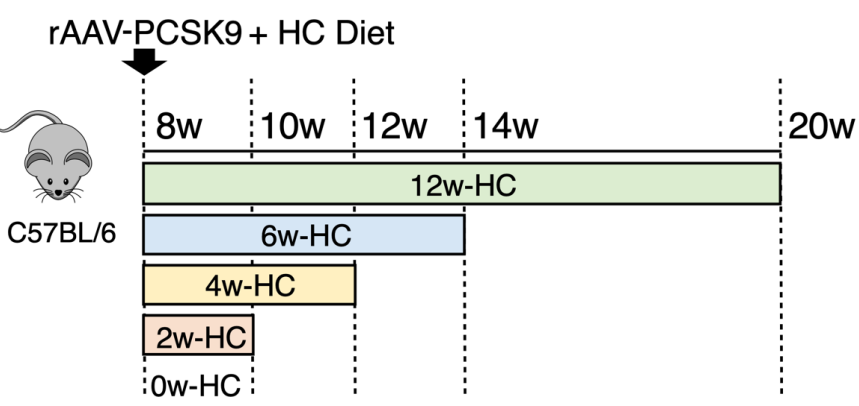

B Plasma LDL Cholesterol

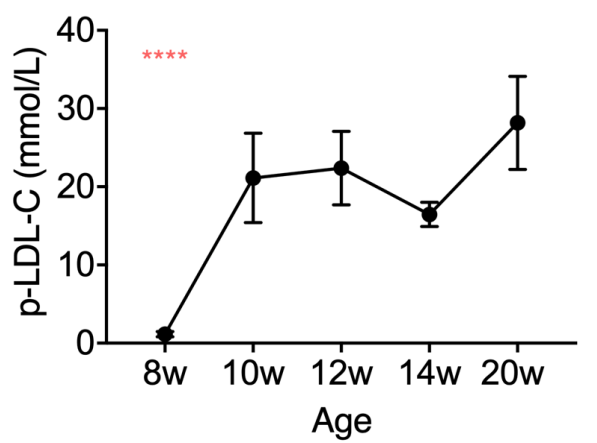

Figure 12. Induction of atherosclerosis through PCSK9 overexpression. A, Experimental design. Groups of 8-week-old mice were injected with rAAV-PCSK9 and fed a high cholesterol $(\mathrm{HC})$ diet for 0 $(n=7), 2(n=7), 4(n=7), 6(n=7)$, or 12 weeks $(n=7)$. B, Plasma LDL cholesterol concentration $(\mathrm{mmol} / \mathrm{L})$ at $8,10,12,14$, and 20 weeks of age. ${ }^{* * *} \mathrm{P}<0.0001$ (Kruskal-Wallis test).

LDL accumulation was quantified in serial cross sections from the distal ascending aorta and the aortic root by staining for Apolipoprotein B (ApoB) (Figure 13). The atherosclerosis-prone ascending aorta already showed significant ApoB accumulation across the inner part of the aortic arch after 2 weeks. This preceded the appearance of the first foam cells which were seen at 4 weeks in some mice, leading to a non-significant increase in measured intima area. Over the following weeks, the initially scattered foam cells progressed to lesions that eventually covered the entire inner aortic arch region. Concurrently, increasing ApoB staining was localized in developing plaques. The outer arch portion did not accumulate LDL or develop lesions over the time period studied. 
A

A 2 re $(O C) \quad 2 w$

$4 w$
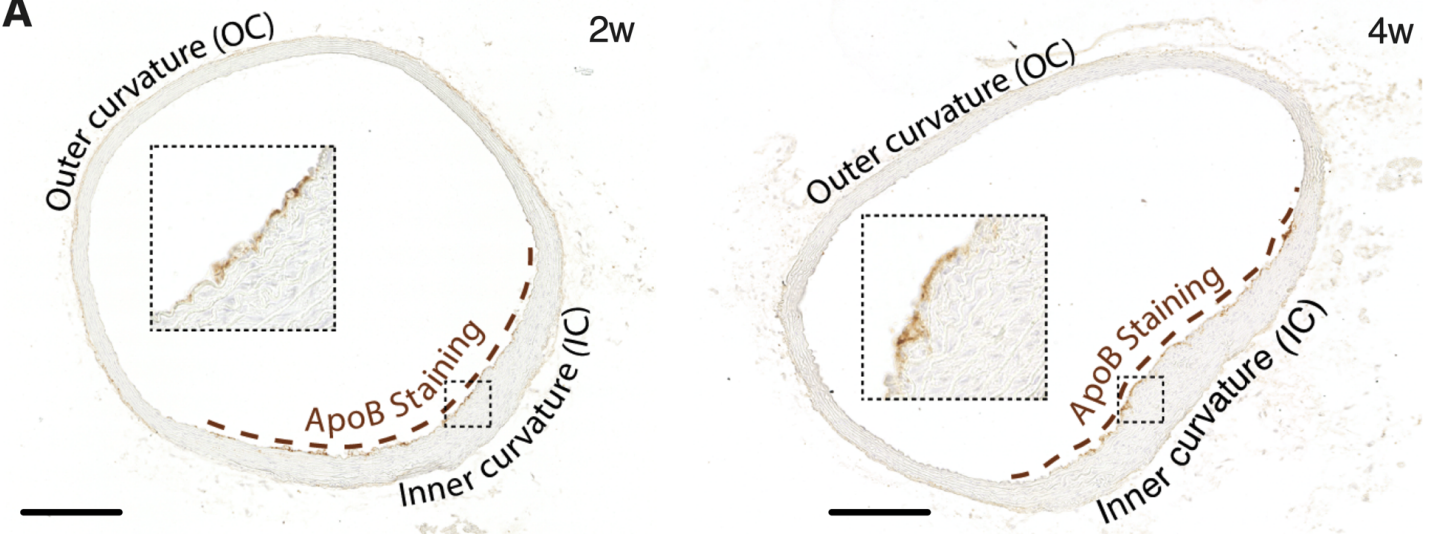

B

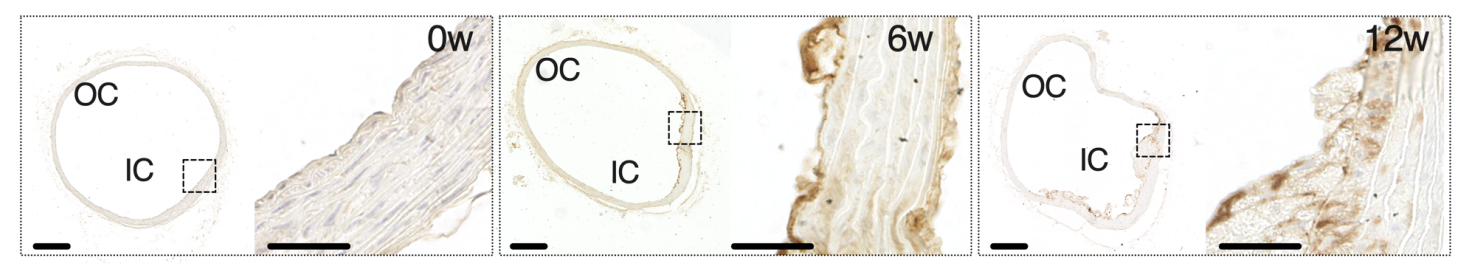

C

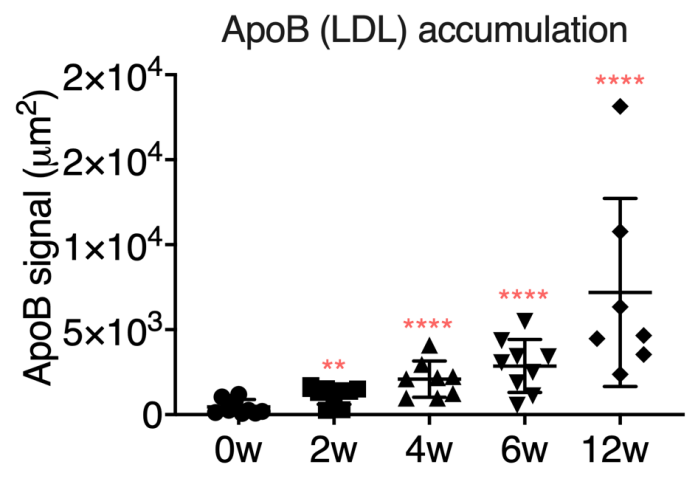

D

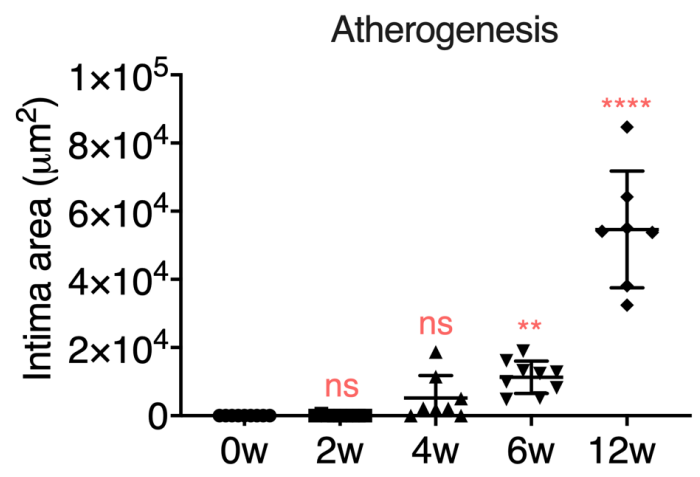

Figure 13. LDL accumulation and atherogenesis in mouse ascending aorta. A-B, LDL accumulation detected by staining for its protein moiety $A p o B$ (brown) precedes plaque formation in the inner arch region of ascending aorta sections from mice subjected to 0,2, 4, 6 and 12 weeks of hypercholesterolemia. Insets show selected areas at higher magnification. Scale bars, $200 \mu \mathrm{m}$ in overview and $50 \mu \mathrm{m}$ in insets. C-D, Quantification of ApoB accumulation and intima (plaque) area. ANOVA of log-transformed data (C) and Kruskal-Wallis test (D) followed by post-test comparison to normal aorta at $0 \mathrm{w}$. ns, non-significant, ${ }^{* *} \mathrm{P}<0.01,{ }^{* * *} \mathrm{P}<0.0001$.

Similar results were obtained in the aortic root. Each of the three sinuses showed significant $A p o B$ accumulation at 2 weeks, and the level of accumulation at this stage correlated with the rate of subsequent lesion development, being highest in the most atherosclerosis-prone left coronary (LC) sinus and lowest in the less atherosclerosis-prone right coronary (RC) sinus (Figure 14). 


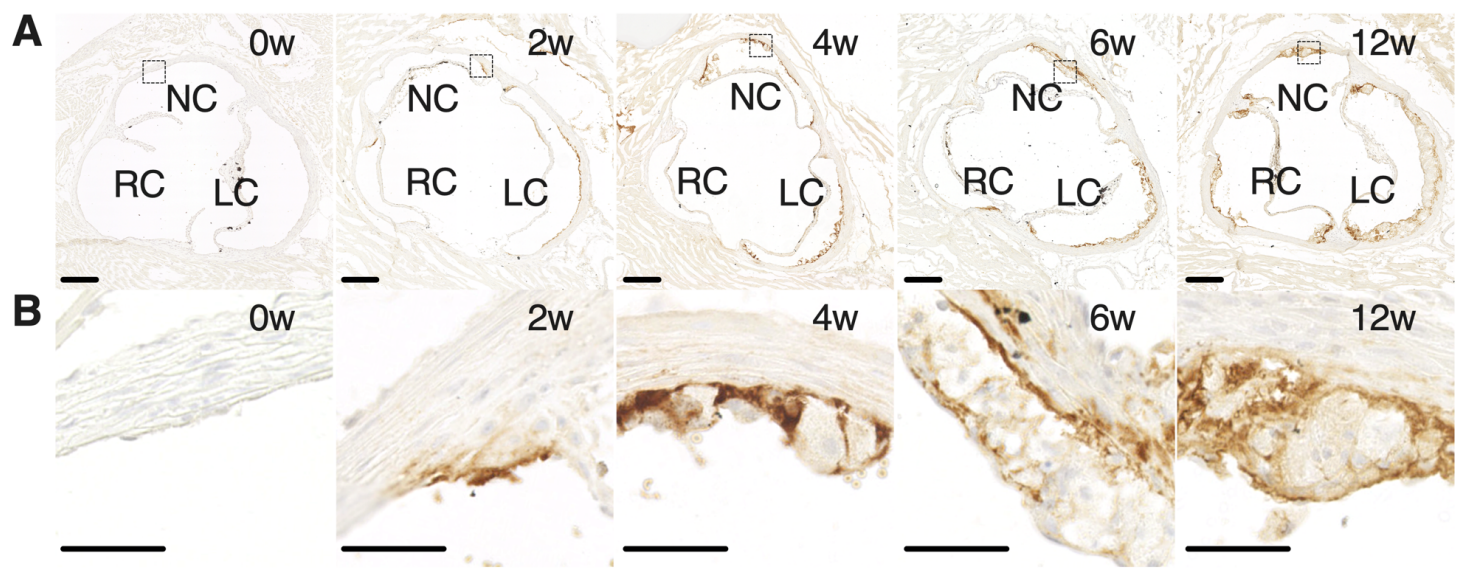

C

D

\section{E}

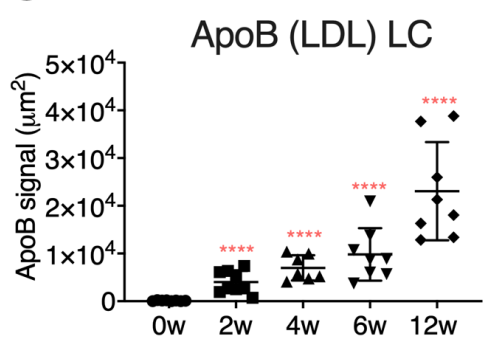

$\mathbf{F}$

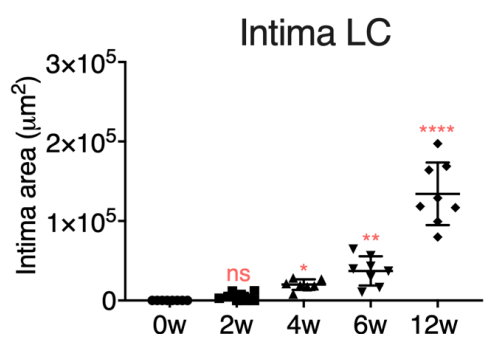

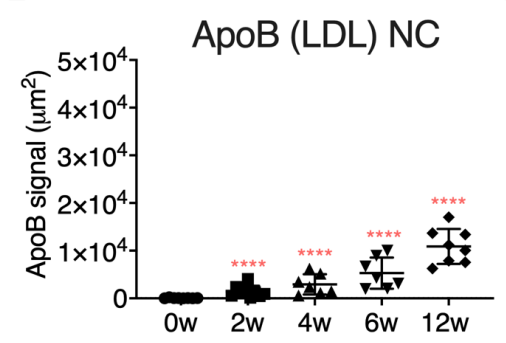

G

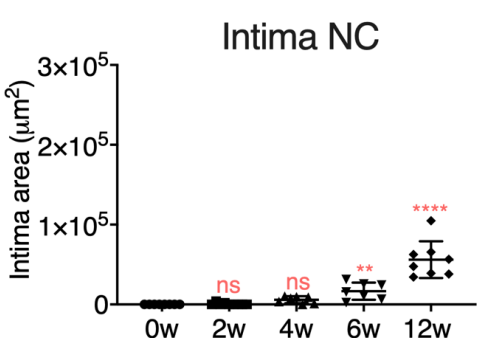

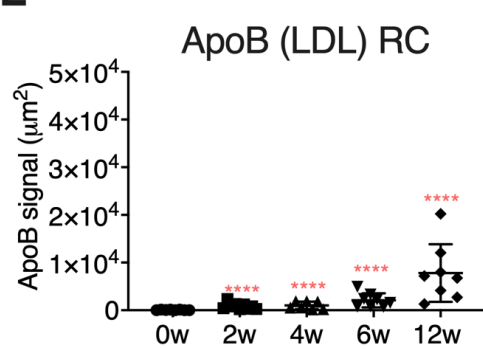

$\mathbf{H}$

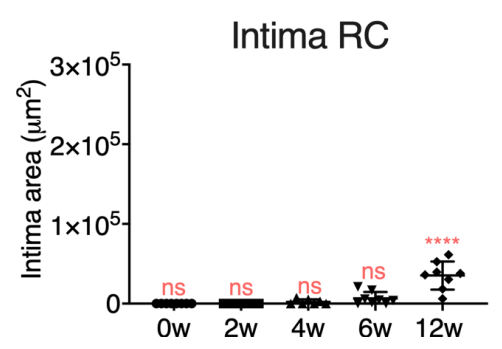

Figure 14. LDL accumulation and plaque development in mouse aortic root. A, Immunostaining for mouse ApoB (brown) in aortic sinus sections from mice injected with rAAV-PCSK9 and fed HC diet for $0 w, 2 w, 4 w, 6 w$, or $12 w$. B, Higher magnification views of the area marked a box in A. C-E, Quantification of $A p o B$ (LDL) accumulation in the left (LC), non- (NC), and right coronary (RC) aortic root sinus over time. F-H, Quantification of intima (plaque) area in each sinus over time. Scale bars, $200 \mu \mathrm{m}(\mathrm{A})$ and $50 \mu \mathrm{m}(\mathrm{B})$. ANOVA of log-transformed data (C, D, E) and Kruskal-Wallis test ( $F, G, H)$ followed by post-test comparison to normal aortic sinus at $0 \mathrm{w}$. ns, non-significant, ${ }^{*} \mathrm{P}<0.05,{ }^{* *} \mathrm{P}<0.01$, ${ }^{* * * *} \mathrm{P}<0.0001$.

Thus, as expected, LDL accumulation in PCSK9-induced mice precedes the initiation of lesions at atherosclerosis-prone sites in a pre-atherosclerotic phase. To understand the processes that regulate LDL accumulation, we then focused on the ascending aortic arch after 3.5 weeks of hypercholesterolemia. This time point is at the end of the pre-atherosclerotic phase at this site; close to the onset of atherosclerosis, but still with very few foam cells present. 


\subsection{Development and validation of high-resolution mapping methods for LDL entry and retention}

Buildup of LDL in the arterial wall is determined by the plasma LDL concentration and the capacity and regulation of LDL entry and retention mechanisms in the arterial wall. To study how LDL retention and entry mechanisms might control LDL accumulation in pre-atherosclerotic arteries, we set up several, including new, techniques to measure these processes using fluorescently labeled LDL following a previously published principle. ${ }^{128}$ Fluorescently-labeled human LDL particles were infused (tail vein injection) into mice followed by arterial flushing and perfusion-fixation after 1 hour or 18 hours. After 1 hour, when high concentrations of labeled LDL remain in the circulation, the amount of labeled LDL in arteries is a measure of the rate of LDL entry. ${ }^{128}$ After 18 hours, labeled LDL is mostly cleared from the plasma, and any LDL detected in arteries is defined as being retained (Figure 15). ${ }^{128}$
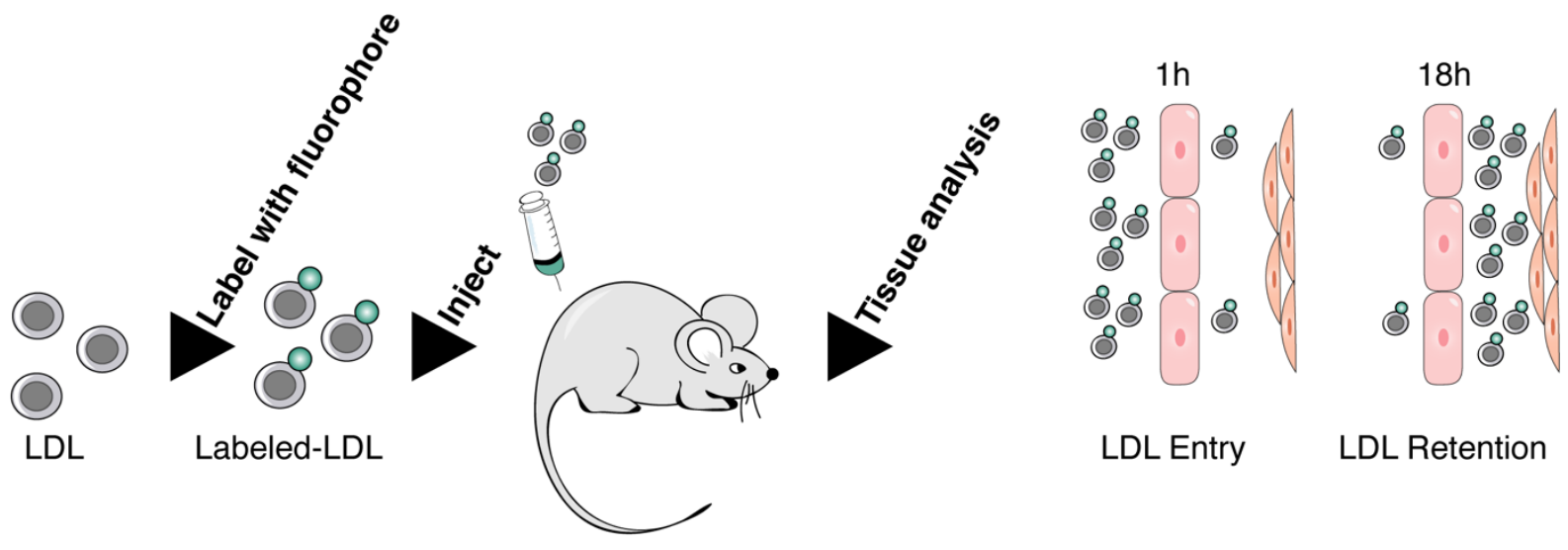

Figure 15. Method used to measure LDL entry and retention in arteries. Mice were injected with labeled LDL and the amount of labeled LDL in the arterial wall was analyzed after 1 hour or 18 hours to measure the rate of LDL entry and LDL retention, respectively.

Figure 16 shows examples of the localization of injected Atto647-labeled LDL in aortic arch cross-sections from mice after 3.5 weeks hypercholesterolemia. After 1 hour, labeled LDL localized around and inside arterial endothelial cells as expected for LDL entering the artery wall, while 18 hours after infusion, labeled LDL localized mainly in the sub-endothelial space as expected for LDL being retained within the artery wall. 

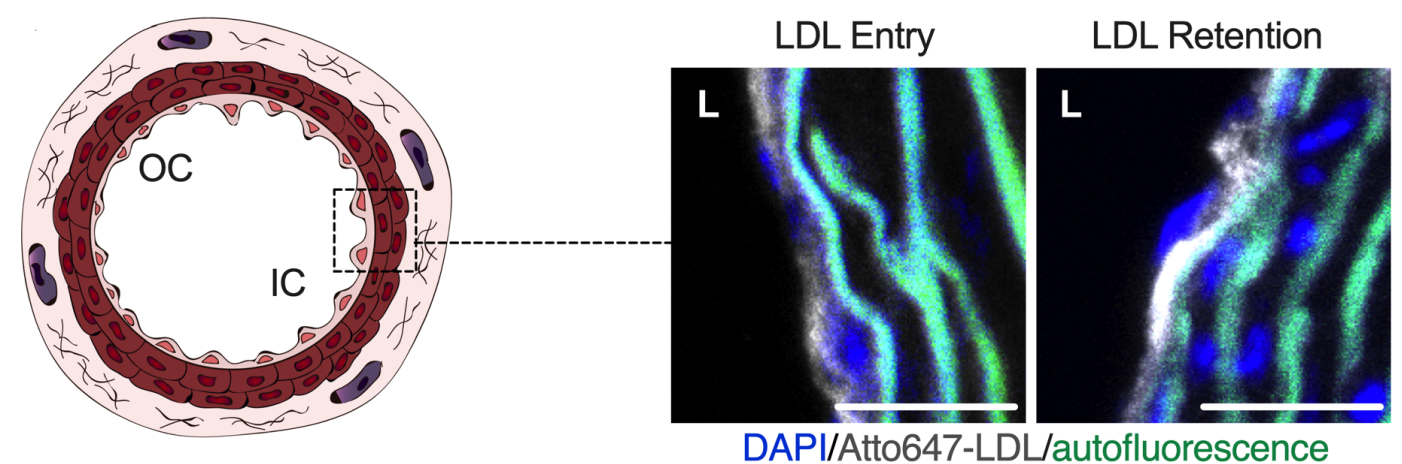

DAPI/Atto647-LDL/autofluorescence

Figure 16. Localization of labeled LDL entered and retained in the artery wall. Aortic arch cross sections showing localization of Atto-647 LDL (grey) 1h (LDL entry) and 18h (LDL retention) after infusion in the artery wall of inner curvature region. DAPI (blue), autofluorescence (green). Similar images were obtained from $n=4$ mice/group. $L=$ lumen, $O C=$ Outer curvature, IC= Inner curvature. Scale bars, $20 \mu \mathrm{m}$.

To produce high-resolution maps of the rates of fluorescent-LDL entry across the aortic arch, we labeled LDL with the near-infrared fluorophore Atto680 and imaged the quantity and location of labeled LDL after 1 and 18 hours by en face infrared (IR) scanning of the opened aorta. Representative pilot scans are shown of the distribution of LDL entry and retention rates across the pre-atherosclerotic surface after 3.5 weeks of PCSK9-induced hypercholesterolemia (Figure 17A). LDL entry and retention were mainly seen at branch points and in the inner curvature region of the aortic arch, but there was reproducible sparing of both entry and retention in the most central zone of the inner arch. The en face scanning technique was previously developed by our research group, ${ }^{72}$ but its merits as a quantitative measure of labeled LDL had not yet been validated. To confirm that scan intensity was proportional to the amount of labeled LDL in the artery, $3.5 \mathrm{w}$ hypercholesterolemic mice were injected with a mix of unlabeled/labeled LDL with increasing percentages of Atto680-labeled LDL (from 0\% to $100 \%$ ). After 18 hours, we measured the amount of retained LDL in the artery and observed a linear relationship between the Atto680 signal and the amount of injected labeled LDL (Figure 18).

As an alternative technique that allows mapping of LDL entry and retention in the same specimen, we developed a whole-mount confocal protocol for imaging aortic arches from mice injected with Atto568-LDL and/or Atto647-LDL at the different time points (Figure 17B). For confocal scans, we found that an alternative approach to opening the aortic arch was preferable to preserve the atherosclerosis-prone inner 
curvature region. The distribution of labeled LDL was consistent with the en face scanning results; high entry and retention were detected at the brachiocephalic branch point (not shown in figure) and in a border zone separating the inner curvature from the rest of the artery, whereas less signal was detected in the central zone of the inner aortic arch.

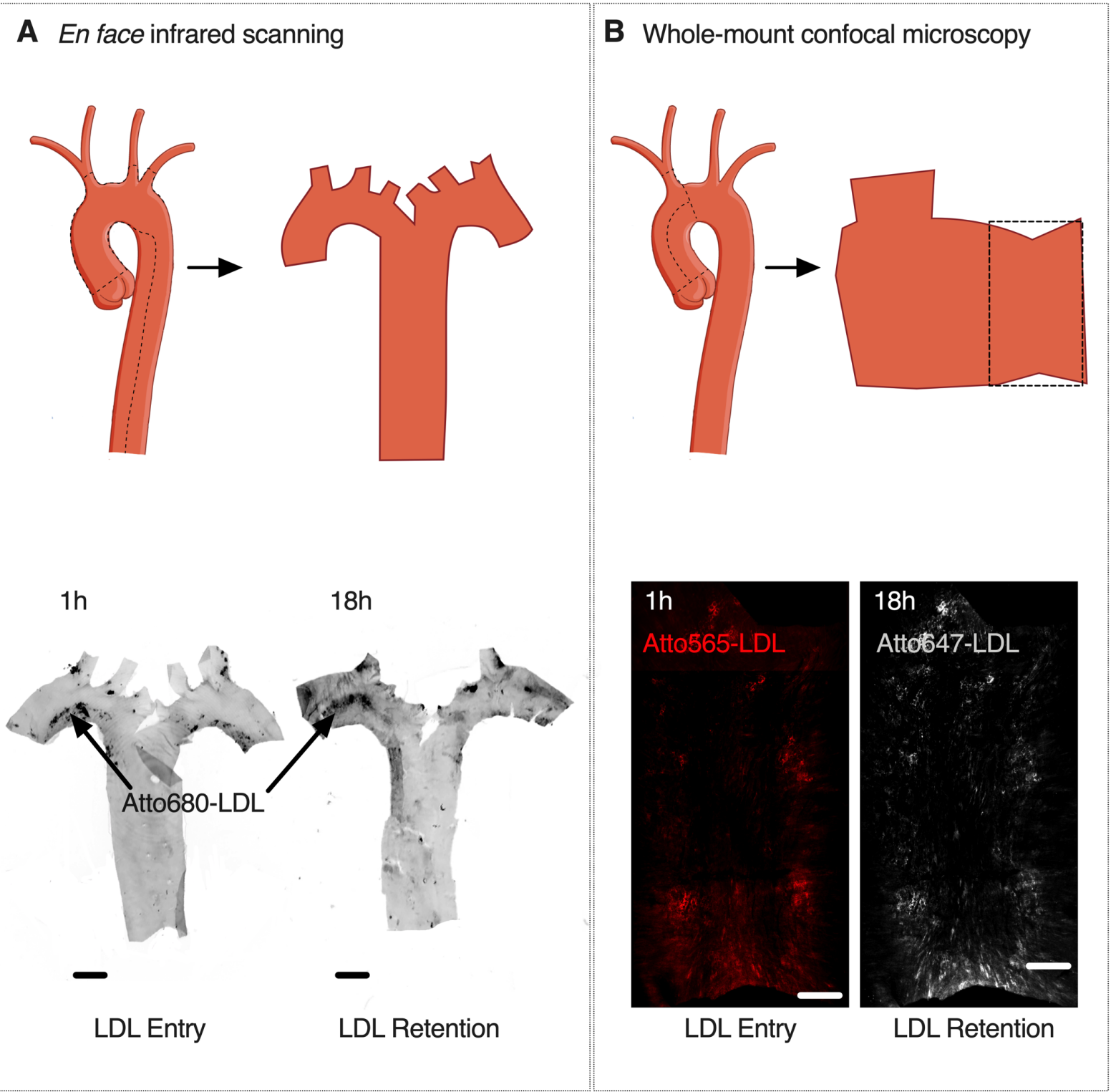

Figure 17. LDL entry and retention measurement techniques. A, En face scanning of arterial Atto680-LDL content. Aortas were opened as indicated and scanned with an infrared scanner. Representative images show the rates of Atto680-LDL entry and retention (black dots) across the aortic arch in 3.5 weeks hypercholesterolemic mice. Scale bars, $1 \mathrm{~mm}$. B, Whole-mount confocal microscopy of Atto565/647-LDL content. Aortas were opened as indicated. Representative images of the aortic inner curvature region show the distribution of Atto565-LDL entry (red) and Atto647-LDL retention (gray) across the aortic arch in 3.5 weeks hypercholesterolemic mice. Scale bars, $200 \mu \mathrm{m}$. 
A

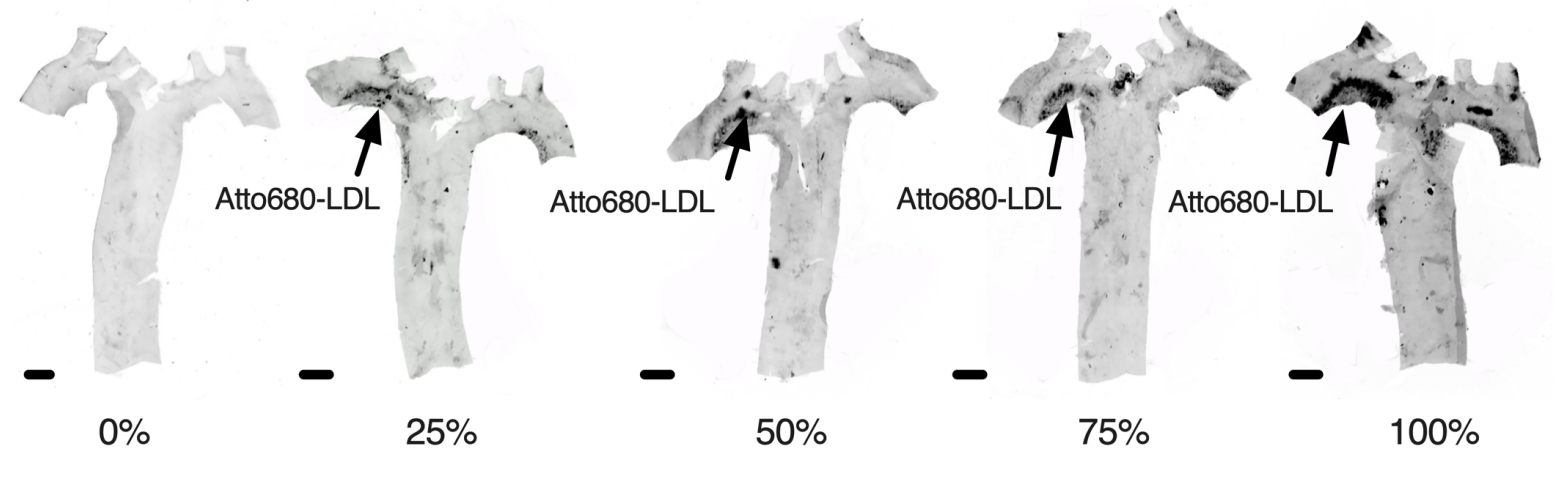

B LDL Retention signal
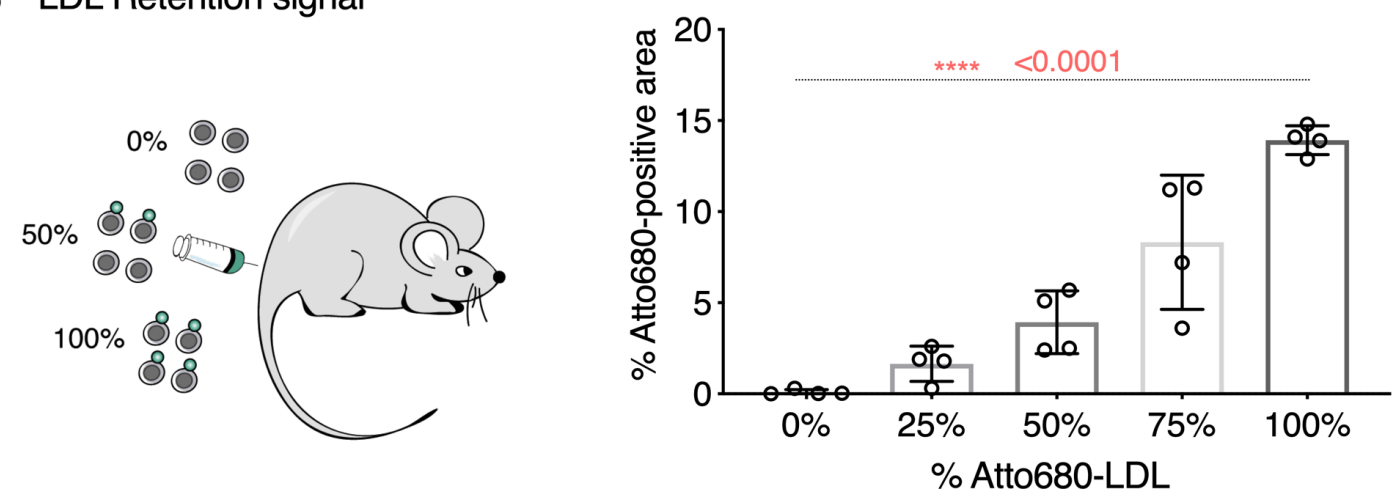

Figure 18. Linear relationship between fluorescent signal and arterial wall content of Atto680LDL. A, Mice with different amounts of retained Atto680-LDL were created by injecting $500 \mathrm{mg}$ of a mix of unlabeled-labeled LDL with increasing percentages of Atto680-LDL ( $n=4$ per group). Scale bars, 1 $\mathrm{mm}$. The images show representative en face scans of aortas of 3.5 weeks hypercholesterolemic mice, harvested at $18 \mathrm{~h}$ after the injection. Scale bars, $1 \mathrm{~mm}$. B, Quantification of images showing a linear relationship between the amount of injected Atto680-LDL and the fluorescence signal. $P^{* * * *}<0.0001$ (Ordinary one-way ANOVA).

\subsection{PCSK9 does not directly affect LDL entry or retention}

PCSK9 has been shown to bind both LDL and proteoglycans, ${ }^{129,130}$ raising the possibility that PCSK9 could act as a bridging molecule in LDL retention. Apart from its clear relevance to understanding PCSK9 function, such an interaction could bias measurements of LDL retention when using PCSK9 as a tool to manipulate plasma LDL levels. To test for this possibility, we injected rAAV-PCSK9 or rAAV-empty control vectors into groups of $L d l r$ knockout $\left(L d l r^{-/-}\right)$mice and compared entry and retention of Atto680-LDL after 3.5 weeks (Figure 19A). 
A Experimental Design
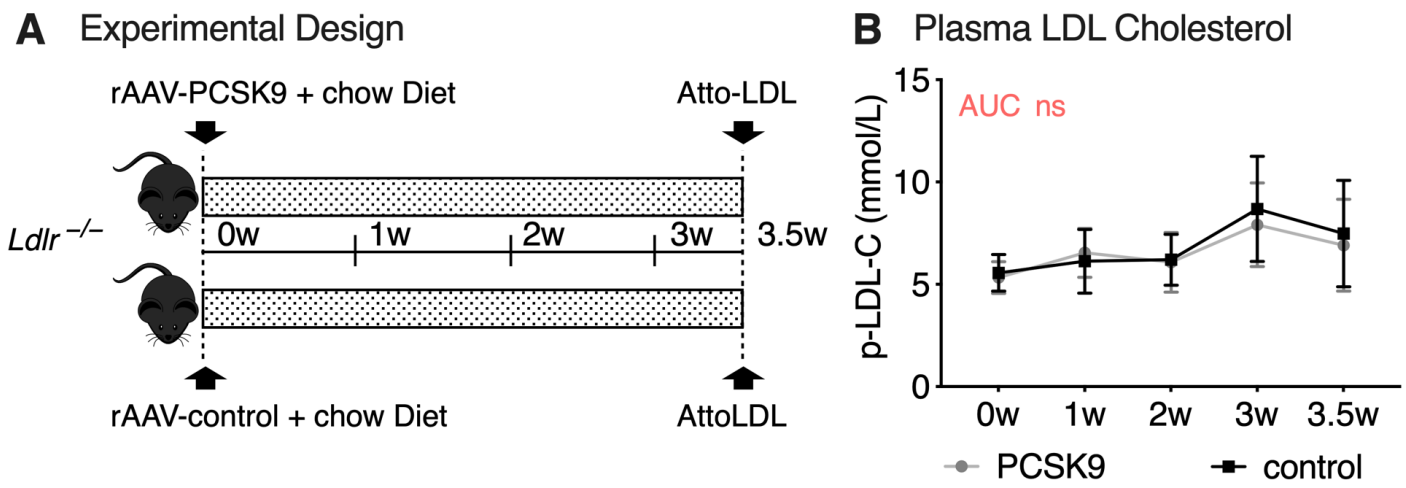

\section{LDL Entry}
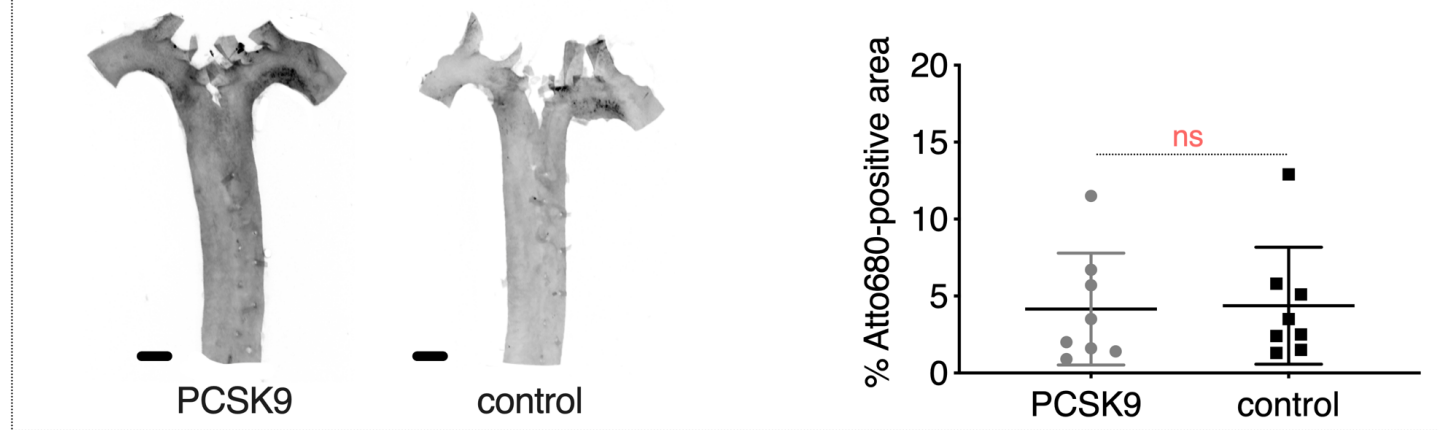

D LDL Retention
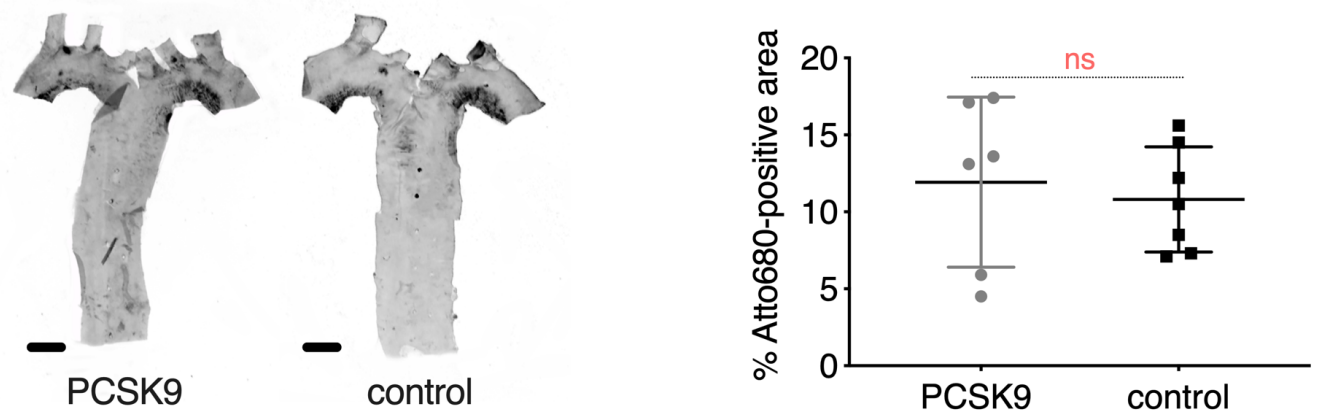

Figure 19. Atto680-LDL entry and retention in LdIr knockout mice injected with rAAV-PCSK9 or rAAV-empty viral vectors. A, Experimental design. $L d l r^{-/-}$mice were injected with rAAV-PCSK9 (PCSK9 group) or a similar rAAV-control without the PCSK9 sequence (control group) and fed standard chow diet for 3.5 weeks. They were then injected with Atto680-LDL at 1 hour ( $n=6$ PCSK9 and $n=7$ control mice) or 18 hours ( $n=8$ PCSK9 and $n=8$ control mice) before termination to measure LDL entry and retention, respectively. B, Plasma LDL cholesterol concentration (mmol/L) in PCSK9 $(n=6)$ and control $(n=7)$ mice. ns, non-significant for comparison of the area-under-the-curve (AUC) (MannWhitney test). C, Atto680-LDL entry at 1 hour post-infusion showing no significant difference between the two groups ( $n=6$ PCSK9 and $n=7$ control mice). ns, non-significant (Mann-Whitney test). D, Atto680LDL retention at 18 hours post-infusion showing no significant difference between the two groups $(n=8$ PCSK9 and $n=8$ control mice). ns, non-significant (Mann-Whitney test). 
Consistent with PCSK9 causing hypercholesterolemia by lowering hepatic LDL receptor content, we found no effect on plasma LDL concentration in mice lacking the LDLR (Figure 19B). Quantification of aortic en face scans of Atto680-LDL also showed no significant effect of PCSK9 on LDL entry or retention (Figure 19C-D). The similar Atto680-LDL entry and retention observed between the two groups confirmed that PCSK9 overexpression can be used to study arterial LDL accumulation during hypercholesterolemia without undue interference.

\subsection{LDL retention is rate limiting for LDL accumulation in pre- atherosclerotic arteries}

After developing tools to map arterial LDL uptake in the aortic arch of mice with PCSK9-induced hypercholesterolemia, we applied them to investigate whether rates of LDL entry or retention are changed in pre-atherosclerotic arteries compared with the normal arterial wall. The accumulation of LDL might itself influence the mechanisms of entry and retention creating positive or negative feedback loops that could shape the course of LDL accumulation and atherosclerosis onset. To address that question, comparison of PCSK9-overexpressing and wild-type mice was not an option, since the vast difference in hepatic LDL clearance would affect the plasma levels of labeled LDL and hence the quantification of LDL retention and entry. Therefore, we treated two groups of mice with rAAV-PCSK9 and the high-cholesterol diet, but in one of the groups we kept plasma LDL at normal levels by treating with antisense oligonucleotides targeting Apoliporotein B (ApoB ASO) (Figure 20A-B). The $A p o B$ ASO promotes hepatic $A p o B$ mRNA degradation, leading to a reduction in hepatic very-low-density lipoprotein (VLDL) output and hence lower plasma LDL levels. The other group was treated with a control antisense oligonucleotide (Ctrl ASO). After 3.5 weeks with high or normal plasma LDL, we conducted the LDL retention and entry assays after injection of the same Atto680-LDL dose in both groups. Clearance of the Atto680-LDL between the pre-atherosclerotic and normal mice was confirmed to be comparable at both 18 hours and 1 hour after injection (Figure 20C).

The total rate of LDL entry and retention across the aortic arch was measured by the en face technique. Total entry of Atto680-LDL was significantly higher while 
total retention was significantly lower in mice after 3.5 weeks of hypercholesterolemia (pre-atherosclerotic) compared with normocholesterolemic mice (Figure 21).

A Experimental Design

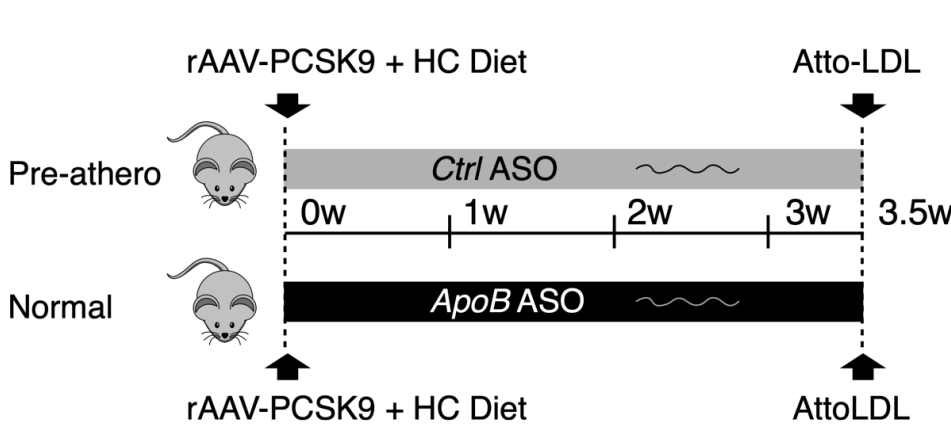

B Plasma LDL Cholesterol

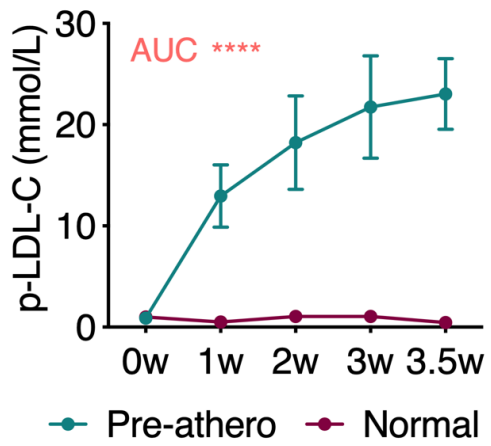

C Labeled-LDL Plasma Clearance

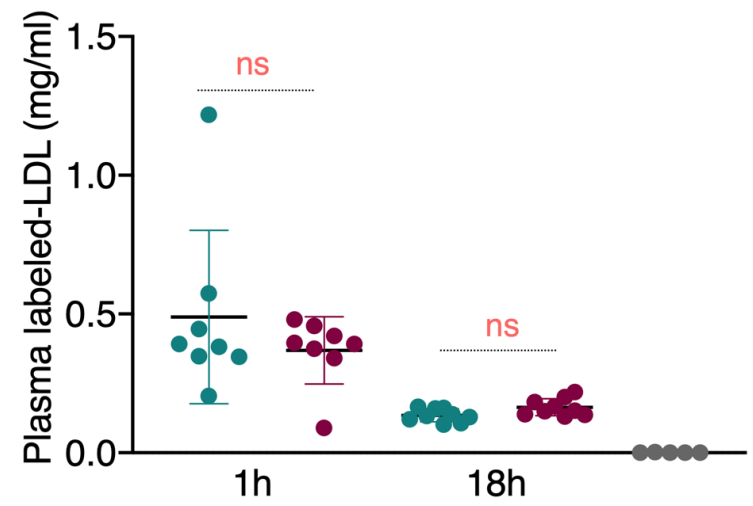

Figure 20. Experimental design to compare LDL entry and retention in pre-atherosclerotic and normal arteries. A, By combining rAAV-PCSK9, high cholesterol $(\mathrm{HC})$ diet and $A p o B$ or Control antisense oligonucleotides (ASOs), mice were assigned to high LDL cholesterol (pre-atherosclerotic arteries/Ctrl ASO) or normal LDL cholesterol (normal arteries/ApoB ASO) groups. Entry and retention of injected fluorescent-LDL were measured after 3.5 weeks. B, Plasma LDL cholesterol concentration (mmol/L) in pre-atherosclerotic $(n=13)$ and normal aortas $(n=15) .{ }^{* * * *} \mathrm{P}<0.0001$ (Mann-Whitney test) comparing area-under-the-curve (AUC). C, Level of labeled-LDL was measured in plasma with a fluorometer and was found to be similar between groups both at $1 \mathrm{~h}$ ( $\mathrm{n}=8$ mice/group) and $18 \mathrm{~h}$ ( $\mathrm{n}=9$ mice/group) after infusion indicating similar $L D L$ clearance rates. Additional mice $(n=5)$ mice were not infused with labeled LDL as a control. ns, non-significant (Mann-Whitney test).

These findings show that the rate of LDL accumulation is not simply determined by plasma LDL levels but involves changes in both entry and retention mechanisms. The total entry of LDL in the pre-atherosclerotic phase was even higher than predicted by the increase in plasma LDL levels, whereas the amount of total retained LDL was lower than expected. These two observations combined suggest that the global capacity of the artery wall for LDL retention is saturated in hypercholesterolemic mice 
and that retention is the overall rate-limiting step for LDL accumulation during the preatherosclerotic phase.

\section{A LDL Entry}

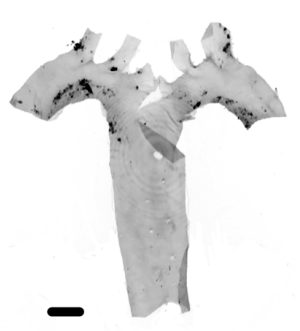

Pre-athero
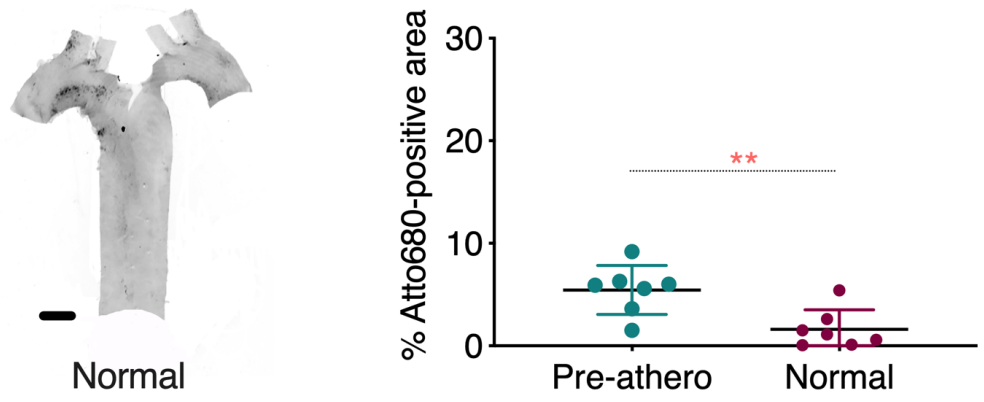

B LDL Retention

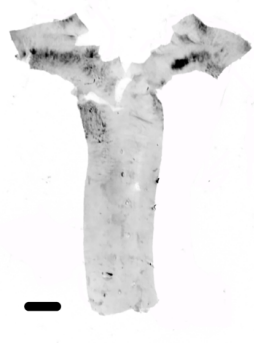

Pre-athero

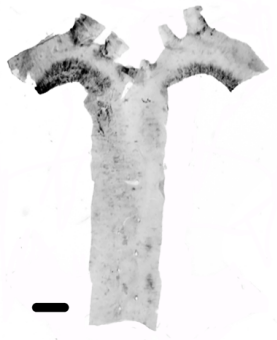

Normal

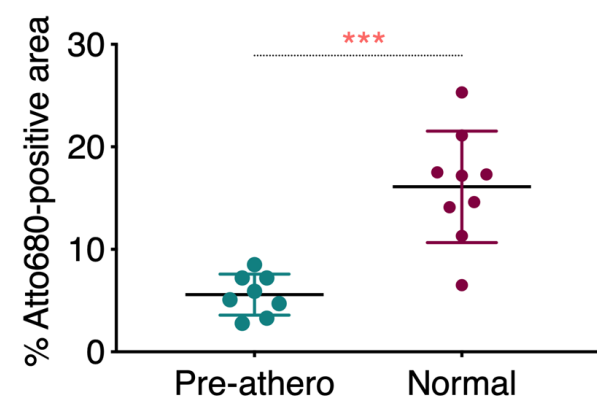

Pre-athero Normal

Figure 21. Global LDL retention is reduced despite increased entry into the pre-atherosclerotic aorta. A, Total Atto680-LDL entry in pre-atherosclerotic $(n=7)$ and normal aortas $(n=7)$ at 1 hour after infusion. ${ }^{* *} \mathrm{P}<0.01$, Mann-Whitney test. $\mathrm{B}$, Total Atto680-LDL retention in pre-atherosclerotic $(\mathrm{n}=8)$ and normal aortas $(n=9)$ at 18 hours after infusion. ${ }^{* * *} P<0.001$ (Mann-Whitney test). Scale bars, $1 \mathrm{~mm}$.

\subsection{Rates of total LDL entry, but not retention, increase during the pre-atherosclerotic phase}

To corroborate these results with an alternative approach, we analyzed how total LDL entry and retention rates change during the first weeks after the onset of hypercholesterolemia. For this, we treated mice with rAAV-PCSK9 injection and initiated the high-cholesterol diet 4 weeks, 2 weeks, or 1 week before measuring Atto680-LDL entry and retention by the en face technique (Figure 22A-B). The 
experimental design was chosen so that assays could be performed with the same batch of Atto680-LDL to allow direct comparison.

A Experimental Design

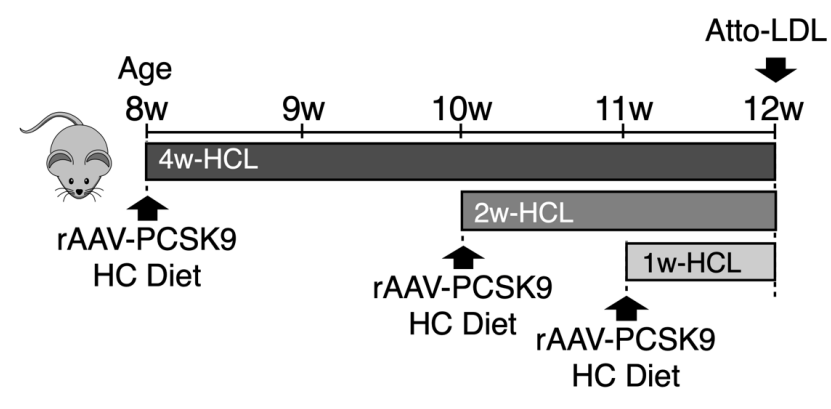

C LDL Entry

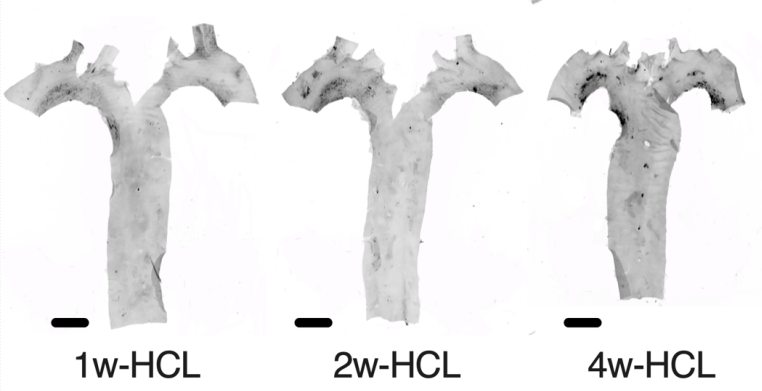

D LDL Retention

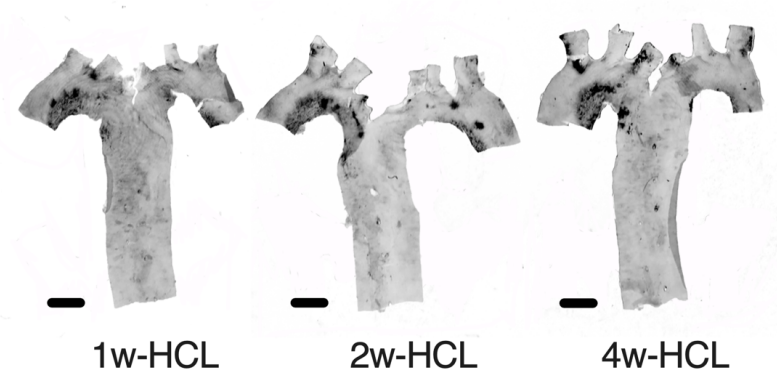

B Plasma LDL Cholesterol

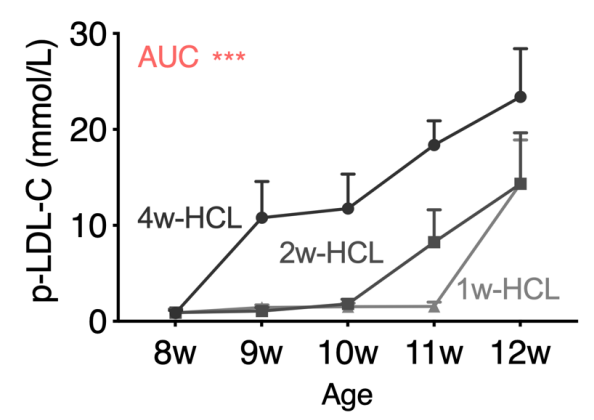

Figure 22. Progression of total LDL entry and retention during the pre-atherosclerotic phase. A, Experimental design. Groups of mice were injected with rAAV-PCSK9 and fed a high cholesterol (HC) diet for 1, 2, or 4 weeks before measurement of LDL entry and retention at 12 weeks of age. B, Plasma LDL cholesterol concentration ( $\mathrm{mmol} / \mathrm{L}$ ) in the three groups at $8,9,10,11$ and 12 weeks of age. ${ }^{* * *} \mathrm{P}<0.0001$ (Kruskal-Wallis test) comparing area-under-the-curve (AUC). C, Atto680-LDL entry after 1 hour in thoracic aortas exposed to hypercholesterolemia $(H C L)$ for $1(n=9), 2(n=9)$, and $4(n=7)$ weeks. Quantification of total signal shows an increasing rate of total LDL entry during the pre-atherosclerotic phase. ${ }^{*} \mathrm{P}<0.01$ (Kruskal-Wallis test). $\mathbf{D}$, Atto680-LDL retention after 18 hours in pre-atherosclerotic thoracic aortas exposed to hypercholesterolemia for $1(n=7), 2(n=7)$, and $4(n=8)$ weeks. Quantification of total signal shows no changes in the rate of total LDL retention during the pre-atherosclerotic phase. ns, non-significant (Kruskal-Wallis test). 
Significant increases in the total rate of Atto680-LDL entry were observed during the pre-atherosclerotic phase (Figure 22C), but this was not paralleled by increases in the total rate of retention (Figure 22D). This result confirms that the rate of LDL retention, but not that of LDL entry, is the limiting factor for total LDL accumulation in the mouse aortic arch during the pre-atherosclerotic phase.

\subsection{Gene expression changes in pre-atherosclerotic arteries}

The differences in LDL entry and retention rates of pre-atherosclerotic compared with normal arteries could potentially be caused by upregulation of genes facilitating endothelial transcytosis and downregulation of LDL retaining matrix molecules, respectively. To probe for such changes, we measured gene expression at the atherosclerosis-prone inner aortic curvature region in the presence of normal and high LDL levels through RNA-seq. We used the same experimental set up shown in Figure 20, but after 3.5 weeks we isolated the inner curvature region of preatherosclerotic and normal arteries for total RNA isolation and high-throughput sequencing (Figure 23).

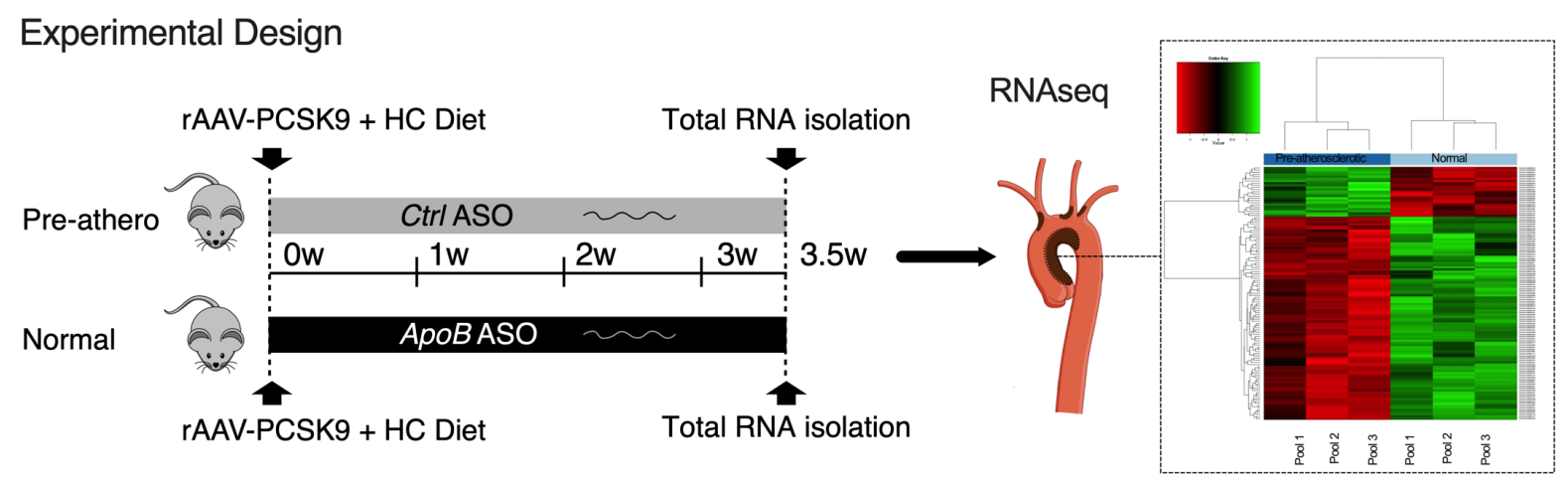

Figure 23. Analysis of gene expression changes in pre-atherosclerotic arteries. Mice were injected with rAAV-PCSK9 and fed high cholesterol $(\mathrm{HC})$ and then treated with either ApoB ASO to reduce plasma LDL to normal levels $(n=9)$ or a Ctrl ASO to maintain hypercholesterolemia $(n=9)$ for 3.5 weeks. RNA was isolated from the inner curvature region of the aortic arch, pooled ( 3 pools of $n=3$ mice/group), and sequenced (RNA-seq).

We identified 117 differentially expressed genes with an adjusted Pvalue of $<0.05$. In the hypercholesterolemic setting, 23 genes were upregulated and 94 downregulated (Figure 24A). 
A Differentially Expressed Genes (Adjusted P-value <0.05)

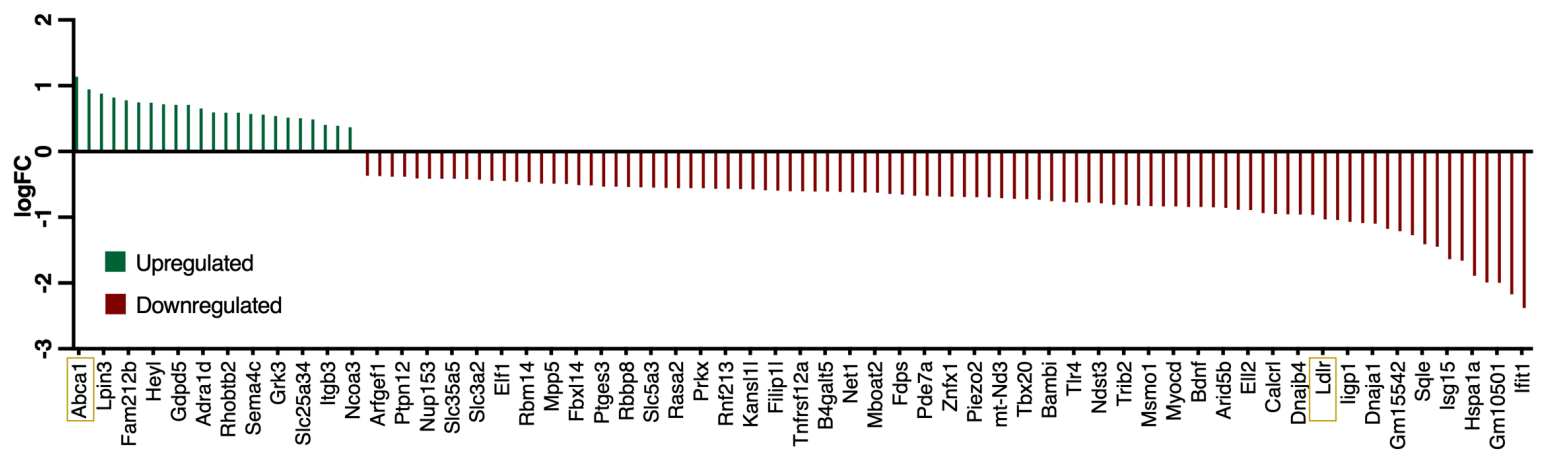

B Ingenuity Pathway Analysis (IPA)

Molecular and Cellular Functions
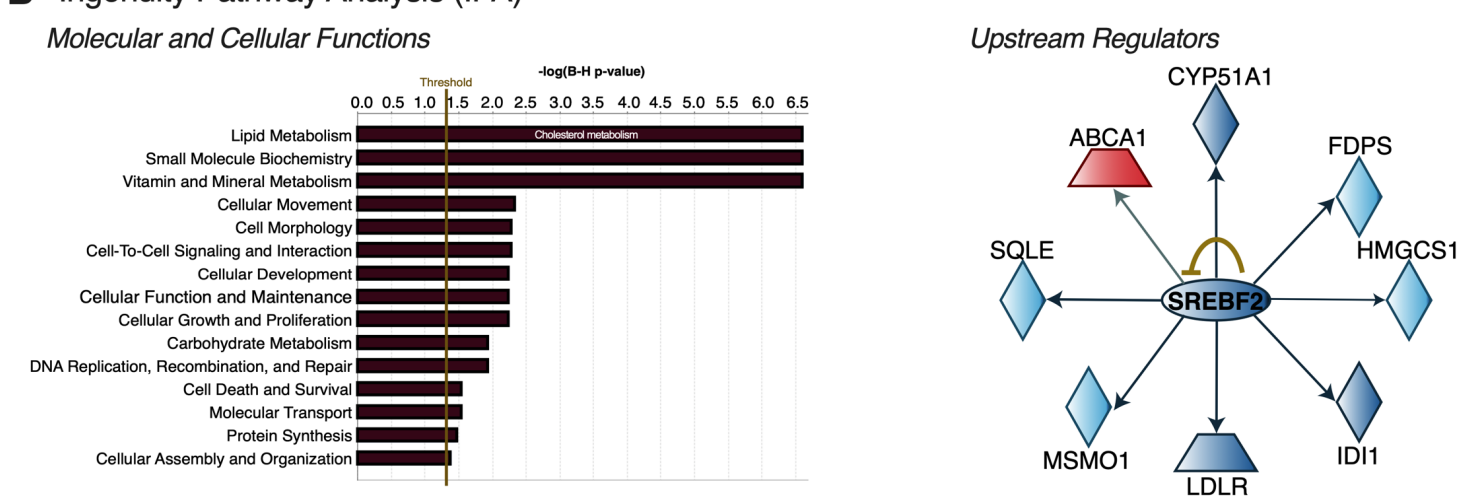

Figure 24. Regulated genes in pre-atherosclerotic arteries. A, RNA-seq data showing the 117 differentially expressed genes (Adjusted P-value $<0.05$ ) in the atherosclerosis-prone inner curvature region after 3.5 weeks of hypercholesterolemia. B, Ingenuity Pathway Analysis (IPA) of the identified differentially expressed genes showing an over-representation of genes involved in lipid metabolism. Srebpf2 was found to be the most significant predicted upstream regulator.

Among the upregulated genes we identified Abca1, a pivotal regulator of cholesterol efflux. Among the downregulated we identified LdIr, known to be downregulated in cells by cholesterol overload. The Ingenuity Pathway Analysis (IPA) of our differentially expressed genes, showed, among the significant affected cellular functions, an over-representation of lipid metabolism with the most significant annotation being Cholesterol metabolism (Figure 24B). Among the most significant predicted upstream regulators that might explain the changes observed in our data, the ingenuity pathway analysis identifies Srebf2, whose inhibition would explain the upregulation of $A b c a 1$ and downregulation of $L d l r .{ }^{131,132}$ These data are consistent with cholesterol overload of arterial cells that may reduce the intracellular capacity to degrade LDL, therefore facilitating the accumulation of LDL in the sub-endothelial space until saturation. However, this suggestion is only speculative and requires further investigation. 
We did not identify differential expression of genes that have been described to be directly involved in LDL retention, such as the LDL-binding proteoglycans versican, perlecan, decorin, biglycan, syndecan-4, and LPL or LDL aggregating enzymes, such as sphingomyelinase or lipoprotein associated phospholipase A2. Neither did we identify changes in genes for caveolae proteins and endothelial receptors involved in arterial LDL transcytosis, such as Caveolin-1 (CAV-1) and Scavenger receptor class B type 1 (SR-B1) and the Activin receptor-like kinase (ALK-1). This could suggest that the increase in total LDL entry and saturable total LDL retention observed at the atherosclerosis-prone inner curvature region of pre-atherosclerotic arteries, may be caused by indirect mechanisms, such as saturation of the capacity of retention or increases in paracellular endothelial transport. However, this experiment has the limitation of analyzing global gene expression changes occurring in all the cells within the artery wall of the inner curvature region, which may prevent from identifying potential differentially expressed genes within the arterial endothelial compartment or inner media and at the exact sites of LDL accumulation. Endothelial RNA-seq or single-cell RNA-seq (scRNA-seq) of the arterial wall may yield further insights on the gene expression changes that characterize the pre-atherosclerotic phase.

\subsection{Changes in LDL entry and retention are heterogeneous across the aortic arch}

Further inspection of the en face scans used to measure LDL entry and retention in pre-atherosclerotic and normal arteries (Figure 21) suggested that the global measurements did not capture the changes occurring in local regions of the aortic arch. As already observed in the pilot scans (Figure 17), pre-atherosclerotic aortic arches did not show homogeneous high levels of LDL entry and retention in the inner curvature region, although this area is generally thought to be uniformly atherosclerosis-prone. The intensity was higher in longitudinal zones on the ventral and dorsal aortic arch wall (from here on border zones) that lie between the central zone of the inner arch (from here on central inner zone) and the outer curvature region (from here on outer zone). The sparing of the central inner zone in hypercholesterolemic mice was unexpected since $A p o B$ immunostaining detected LDL accumulation in the entire inner arch at 2 to 4 weeks of hypercholesterolemia 
(Figure 13A). Moreover, the pattern appeared different from that of normal aortas, in which retention was more uniform across the central part of the inner curvature region. These observations raised the possibility that not only the level but also the topography of retention may change as arteries accumulate LDL.

To quantify this, we divided the dorsal leaf of the opened aortic arch into central inner, border, and outer zone, defined as indicated in Figure 25A. We then analyzed the intensity profiles of the LDL entry and retention signals in these zones in the en face scans of pre-atherosclerotic and normal arteries shown Figure 21.

A Zones ascending aortic arch

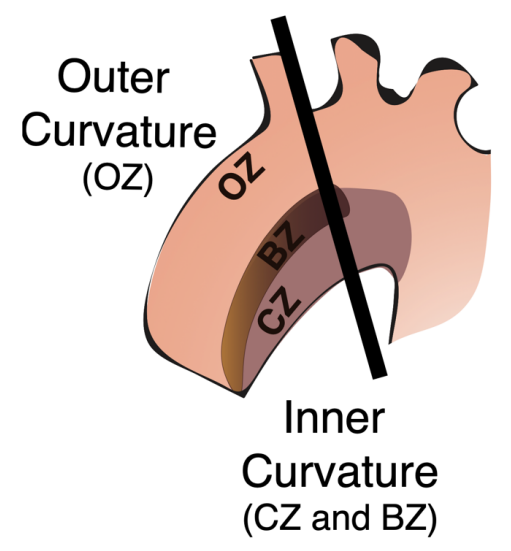

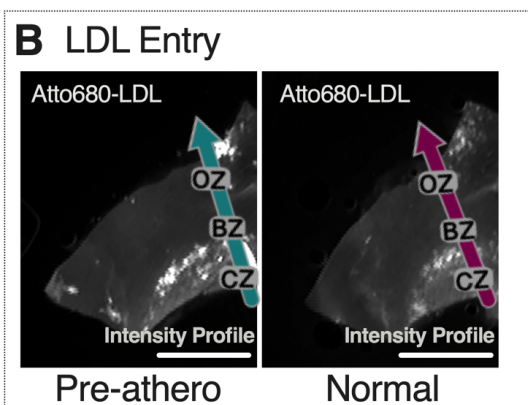
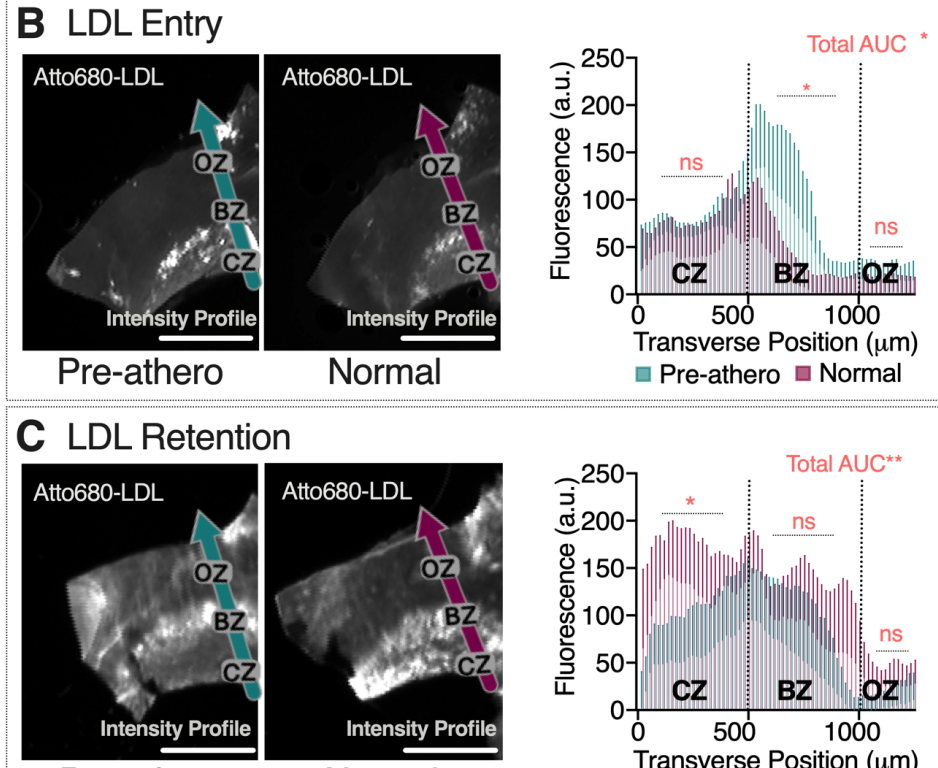

Pre-athero

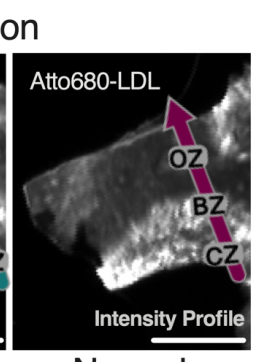

Normal

Figure 25. Topography of LDL entry and retention distribution across the aortic arch in en face

scans. A, Schematic representation of the division of the ascending aortic arch in zones: central inner zone (CZ), border zone (BZ), and outer zone (OZ). B, En face analysis (inverted grey-scale) of Atto680LDL entry in pre-atherosclerotic $(n=8)$ and normal $(n=7)$ arteries, along the zones of the dorsal aortic arch. Intensities were measured along a perpendicular line proximal to the branch point of the brachiocephalic artery. Mean intensity was increased overall (total area-under-the-curve, AUC), and this was explained by increased intensity in the border zone. Graphs show mean $\pm S D$. ns, nonsignificant, ${ }^{*} \mathrm{P}<0.05$ for AUC (Mann-Whitney test). Scale bars, $1 \mathrm{~mm}$. C, En face analysis of Atto680LDL retention, showing overall significantly lower LDL retention in pre-atherosclerotic aortas $(n=6)$ than in normal aortas $(n=9)$, explained by reduced retention in the central inner zone. Graphs show mean \pm SD. ns, non-significant, ${ }^{*} \mathrm{P}<0.05,{ }^{* *} \mathrm{P}<0.01$ for AUC (Mann-Whitney test). Scale bars, $1 \mathrm{~mm}$.

Compared with normal aortas, pre-atherosclerotic aortas showed significantly higher Atto680-LDL entry in the border zone of the inner arch (Figure 25B). Conversely, retention was unchanged in the border zone of pre-atherosclerotic 
compared with normal aortas and was even significantly lower in the central inner zone (Figure 25C). This shows that even though LDL accumulates throughout the inner curvature, the dynamics of LDL entry and retention differ greatly across the aortic arch. In the central inner zone (CZ) of the pre-atherosclerotic aortic arch, there is moderate LDL entry and saturated LDL retention; in contrast, in the border zone (BZ), LDL entry is high and retention mechanisms are not saturated.

To corroborate these observations, we analyzed the topographical pattern of entry and retention by whole-mount confocal microscopy in new groups of mice after 3.5 weeks of hyper- or normocholesterolemia (following again the experimental design shown in Figure 20). In this case, the analysis focused only on the border and central zones of inner curvature region (Figure 26A).

A Zones ascending aortic arch

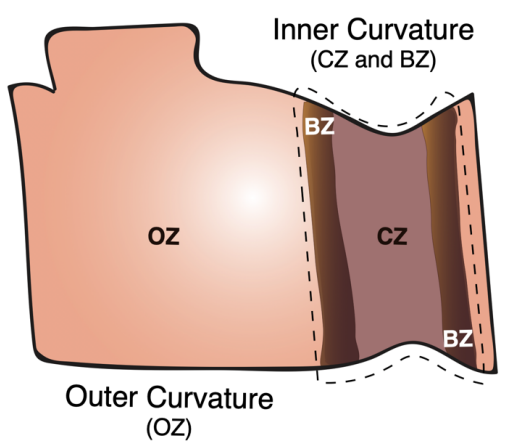

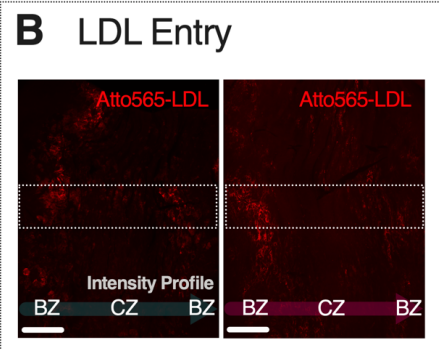
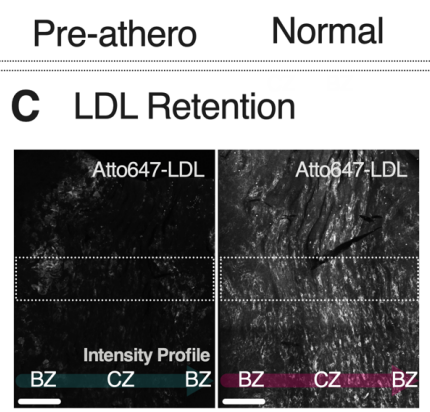

Pre-athero
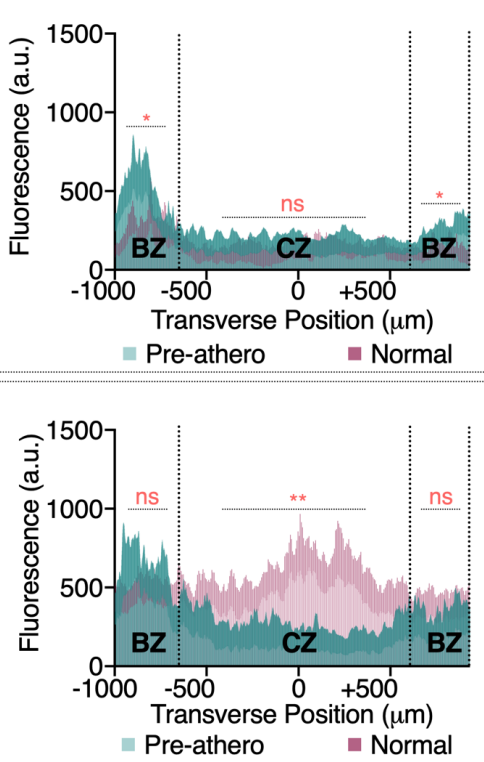

Figure 26. Topography of LDL entry and retention distribution across the aortic arch in wholemount images. A, Schematic representation of the inner part of the aortic arch divided into zones: dorsal border zone (BZ), central inner zone (CZ) and ventral border zone (BZ). B, Whole-mount confocal microscopy distribution of labeled LDL (red) in the inner aortic arch region of preatherosclerotic (left) and normal mice (right). Intensity profiles across the thick line indicate higher entry in the border zones of pre-atherosclerotic aortas $(n=9)$ than in the equivalent regions of normal aortas $(n=6)$. Graphs show mean \pm SD. ns, non-significant, ${ }^{*} P<0.05$ for AUC (Mann-Whitney test). Scale bars, $500 \mu \mathrm{m}$. C, Whole-mount confocal microscopy analysis of retained labeled LDL (gray) showing lower retention in the central inner zone of pre-atherosclerotic aorta $(n=9)$ than in the equivalent region of normal aortas $(n=6)$, where the retention is uniform across the central inner and border zones. Graphs show mean $\pm S D$. ns, non-significant, ${ }^{* *} P<0.01$ for AUC (Mann-Whitney test). Scale bars, $500 \mu \mathrm{m}$. 
To enable analysis of entry and retention in the same aortas, mice were injected with two types of labeled LDL: half the mice received Atto647-LDL at 18 hours before and Atto565-LDL at 1 hour before sacrifice and the rest vice versa. This design was chosen to control for potential differences in fluorescence intensity in the colocalization analysis. Visual inspection and measured intensity profiles confirmed that entry and retention rates were higher in the border zones than in the central inner zone of pre-atherosclerotic aortas, whereas in normal aortas, retention was more uniform across the inner curvature region (central and border zones) (Figure 26B-C).

Consequently, pre-atherosclerotic aortas showed significantly higher colocalization between LDL entry and retention than normal aortas (Figure 27A). To explore what characterizes these sites of high entry and retention in preatherosclerotic aortas, we post-stained aortas for macrophages. Macrophages were predominantly found in the border zones of the inner curvature region, and areas with macrophages had significantly increased levels of LDL retention (Figure 27B).

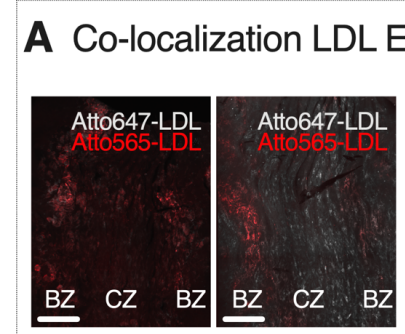

Pre-athero
Normal

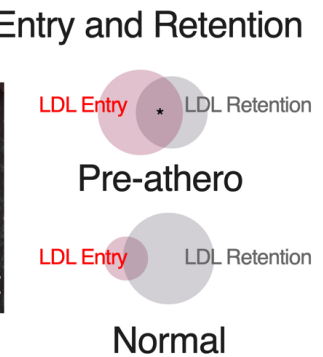

Normal

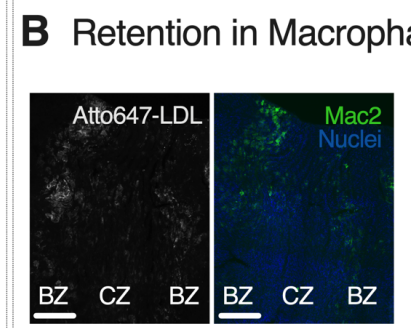

Pre-athero

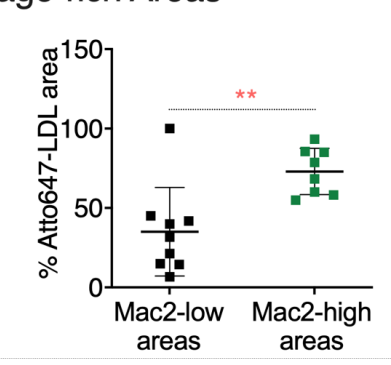

Figure 27. The border zones of the inner curvature region in pre-atherosclerotic aortas show high rates of LDL entry and retention. A. Whole-mount confocal microscopy analysis of the colocalization of labeled LDL entry (red) and retention (gray) in the inner curvature region of preatherosclerotic (left) and normal mice (right). The graphic represents aortic areas with LDL entry and retention, showing significantly higher overlap between entry and retention in pre-atherosclerotic aortas $(n=9)$ than in normal aortas $(n=6)$. ${ }^{*}<0.05$ (Mann-Whitney test). Scale bars, $500 \mu \mathrm{m}$. B, Post-staining for macrophages $(\mathrm{Mac2}+)$ showed that the scattered macrophages at this stage clustered visually in the border zones and that macrophage-rich regions had higher rates of LDL retention compared with macrophage-low areas. Bars show mean \pm SD. ${ }^{* *} \mathrm{P}<0.01$ (Mann-Whitney test). Scale bars, $500 \mu \mathrm{m}$.

The combined data thus suggest that during the pre-atherosclerotic phase, the high level of incoming LDL saturates retention in the central inner zone of the aortic arch, whereas entry and retention continue unimpeded in the border zones where the first macrophages also accumulate. 


\subsection{Saturation of LDL retention is intrinsic to the arterial wall}

The reduction in the retention of fluorescent LDL in the central inner zone of pre-atherosclerotic aortas indicated saturation of LDL retention mechanisms, which could occur by several mechanisms. First, it could be caused by simple competition from the high levels of LDL in the plasma of pre-atherosclerotic mice, similar to classic experiments where saturable binding of a labeled protein is revealed by outcompeting it with unlabeled protein. Second, it could be due to intrinsic properties of the preatherosclerotic artery wall, such as exhaustion of remaining binding sites for LDL in the extracellular matrix after weeks of LDL entry, changes in the extracellular matrix, or other cellular regulation of retention mechanisms.

To distinguish between these two possibilities, we first assessed retention shortly after the induction of hypercholesterolemia. If saturation was caused by simple competition of unlabeled LDL, induction of hypercholesterolemia itself should reduce retention of injected labeled LDL. Groups of mice were administered rAAV-PCSK9, started on the high-cholesterol diet, and given a single injection of $A p o B$ or Ctrl ASO. After 3 days with high or normal LDL levels, both groups received injections of the same dose of Atto647-LDL, and retention was analyzed by whole-mount confocal microscopy after 18 hours (Figure 28).

A Experimental Design

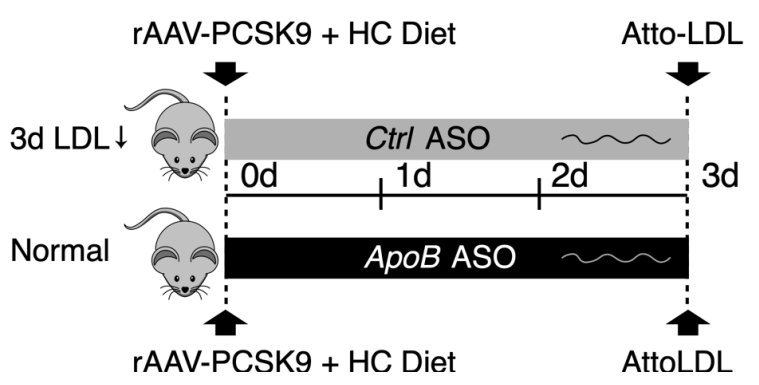

B Plasma LDL Cholesterol

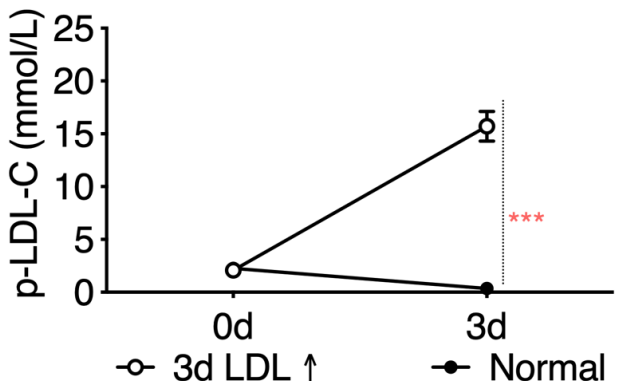

Figure 28. Effect of short-term hypercholesterolemia on LDL retention. A, Experimental design. Groups of mice were injected with rAAV-PCSK9, fed a high-cholesterol $(\mathrm{HC})$ diet, and given a single injection of either Ctrl ASO ( $3 d \mathrm{LDL} \uparrow, n=5$ ) or $A p o B$ ASO (Normal, n=5). LDL retention assays were performed after 3 days. B, Plasma LDL cholesterol concentration (mmol/L). ${ }^{* * *} \mathrm{P}<0.001$ (Mann-Whitney test at 3d).

The experiment was done under the assumption that short-term hypercholesterolemia (3 days) does not induce important changes in the capacity of LDL retention in the artery wall, therefore allowing to test solely the competition 
between injected labeled LDL and high levels of endogenous unlabeled LDL for the intimal LDL binding sites in the arterial wall.

We found that the LDL retention pattern was uniform throughout the inner curvature region (central inner and border zones) in both groups, indicating that an increase in circulating LDL alone is not sufficient to cause saturation of LDL retention in the central inner zone (Figure 29).

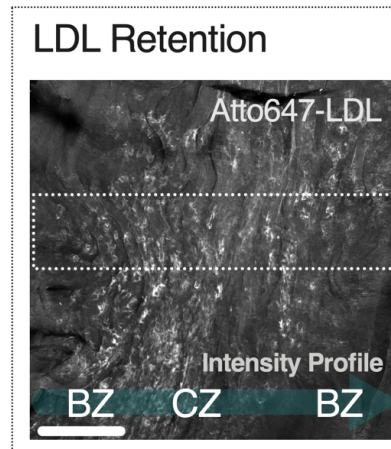

3d LDL $\uparrow$

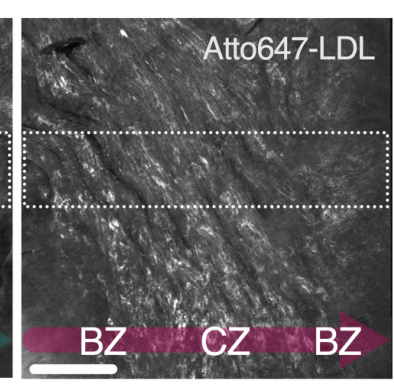

Normal

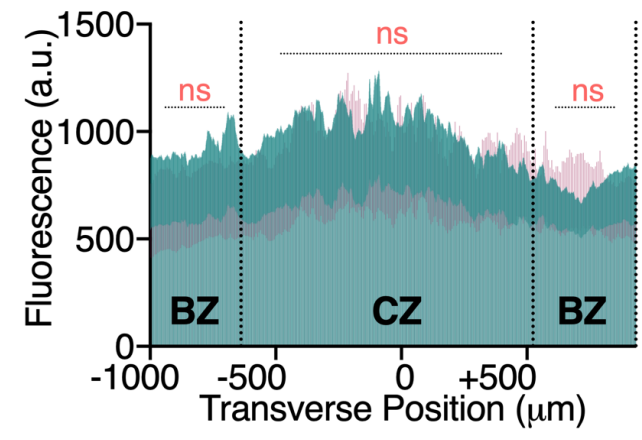

3d LDL $\uparrow$

Normal

Figure 29. LDL retention after 3 days of hypercholesterolemia. Whole-mount confocal microscopy analysis of the inner curvature region of the aortic arch. Intensity profiles across the indicated thick line show uniform retention of Atto647-LDL across the central inner (CZ) and border zones (BZs) in 3-day hypercholesterolemic and normal aortas. Graphs show mean \pm SD. ns, non-significant for AUC (MannWhitney test). Scale bars $500 \mu \mathrm{m}$.

In a second approach, we tested whether established saturation was quickly reversed after rapid LDL lowering. If saturation was caused by simple competition of labeled LDL retention from unlabeled LDL in plasma, reversal of hypercholesterolemia itself should increase retention of labeled LDL. To investigate this, we repeated our standard experiment with hypercholesterolemic and normocholesterolemic mice, but lowered LDL cholesterol in the hypercholesterolemic group with a single high dose of ApoB ASO (10 mg/kg) 3 days before analyzing Atto680-labeled LDL retention at 3.5 weeks (Figure 30A). The final plasma LDL concentration was similar in the two groups (Figure 30B). This allowed us to test the rate of retention of labeled LDL in preatherosclerotic and normal arteries in the absence of competition from high levels of endogenous unlabeled LDL. Notably, the experiment was done under the assumption that 3 weeks of sustained hypercholesterolemia (compared with 3.5 weeks in previous experiments) was already sufficient to induce the pre-atherosclerotic changes in the artery and that 3 days of LDL lowering was not sufficient to reverse them. 
A Experimental Design

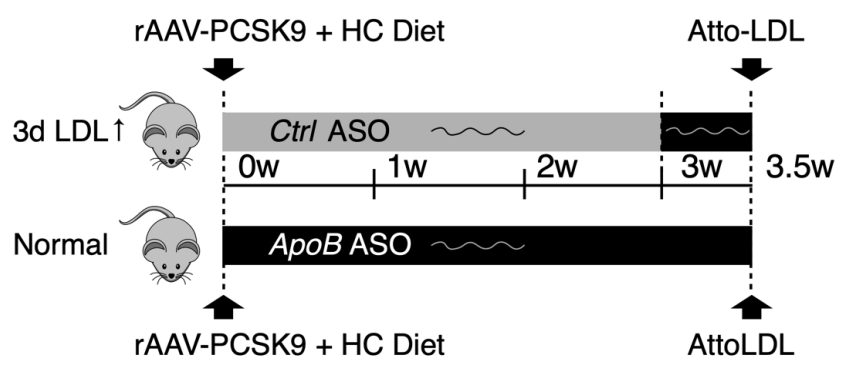

B Plasma LDL Cholesterol

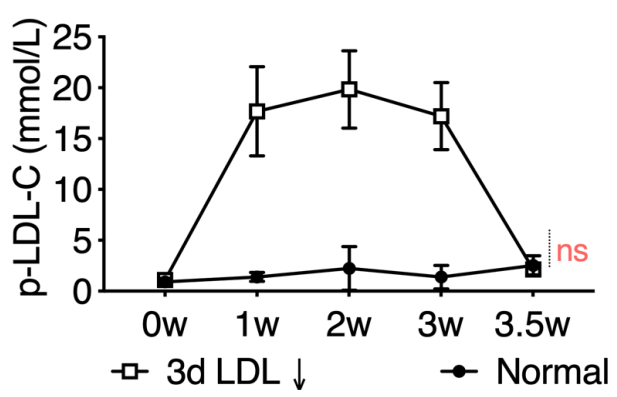

Figure 30. Effect of rapid hypercholesterolemia reversal on LDL retention. A, Experimental design. Two groups of mice were injected with rAAV-PCSK9 and started on the HC diet. One group received weekly $A p o B$ ASO injections to maintain normal plasma LDL $(n=9$, Normal) and was analyzed for LDL retention at 3.5 weeks. The second group received weekly Ctrl ASO injections but was given a single high dose $(10 \mathrm{mg} / \mathrm{kg})$ injection of $A p o B$ ASO 3 days before the endpoint to bring plasma LDL levels back down to normal $(n=5,3 d$ LDL $\downarrow$ ). B. Plasma LDL cholesterol concentration (mmol/L) curves showing comparable LDL levels between the two groups at endpoint. ns, non-significant (Mann-Whitney test at $3.5 \mathrm{w})$. Bars show mean \pm SD.

The intensity profile analysis showed that although competition from endogenous LDLs was removed, labeled LDL retention saturation was maintained in the central zone of the inner curvature region (Figure 31).

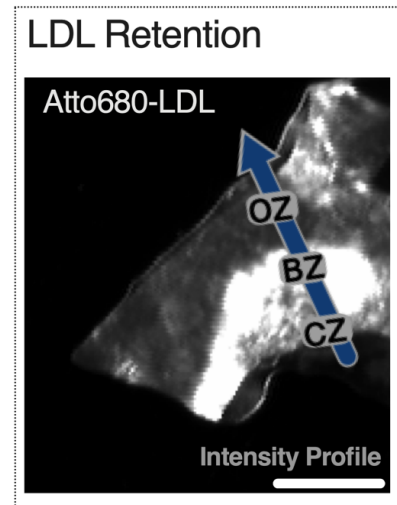

3d LDL $\downarrow$

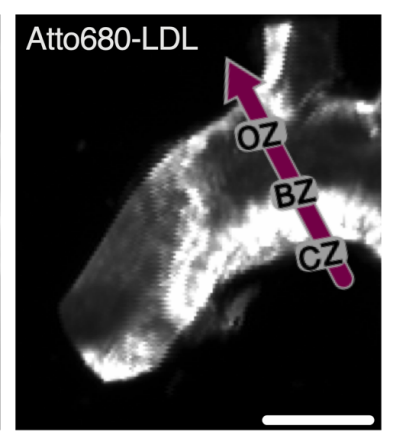

Normal

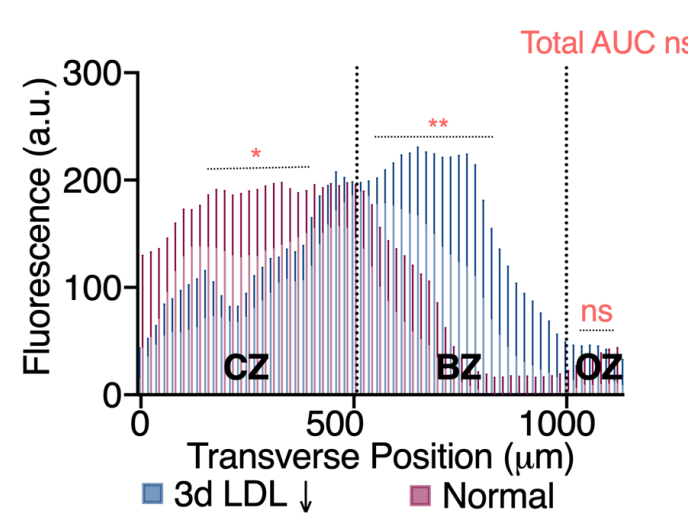

Transverse Position $(\mu \mathrm{m})$
3d LDL $\downarrow \quad \square$ Normal

Figure 31. LDL retention after 3 days of LDL lowering. En face analysis (inverted grayscale) of Atto680-LDL retention. Intensity profiles along the indicated direction show persistent saturation of LDL retention in the central inner zone of $3 d$ LDL $\downarrow$ mice, whereas retention in the border zone was increased. Graphs show mean $\pm S D$. ns, non-significant, ${ }^{*} P<0.05,{ }^{* *} P<0.01$ for AUC (Mann-Whitney test). Scale bars, $1 \mathrm{~mm}$.

This indicates that the reduced retention of labeled LDL in hypercholesterolemic mice involved changes intrinsic to the arterial wall and was not explained by simple 
competition from circulating unlabeled LDL. In the border zone, retention of labeled LDL in the pre-atherosclerotic arterial wall was increased when competition from endogenous LDLs was removed. This may suggest that the capacity for retention in the border zone is increased compared with the normal artery, but that this is masked when analyzed in hypercholesterolemic mice because of competition from endogenous unlabeled LDL.

Combined, the results obtained from the two experimental approaches suggest that the reduced retention of labeled LDL observed in the central zone of the inner curvature region is not caused by the difference in plasma LDL levels between hypercholesterolemic and normocholesterolemic mice, but by saturation of binding sites or regulation of other retention mechanisms in this zone of the ascending aortic arch.

\subsection{LDL entry and retention rates predict the course of plaque development}

To test whether the rates of LDL entry and retention in the pre-atherosclerotic wall predict the subsequent development of atherosclerosis, we injected mice with rAAV-PCSK9 and fed them the high-cholesterol diet for 0, 4, 6, 12, or 24 weeks (Figure 32). We then evaluated how the topography of the atherosclerotic lesions related to the topography of LDL entry and retention distribution across the aortic arch.

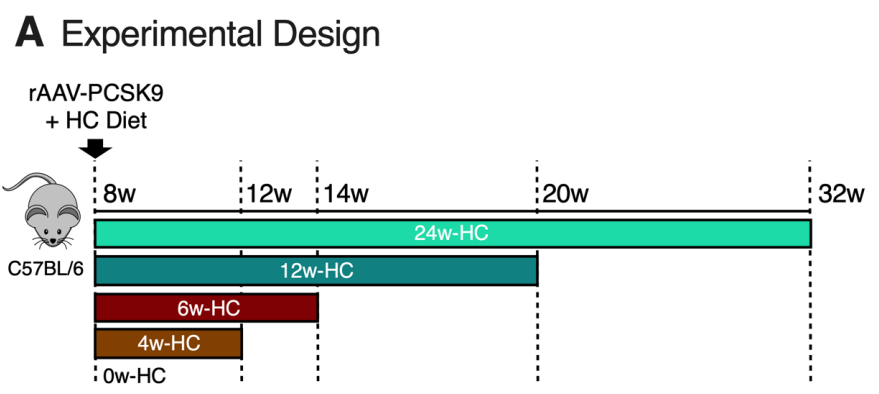

B Plasma LDLCholesterol

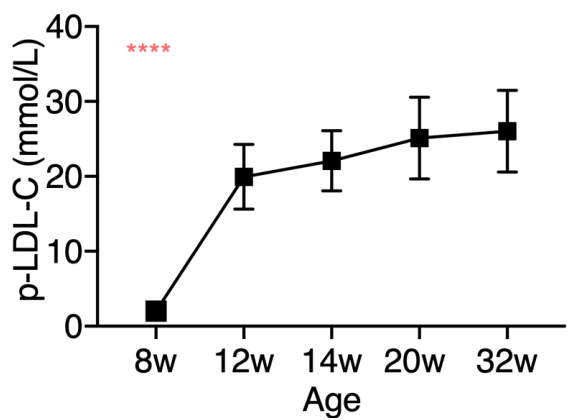

Figure 32. Correlation between the topography of LDL entry and retention and topography of atherosclerotic lesions. A, Experimental design. Groups of 8-week-old mice were injected with rAAVPCSK9 and fed a high cholesterol (HC) diet for $0(n=4), 4(n=6), 6(n=7), 12(n=7)$, or 24 weeks $(n=6)$. B, Plasma LDL cholesterol concentration (mmol/L) at $8,12,14,20$, and 32 weeks of age. ${ }^{* * * *} P<0.0001$ (Kruskal-Wallis test). 
Plaque formation was first evident at 6 weeks at the border zones of the inner curvature region. At 12 weeks of atherogenesis, plaques started to incorporate the central zone of the inner curvature region until completely covering it at 24 weeks. The outer zone did not develop atherosclerosis over the time period studied (Figure 33).
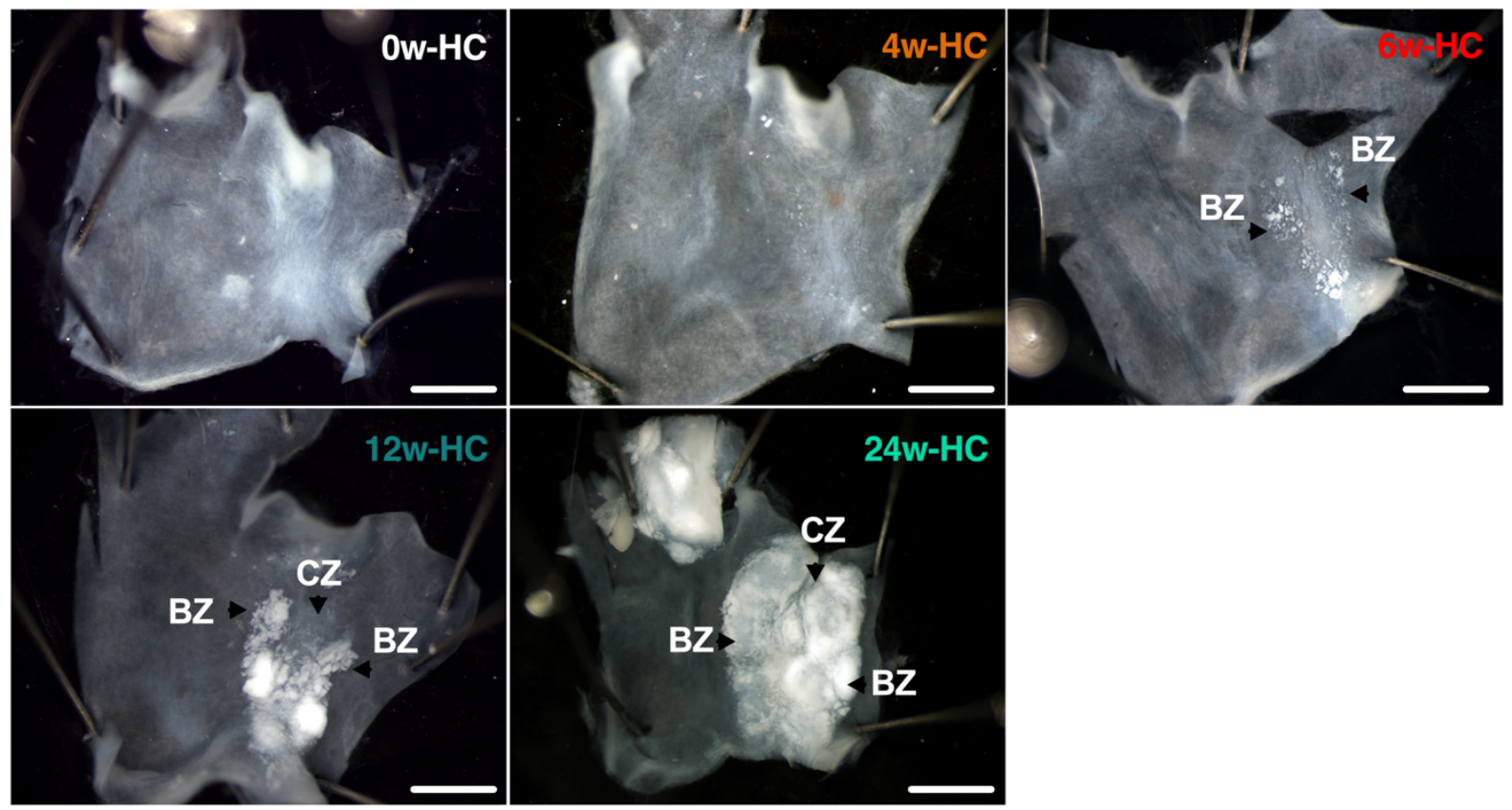

Figure 33. LDL entry and retention rates in the pre-atherosclerotic wall predict how atherosclerosis develops. Representative images of the inner aortic arch examined with a Nikon stereomicroscope. Images show plaque formation starting at the border zones of the inner curvature region after $6 w$ of hypercholesterolemia $(n=7)$, developing toward the central zone after $12 w(n=7)$ until becoming uniform across the inner arch after $24 w(n=6)$. Border and central inner zones with atherosclerotic lesions are indicated with black arrows. Scale bars, $1 \mathrm{~mm}$.

\subsection{Atherogenesis is associated with loss of saturation of LDL retention mechanisms}

To evaluate whether the saturation of LDL retention observed in the central inner zone of the pre-atherosclerotic aorta persists in lesioned arteries, we induced atherosclerosis in two groups of mice through rAAV-PCSK9 injection and highcholesterol diet for 12 weeks. In one group, one week prior to the endpoint, we rapidly reduced plasma LDL by single injection of high dose ApoB ASO (10 mg/kg); the other group received a single high dose of Ctrl ASO (Figure 34A-B).

The experiment was done under the assumption that one week of 
normocholesterolemia is not sufficient to induce changes in the biology of atherosclerosis (i.e. content of macrophages, foam cells, ECM composition, and gene expression changes of key cytokines and scavenger receptors), therefore allowing to test only the effect of different endogenous plasma LDL levels on the labeled LDL retention capacity of the atherosclerotic artery wall.

A Experimental Design

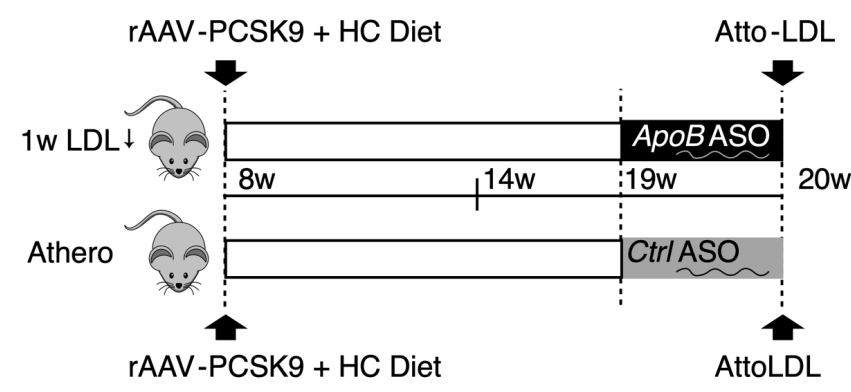

B Plasma LDL Cholesterol

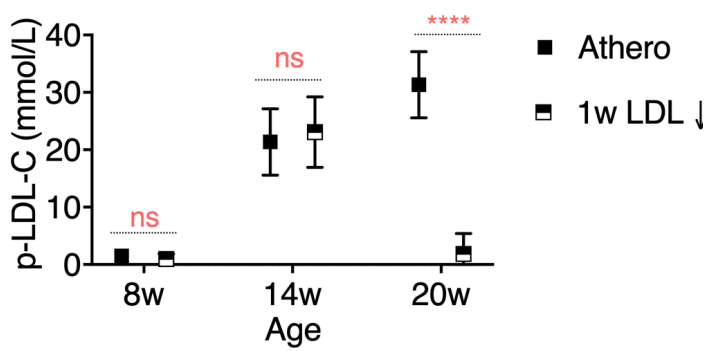

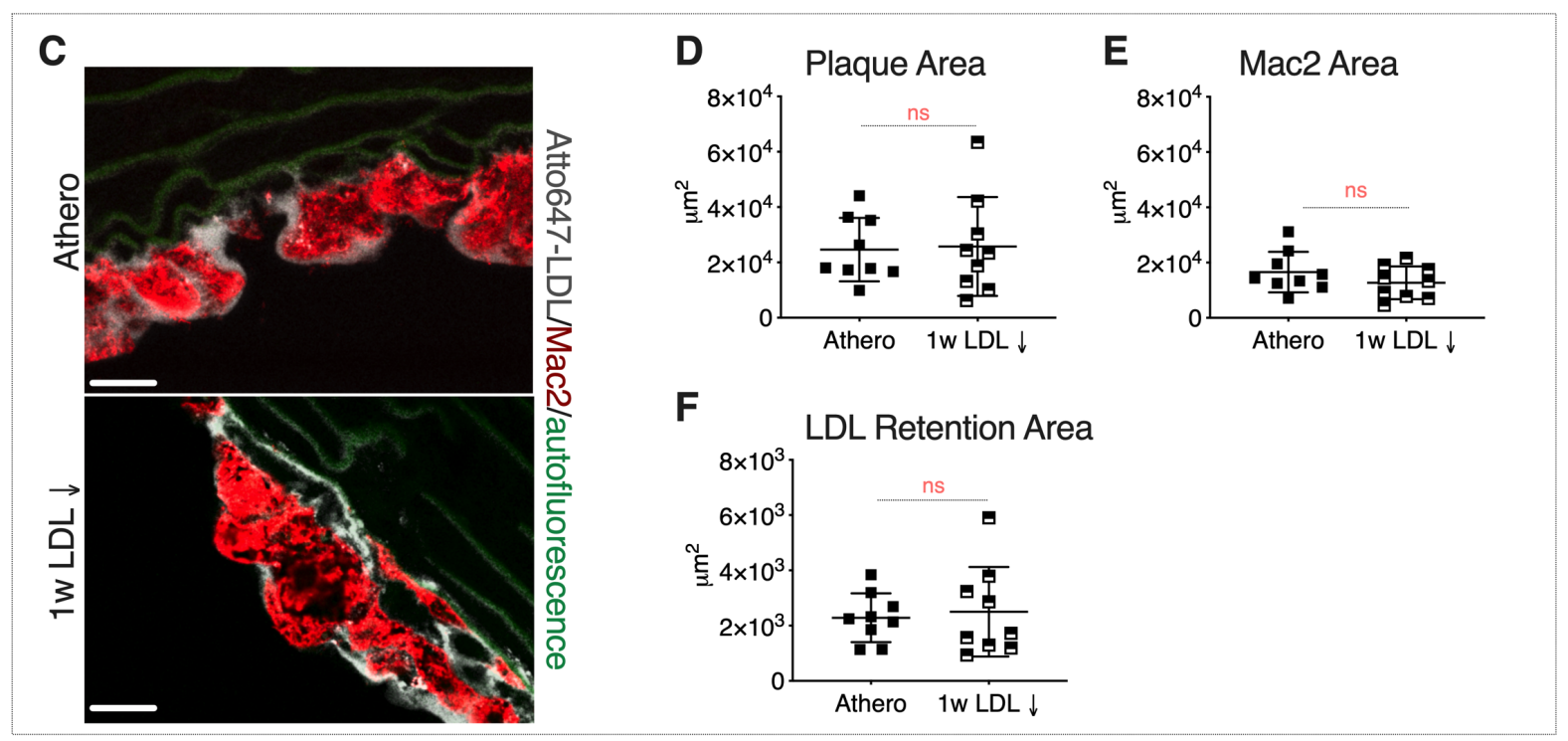

Figure 34. Atherogenesis is associated with loss of saturation of LDL retention mechanisms. A, Experimental design. 8 weeks old wild-type mice were injected with rAAV-PCSK9 and fed a high cholesterol (HC) diet for 12 weeks. One week before the endpoint, one group of mice received a high dose injection of $A p o B$ ASO (10 mg/kg) (1w LDL $\downarrow, n=9)$ to lower LDL cholesterol. Another group received a single high dose of Ctrl ASO (Athero, $n=9$ ). B. Plasma LDL cholesterol concentration in the two groups after 0,6 , and 12 weeks. ns, non-significant, ${ }^{* * *} \mathrm{P}<0.0001$ (Mann-Whitney test at each time point). C, Representative aortic arch cross sections from Athero $(n=9)$ and $1 w \operatorname{LDL} \downarrow(n=9)$ mice, showing Atto647-LDL retention (gray), macrophage content (red), and elastin autofluorescence (green). Scale bars, $20 \mu \mathrm{m}$. D-F, Quantification of plaque and macrophage area, showing no significant difference between the two groups (Mann-Whitney test). D, Quantification of Atto647-LDL retention area, showing that retention is not saturated by the high plasma LDL concentration in the Athero group. ns, non-significant (Mann-Whitney test). 
At 18 hours after injection of Atto647-LDL, we analyzed aortic arch cross sections by confocal microscopy. As expected, plaque size and macrophage content were not changed by the short period of normal LDL levels. Notably, retention of Atto647-LDL was also similar in both groups, suggesting that once the atherosclerosis process starts, the saturation of LDL retention mechanisms observed during the preatherosclerotic phase is lost (Figure 34C-F). 




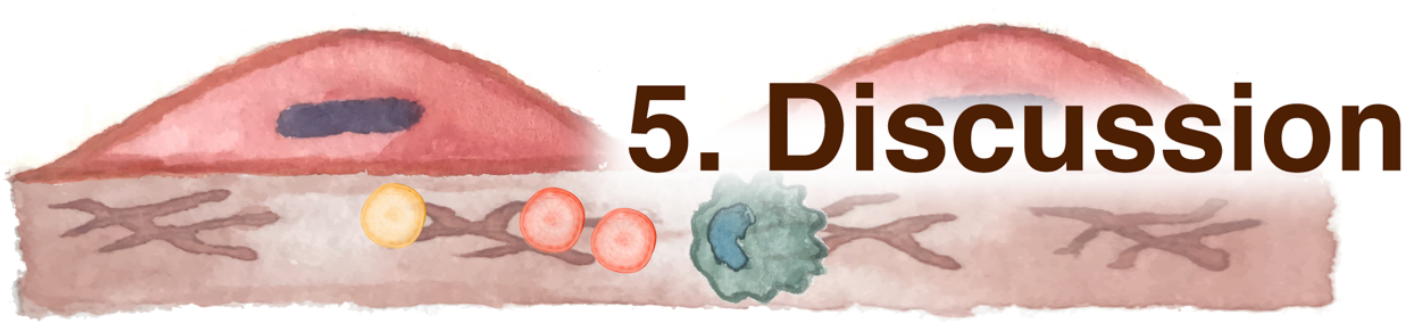


Atherosclerosis is a slowly progressing disease that starts in young adults but remains harmless for decades. It is an extremely widespread pathology and even individuals that never suffer symptoms from atherosclerotic disease and die from other causes often have considerable atherosclerotic lesion formation. In those individuals, atherogenesis was just not fast enough to lead to symptoms before death by other causes. Indeed, the goal for prevention is not so much to avoid atherosclerosis but to postpone the age of clinical symptom debut. In this context, understanding the initial stages of the disease has high importance as this could help provide tools to retard the time by which lesions are initiated and spread across the arterial tree and thereby result in a delay in life-threatening atherothrombotic complications.

The focal pattern of lesion development is a striking feature of atherogenesis and studying the topography of atherosclerosis is an important inroad to understanding what leads to the onset of the disease and how it can be delayed. Lesions develop initially near branch sites and on the inside of arterial curvatures, while other regions take much longer to be affected and some are completely protected. There is little doubt that it is the selective accumulation of LDL at the most atherosclerosis-prone sites that causes the first lesion formation, but why LDL accumulates there and not elsewhere remains uncertain. ${ }^{11}$

\subsection{Functional studies reveal the importance of both LDL entry and LDL retention processes}

Both LDL entry and LDL retention are needed for LDL accumulation and as would be expected, functional studies show that blocking steps in either process can lead to a reduction in LDL accumulation and retardation of lesion formation. An overview of some of the interventions that have been studied and their effects is given in Figure 35.

LDL entry can be inhibited by knockout of Caveolin 1, which is an important constituent of caveolae that carry LDL transcytosis through intimal endothelial cells. Lack of Cav1 causes reduced LDL entry and less atherosclerosis formation in mice. ${ }^{36,38}$ Also, knockout of the gene encoding SR-B1 in $\mathrm{Apoe}^{-/-}$or $\mathrm{Ldlr}^{-/-}$mice reduces LDL transcytosis and results in reduced atherosclerotic lesions compared to controls despite no change in circulating LDL levels. ${ }^{32}$ Notably, an earlier study 
showed that the endothelium-specific over-expression of human SR-B1 also decreases atherosclerosis in $A p o e^{-/-}$mice, suggesting that SR-B1 may play other roles in endothelium outside LDL transcytosis. ${ }^{133}$

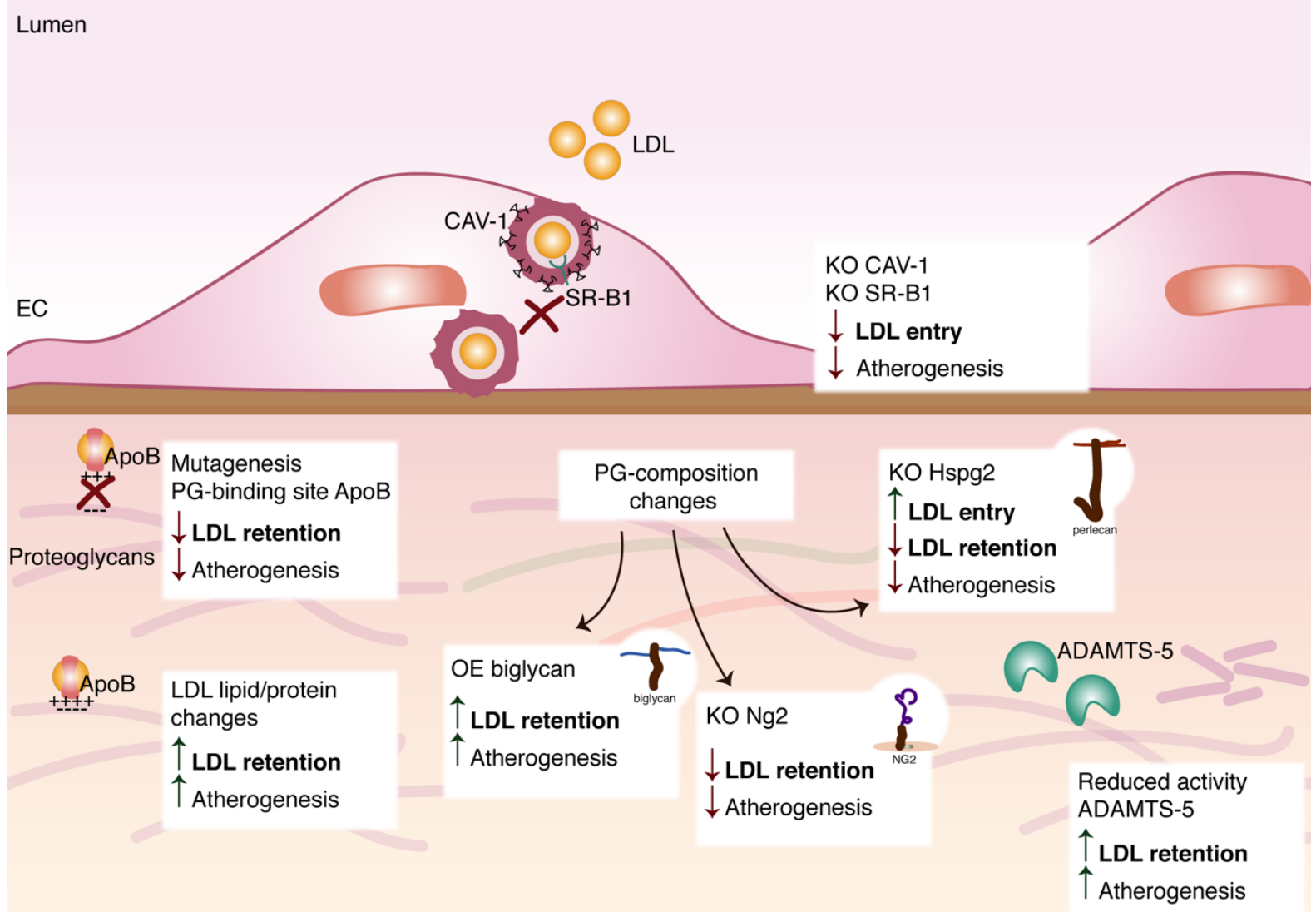

Sub-endothelial space

Figure 35. Schematic representation of the functional studies showing LDL entry and LDL retention as necessary steps for atherosclerosis initiation. Details are discussed in the main text. $\mathrm{EC}=$ Endothelial cell; $\mathrm{PG}=$ Proteoglycans.

Specific attenuation of the ability of LDLs to be retained in arteries has also been achieved in experimental models and has been found to delay atherosclerosis initiation. Skålén et al. showed that mutagenesis of the primary proteoglycan-binding site in $A p o B$ results in reduced atherosclerosis in mice, independently of the effects on plasma LDL levels. ${ }^{134}$ Several other changes in the lipid and protein composition of LDL that result in increased affinity for proteoglycans have been shown to promote atherogenesis. ${ }^{47,51,135}$ In addition, changes in the arterial composition of LDL-binding proteoglycans affect the susceptibility to atherosclerosis. For example, $\mathrm{Ldll}^{-1-}$ mice overexpressing biglycan showed increased atherosclerosis development that correlated with vascular biglycan content. ${ }^{136}$ Biglycan deficiency did not exert the 
expected protective role against the development of atherosclerosis in the same model. Yet, these mice showed increased vascular perlecan content, suggesting a response of the vasculature to compensate for the biglycan deficiency. ${ }^{137}$ Another example is given by Apoe ${ }^{-/-}$mice lacking the proteoglycan neural/glia antigen 2 (NG2), able to bind LDL through hydrophobic interactions. Double knockout mice $\left(\mathrm{Ng}^{-/-} / \mathrm{Apoe}^{-/-}\right)$show reduced $\mathrm{LDL}$ retention and develop less atherosclerosis compared to their Apoe ${ }^{-/-}$controls. ${ }^{138}$ Other manipulations of the arterial matrix that alter the content of LDL-binding proteoglycans also affect atherosclerosis initiation. ${ }^{139,140}$ For instance, the metalloprotease A disintegrin and metalloproteinase with thrombospondin motifs 5 (ADAMTS-5) is a major proteoglycan-degrading enzyme. Didangelos et al. showed that reduced activity of ADAMTS-5 results in proteoglycan accumulation, increased LDL retention in the artery wall, and increased atherosclerosis. ${ }^{139}$ Moreover, the handling of arterial-wall enzymes that promote LDL retention also influences atherosclerosis. ${ }^{128,141,142}$

Finally, an informative study from Tran-Lundmark et al. allowed the separation of LDL entry and LDL retention by creating a genetic model with opposite effects on the two processes. They generated heparan sulfate (HS)-deficient perlecan $\left(H s p g 2^{\Delta 3 / \Delta 3}\right)$ mice with an $A p o e^{-/-}$background. Perlecan is involved in LDL retention but also contributes to the endothelial barrier of the arterial wall. $\mathrm{Hspg}^{\Delta 3 / \Delta 3} \backslash A p o e^{-/-}$ mice therefore exhibit increased LDL entry but lower LDL retention in the artery wall. They developed significantly reduced atherosclerotic lesions compared to $A p o e^{-/-}$ controls, suggesting a key role for LDL retention in atherogenesis, regardless of the LDL entry levels. ${ }^{11,143}$ Furthermore, experimental manipulation of blood flow that increases atherosclerosis susceptibility in mouse carotids increased LDL retention but not LDL entry. ${ }^{72}$

These functional studies highlight the fact that both LDL entry and LDL retention are necessary steps for LDL accumulation and atherogenesis, but they do not speak to which is the rate-limiting mechanism during the natural history of atherosclerosis.

\subsection{Which process determines atherosclerosis susceptibility?}

Various studies have implicated either increased LDL entry or increased intimal LDL retention as the distinguishing feature of susceptible arterial regions. Early studies 
in rabbits concluded that the entry of radiolabeled LDL into aortic segments predicted atherosclerosis development, ${ }^{26}$ but others found differing LDL accumulation in regions with similar LDL entry rates, indicating that selective LDL retention was the rate-limiting step. ${ }^{39}$ Therefore, results in this field have not been consistent, and it is clear that further investigation is needed of the association between topographical differences in LDL entry and retention and atherosclerosis susceptibility.

The present study delivers a systematic analysis of LDL dynamics in the mouse aortic arch during early atherogenesis. We report entry and retention rates across susceptible and resistant regions and contrast them between normal arteries and arteries on their way to initiating atherosclerosis. This approach goes beyond previous analyses by providing higher resolution than achievable with radiolabeling and by using tools that allowed us to compare mice with high and normal plasma LDL without creating differences in the clearance of the labeled LDL probe. Using these tools, we obtained insights into how LDL entry and retention in the arterial wall predict the onset and progression of atherosclerotic lesions in the mouse. Our main findings are discussed in detail in the next sections.

\subsection{Rates of LDL entry and retention define regions with differing atherosclerosis susceptibility}

The first main finding of the thesis was that differences in LDL entry and retention rates in the normal (non-atherosclerotic) mouse aortic arch divide it into three regions with differing atherosclerosis susceptibility. The atherosclerosis-resistant zone (the outer part of the arch) takes up and retains little LDL and develops no atherosclerosis over 24 weeks of high plasma LDL. The intermediate-susceptibility zone in the central part of the inner arch retains LDL but does not have a high average rate of LDL entry; this zone is initially unaffected by atherosclerosis but develops it over 6-24 weeks. Finally, the highly susceptible border zones that lie between the resistant and intermediate-susceptibility regions on the lower ventral and dorsal walls of the aortic arch; these zones have high rates of both LDL entry and retention and are the location where lesions are initiated.

These observations offer a unifying explanation for previous studies that have variably associated subsequent atherosclerosis development with the pattern of either 
LDL entry or retention. We find that both LDL entry and retention are associated with atherosclerosis susceptibility but with different time horizons. The distribution of LDL entry in the normal artery predicts sites of imminent atherogenesis, whereas the distribution of retention predicts the surface area that will eventually be covered with lesions.

Why these regions show different properties requires further investigation. Possibly, an important role could be played by the wall shear stress generated by the blood flow in the different regions. The outer zone of the aortic arch (atherosclerosisresistant region) is subjected to high laminar shear stress, which is atheroprotective. ${ }^{144-146}$ Areas of high laminar shear stress, in which endothelial cells are elongated, aligned, and protected from inflammation, ${ }^{74}$ have already been correlated with low rates of LDL entry and retention. ${ }^{26,39,70,117}$ Therefore, this part of our results confirms previous studies. Conversely, the inner aortic curvature region composed of both the central inner zone (intermediate-susceptibility region) and the border zones (highly-susceptibility region), is well known to be subjected to low wall shear stress. ${ }^{144-}$ 146 Although this area has traditionally been referred to as homogeneously atherosclerosis-prone, this study shows the existence of site-specific dissimilarities. Therefore, there could be unknown additional regional differences within the inner curvature area, with the wall shear stress being lower and more disturbed at the border zones compared to the central inner zone.

As discussed in the next sections, disturbed flow induces several changes in the arterial intima that could affect LDL entry, LDL retention, and inflammatory activation which in turn could play a key role in altering atherosclerosis-susceptibility.

First, low shear stress increases endothelial permeability, which together with increased residence time of circulating LDL characteristic of flow stagnation areas, could increase rates of LDL entry. ${ }^{69,147}$ Local hemodynamics could also affect LDL entry by altering the thickness of the endothelial glycocalyx, a thick layer of glycoproteins and heparan sulfate (HS), hyaluronic acid (HA), and chondroitin sulfate (CS) proteoglycans that lines the endothelial surface and extends from the endothelium towards the lumen. ${ }^{148}$ The glycocalyx is an important barrier to solutes and is involved in the regulation of LDL transport into the arterial wall, although the exact nature of the interactions between LDL and glycocalyx is not well understood. ${ }^{149}$ The extent of the glycocalyx depends on both synthesis and endothelial apoptosis rates. ${ }^{149}$ High shear stress has been shown to reduce the rate of endothelial cell 
apoptosis $^{150,151}$ and to increase endothelial cell synthesis of glycocalyx components. ${ }^{152,153}$ This is consistent with the observation that the glycocalyx is highest at atherosclerosis-resistant sites and thinnest at atherosclerosis-prone sites. Upon cholesterol challenge, it is reduced in all the arterial zones. ${ }^{154,155}$

Second, lowered wall shear stress or accompanying differences in tensile stress of the arterial wall could influence local vascular smooth muscle cells (VSMCs) to modulate and secrete LDL-binding matrix components, including proteoglycans. ${ }^{23}$ Notably, the introduction of disturbed laminar flow in atherosclerosis-resistant arterial segments was shown to be sufficient to increase LDL retention. ${ }^{72}$ In the present study, LDL retention appeared uniform across the whole inner curvature region (border zones and central inner zone) in the normal mouse aortic arch. However, it is still possible that differential blood flow profiles within the inner curvature region may favor LDLbinding intimal matrix synthesis in the border zones compared to the central inner zone. This increased capacity may only become visible once the available binding sites begin to be saturated by sustained hypercholesterolemia and not under normocholesterolemic conditions because of the overabundance of free binding sites.

Third, altered wall shear stress may prime the endothelium to respond to proatherogenic stimuli by enhancing inflammation. ${ }^{14,146,156}$ High laminar shear stress suppresses pro-inflammatory activation and leukocyte recruitment, ${ }^{157,158}$ while low sheer stress promotes a pro-inflammatory state with activation of pro-inflammatory signaling pathways (i.e. NF-kB signaling pathway). ${ }^{146}$ This pathway, in turn, activates genes implicated in atherosclerosis, such as genes encoding adhesion molecules (i.e. VCAM-1 and ICAM-1) ${ }^{159}$ and chemoattractants (i.e. MCP-1) ${ }^{160}$ that stimulate the recruitment of inflammatory cells into the sub-endothelial space. Studies of these pathways have generally considered the inner curvature region as a whole and future studies are needed to explore whether pro-inflammatory signaling is particularly abundant in the border zones. Consistent with that idea, we found that initial macrophages accumulated mostly at the border zones, which could potentially increase the capacity for LDL retention at these sites due to increased cellular uptake and release of pro-inflammatory cytokines stimulating extracellular matrix synthesis. We cannot, however, determine in the present study whether macrophages were recruited to the border zones because of the special LDL handling processes at these sites or vice versa. 
To explore whether flow-controlled mechanisms could explain the increased atherosclerosis susceptibility of the border zones, it would first be important to evaluate whether regional differences in wall shear stress exist between the border zones and the central inner zone of the inner curvature region. To do so, an important step would be to perform careful measurements of the geometries followed by blood flow modeling by computational fluid dynamics, directing special attention to the heterogeneity of the inner curvature area. Moreover, it would be interesting to map glycocalyx thickness, components of endothelial junctions, proteoglycan composition and differential expression of LDL transport elements (i.e. caveolae, CAV-1, SR-B1, ALK-1). This will require additional immunostaining, bulk or scRNA-seq gene expression profiling in micro-dissected tissues, and transmission electron microscopy (TEM). If promising associations are seen, intervention studies, e.g. enzymatic glycocalyx reduction or gene knockouts, could be performed to analyze the causal importance for the differences in atherosclerosis susceptibility across the aortic arch.

\subsection{The atherosclerosis-intermediate susceptibility region has saturable LDL retention}

The second main finding emerging from our analysis is that the intermediate susceptibility of the central zone of the inner curvature region is associated with saturation of LDL retention. This was evident from two experimental outcomes. First, global LDL entry rates in the aortic arch increased during the pre-atherosclerotic phase, but this was not mirrored by similar increases in the global rate of LDL retention. Second, pre-atherosclerotic arteries in hypercholesterolemic mice retained less total labeled LDL than arteries in normocholesterolemic mice, and this was explained by reduced retention in the central zone of inner curvature.

The saturation of LDL retention mechanisms in the central inner zone was not due to competition from the high plasma concentration of endogenous LDL in the hypercholesterolemic mice, since it did not develop immediately after induction of hypercholesterolemia and did not disappear after a short period of normocholesterolemia. The first experiment was done under the assumption that 3 days of hypercholesterolemia do not induce important changes in the artery wall, therefore allowing to test only the competition from high levels of endogenous LDL for 
intimal LDL binding sites. To our knowledge, the impact of such short-term hypercholesterolemia on arterial wall composition has not been directly tested, but we find it reasonable that major changes are not induced. The second experiment was done under the assumption that 3 weeks of sustained hypercholesterolemia are sufficient to induce the pre-atherosclerotic changes in the artery and that 3 days of LDL lowering is not sufficient to reverse them. Although quantitative analysis has not been done, visual inspection of the en face scans from mice during the first weeks of hypercholesterolemia suggests higher LDL entry at the border zones and lower LDL retention in the central inner zone already at 2 weeks, supporting this hypothesis. Llodrà et al., showed increased arterial clearance of monocytes to lymphoid organs from aortic lesions exposed to 3 days of normocholesterolemia by aortic transplantation from atherosclerotic $L d l r^{-/-}$mice into wild-type mice. ${ }^{161}$ Using this model, the authors also observed plaque size reduction after 3 days, which, however, was not observed by Bartels et al. after 1 week of LDL lowering with ApoB ASO treatment in atherosclerotic Ldlr ${ }^{-/-}$mice. ${ }^{117}$ To our knowledge, short-term hypercholesterolemia reversal has not been tested in pre-atherosclerotic mice, therefore we should keep awareness of the limitations of the assumption.

Notably, in the central zone of the inner curvature region there was no saturation at the level of LDL entry, which was similar between pre-atherosclerotic and normal mice. The combined observations therefore suggest that, during the preatherosclerotic phase, the intima has a limited capacity for binding LDL and that when this capacity is reached most incoming LDL returns to the circulation from the vessel wall without being retained.

It is important to keep in mind that, although hypercholesterolemic mice play a central role in the investigation of the pathogenesis of atherosclerosis, they do not recapitulate all the features of the human disease. Indeed, while early lesions in humans are characterized by adaptive intimal thickening, this is not the case in mice. ${ }^{108}$ Therefore, the saturable LDL binding capacity of the artery wall observed in pre-atherosclerotic mouse arteries might not occur in humans, where adaptive intimal thickenings can grow to be as thick as the underlying media. ${ }^{73}$ Also, heterologous (human) LDL could have different binding affinities to arterial wall receptors and intimal matrix from homologous (mouse) LDL. ${ }^{21,162}$

In order to understand why the saturation of LDL retention occurs, it would be of interest to evaluate whether it is linked to the limited capacity of the LDL binding 
sites. A logical step would be to evaluate whether LDL retention saturation in the central inner zone is maintained after induction of intimal hyperplasia ${ }^{163}$ and thereby in the presence of increased intimal tissue volume and total number of LDL binding sites. In vitro LDL-binding assays ${ }^{163}$ on frozen aortic arch cross sections from preatherosclerotic and normal mice could also give insights on the LDL binding capacity of the artery wall. Global gene expression analysis at the inner aortic curvature region in pre-atherosclerotic and normal arteries did not show differential expression of extracellular matrix proteoglycans. However, the analysis was done taking into account the whole artery wall of the inner curvature region, not allowing to differentiate between the border and central inner zones. RNA-sequencing and differential gene expression analysis after micro-dissection of the border and central zones of the inner curvature region could provide a better understanding of this phenomenon.

\subsection{The conversion from saturable to non-saturable LDL retention accompanies the onset of atherosclerosis}

The third main finding is that conversion from saturable to non-saturable LDL retention accompanies the onset of atherosclerosis. We found comparable retention of labeled LDL at sites of atherogenesis whether plasma LDL was high or was lowered shortly before analysis, including in the central zone of the inner curvature region. This indicates that the capacity for retention of incoming LDL increases substantially with initiation of atherosclerosis and that it shows no saturation even in the presence of high plasma LDL levels in hypercholesterolemic mice.

The experiment was done under the assumption that one week of normocholesterolemia is not sufficient to induce changes in the atherosclerotic artery wall. The absence of saturation for LDL retention in mature plaques is consistent with previous findings by Bartels et al., who measured an unchanged pool size of radiolabeled LDL within the arterial wall after 1 week of LDL lowering in atherosclerotic

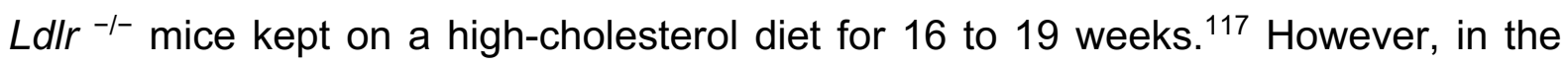
latter study they also observed an improved barrier function of the aortic endothelium towards the entry of plasma radiolabeled LDL particles after 1 week of treatment with ApoB ASO. ${ }^{117}$ Potentially, a lower LDL entry could influence our retention assay in mature plaques if LDL entry was a limiting factor for LDL intimal binding. This was not 
the case in pre-atherosclerotic animals, but the possibility cannot be excluded and further investigation is required to test this. Moreover, as mentioned in the previous section, Llodrà et al. showed increased migration of monocytes from the artery wall to lymph nodes shortly after the induction of lowered LDL plasma levels through aortic transplantation from atherosclerotic donor mice into normocholesterolemic recipient mice. ${ }^{161}$ These limitations need to be addressed.

It will be important in future studies to determine the mechanisms that convert saturate sites to non-saturable sites and the potential causal importance of this for the onset of atherogenesis. Binding of LDL to intimal proteoglycans is an important step in atherosclerosis initiation, ${ }^{134}$ and saturation of binding sites in the glycosaminoglycan-chains of intimal proteoglycans may be the mechanism underlying saturation in the intermediate-susceptible area of the central inner curvature region. It is also known that LDL retention mechanisms shift once lesions are initiated. Direct binding of LDL-ApoB to proteoglycans gradually loses importance, and other types of retention that involve the LDL lipid moiety take over, such as binding to lipoprotein lipase (LPL) ${ }^{142}$ and enzyme-catalyzed aggregation (e.g secretory sphingomyelinase (S-SMase)). ${ }^{128}$ Indeed, during the pre-atherosclerotic phase, LPL is mostly localized in the arterial media, but once lesions start to become established, it begins to be widely distributed in the intimal extracellular matrix secreted by the accumulating macrophage foam cells. ${ }^{164}$ The LPL bound to the intimal proteoglycans then promotes the retention of LDL acting as a molecular bridge, especially when LDL are extensively oxidized and therefore cannot bind directly to the glycosaminoglycan-chains because of the reduced number of positive charges. ${ }^{142}$ Similarly, secretory acid S-SMase starts to appear once lesions develop and promotes LDL retention by inducing lipoprotein aggregation and thereby enhanced uptake by macrophages and decreased efflux from the artery wall of the large LDL aggregates. ${ }^{128}$ Combined, these mechanisms may have a higher capacity to retain LDL in the intima than LDL-ApoB-proteoglycan binding alone. Also, potentially important is the increase in intimal distribution volume for incoming LDL in plaques compared with non-diseased arteries.

Understanding how LDL retention shifts from a low-capacity, saturable process to a high-capacity, non-saturable process during the initiation of atherosclerosis is an important future goal. 


\subsection{Synthesis of findings in a model}

Overall, our combined results lead us to propose the model shown in Figure 36 for the processes governing LDL accumulation and atherosclerosis onset in the inner curvature region of the mouse aortic arch.

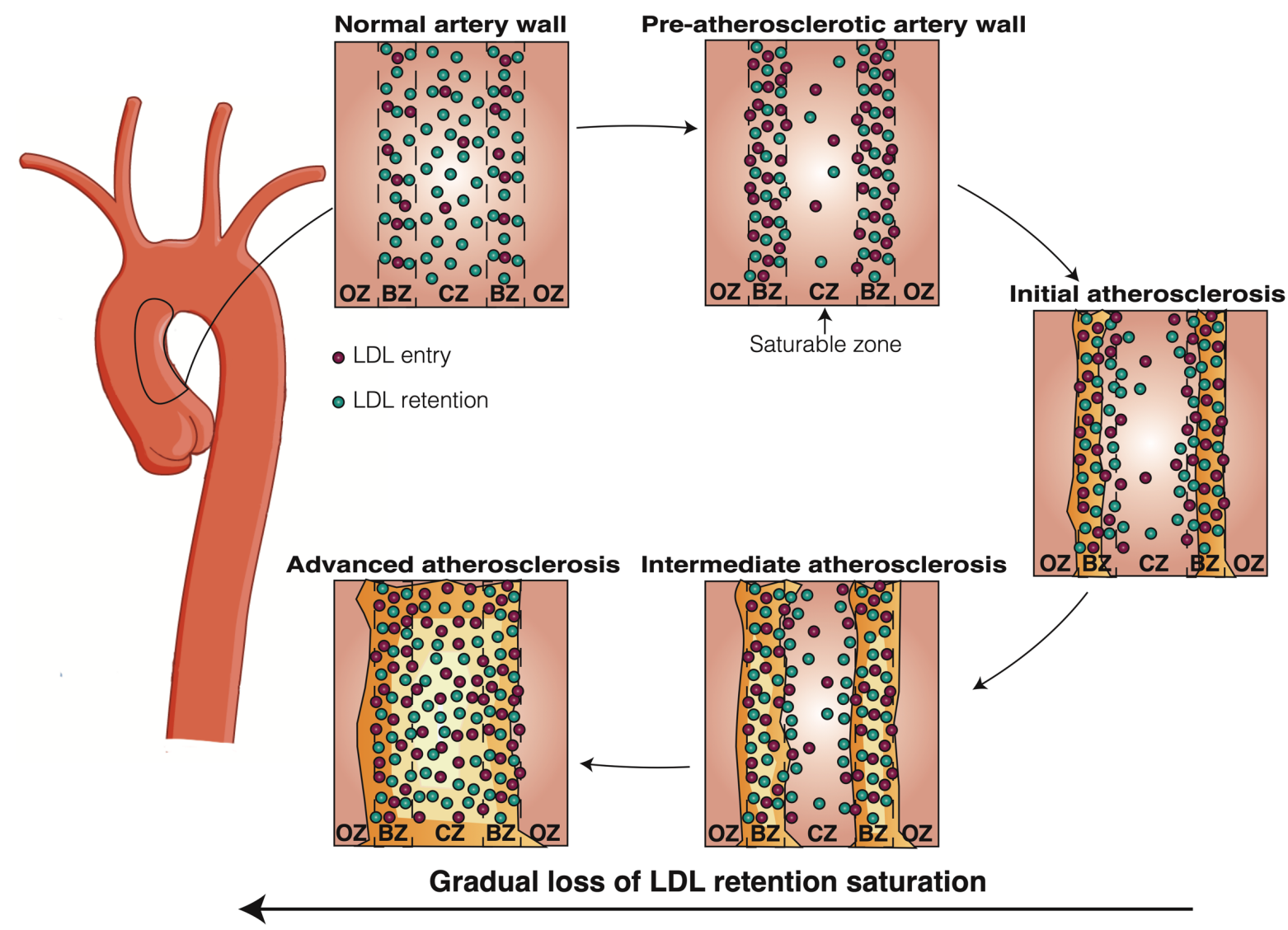

Figure 36. Model depicting the dynamics of LDL entry and retention in the aortic arch of normal, pre-atherosclerotic, and lesioned arteries. OZ= Outer zone; BZ= Border zone; CZ=central zone. The $\mathrm{OZ}$ has low LDL entry (red) and retention (green) and does not develop atherosclerosis. The BZs have high LDL entry and retention and are the sites in which atherosclerosis initiates. The CZ has moderate LDL entry and saturable retention, and it develops atherosclerosis at more advanced stages of the disease as the saturation of LDL retention is gradually lost.

In normal arteries, the capacity for LDL retention is sufficient across the entire inner curvature region, but after induction of hypercholesterolemia the capacity for retaining LDL becomes saturated in the central inner zone while it continues unimpeded in the border zones. These border zones are also characterized by high levels of LDL entry and early macrophage presence, and they are the first sites to develop atherosclerotic lesions. The central inner zone is initially protected by 
atherosclerosis, but it eventually develops it as the saturation of LDL retaining mechanisms is gradually lost during disease progression. 



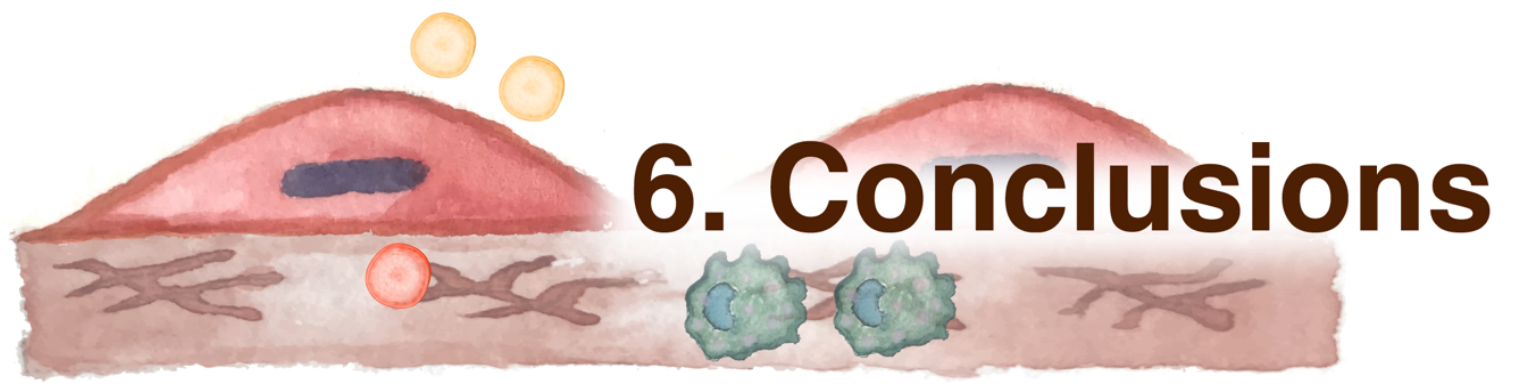


This doctoral thesis provides a detailed analysis of the rates of LDL entry and retention in the mouse aortic arch in the normal physiological state and during early atherogenesis. In summary, the following conclusions can be extracted from the experiments:

1. LDL accumulation in PCSK9-induced hypercholesterolemic mice precedes the initiation of lesions in a pre-atherosclerotic phase that marks the region that will eventually develop atherosclerosis.

2. High-resolution maps of the rates of LDL entry and retention can be achieved through in vivo injection in mice of labeled LDL followed by quantification and topography evaluation by en face infrared (IR) scanning or whole-mount confocal microscopy.

3. Rates of LDL entry and retention are dissociated in normal and preatherosclerotic mouse aortic arch. The pre-atherosclerotic aortic arch has higher rate of total labeled LDL entry but lower rate of total labeled LDL retention compared with normal arteries. Also, total LDL entry rates in the aortic arch increase during the pre-atherosclerotic phase, but this is not paralleled by similar increases in total LDL retention rates. Both observations indicate that the rate of LDL retention, not that of LDL entry, is the limiting factor for total LDL accumulation in the pre-atherosclerotic phase.

4. The differences in LDL entry and retention rates across the aortic arch divide it into three regions that respond differently to high plasma LDL levels: an outer arch zone with low LDL entry and low LDL retention, a central inner arch zone with moderate LDL entry and saturated, moderate LDL retention, and a border inner arch zone with high LDL entry and non-saturated, high LDL retention.

5. The aortic arch zones distinguished by the LDL entry and retention measurements predict the development of atherosclerosis. The border zones have high susceptibility, the central inner zone intermediate 
susceptibility and the outer zone low susceptibility to the development of atherosclerosis.

6. In the normal vasculature, the pattern of LDL entry predicts the sites that will first initiate atherogenesis if LDL levels are increased, whereas the pattern of LDL retention predicts the surface area that will eventually be covered with atherosclerotic lesions.

7. The saturation of the central inner arch zone that limits LDL accumulation in the pre-atherosclerotic phase is not explained by simple competition from high levels of plasma LDL but involves limiting retention mechanisms within the artery wall.

8. The onset of atherosclerosis is accompanied by a conversion from saturable to non-saturable LDL retention.

The study raises new questions about the mechanisms that control LDL entry and retention across the three regions of the aortic arch with differing atherosclerosis susceptibility, including in particular the retention mechanism that is saturated in some parts of pre-atherosclerotic arteries and why this limitation to LDL accumulation is lost once atherogenesis starts. 



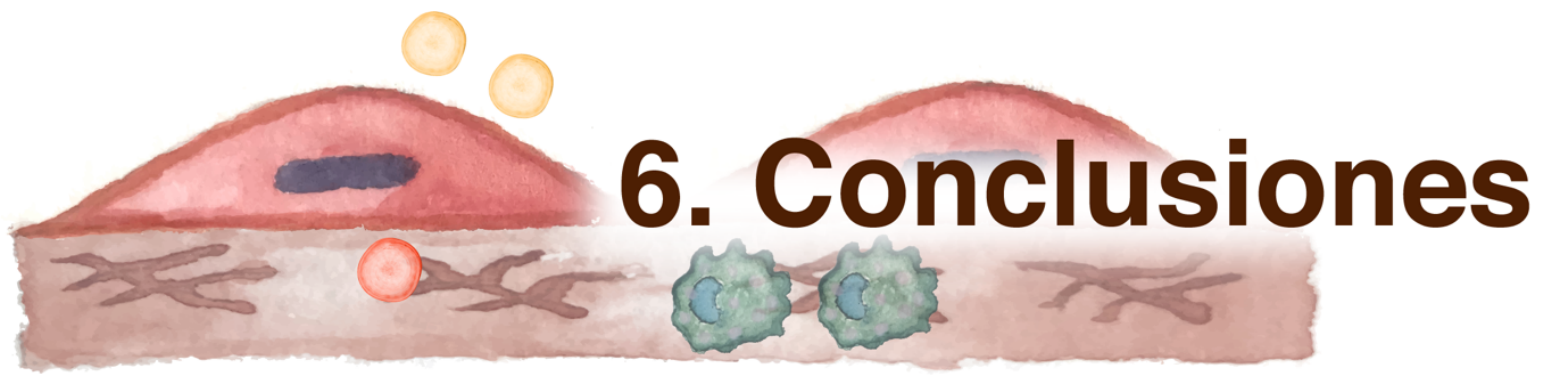


A lo largo de la presente tesis doctoral se ha desarrollado un exhaustivo análisis de las tasas de entrada y de retención de LDL en el arco aórtico del ratón, tanto en condiciones fisiológicas normales como durante estadíos tempranos de la enfermedad aterosclerótica. A continuación, se presentan las principales conclusiones extraídas a partir de los experimentos realizados:

1. La acumulación de LDL en ratones con hipercolesterolemia inducida mediante la sobreexpresión de PCSK9 precede a la iniciación de las lesiones en una fase "pre-aterosclerótica", señalando la región que terminará desarrollando la placa.

2. La inyección in vivo de ratones con partículas de LDL marcadas permite la generación de mapas de alta resolución, ilustrativos de la entrada y de la retención de dichas lipoproteínas, a través de la cuantificación y análisis topográfico mediante el escaneo en infrarrojo de muestras en face o de microscopía confocal del tejido completo.

3. Las tasas de entrada y de retención de las LDL en el arco aórtico del ratón están disociadas en la fase normal y en la pre-aterosclerótica. En esta última se encontró una tasa de entrada de LDL marcado mayor, pero menores tasas de retención que en la aorta normal. Además, la tasa de entrada en el arco aórtico de LDL totales se incrementa durante la fase preaterosclerótica, pero ello no se ve reflejado en incrementos similares en tasas de retención de LDL totales. Ambas observaciones indican que es la tasa de retención, y no la de entrada, el factor limitante para la acumulación de LDL total en la fase pre-aterosclerótica.

4. Las diferencias en la tasa de entrada y de retención de las LDL a lo largo del arco aórtico dividen a este en tres regiones por su diferente respuesta a niveles altos de LDL en plasma: una zona del arco externo con baja entrada y retención; una zona central interna del arco interno con entrada moderada de LDL, así como moderada pero saturada en su retención, y una zona fronteriza del mismo con elevada entrada de LDL y alta retención no saturada. 
5. Las regiones del arco aórtico caracterizadas por una diferente entrada y retención de partículas de LDL predicen el desarrollo de la aterosclerosis. Las regiones fronterizas poseen una elevada susceptibilidad, mientras que la zona central interna es moderadamente susceptible a estos procesos, y la externa posee una baja tendencia al desarrollo de aterosclerosis.

6. En la vasculatura normal, el patrón de entrada de las partículas de LDL sirve para predecir los sitios que prioritariamente iniciarán la aterogénesis si los niveles de LDL se incrementan, mientras que el patrón de retención es capaz de predecir el área superficial que finalmente podrá desarrollar placas de aterosclerosis.

7. La saturación de la región central del arco aórtico interno que actúa como limitante en la acumulación de LDL en la fase pre-aterosclerótica no se explica por simple competición entre elevados niveles de LDL en el plasma sanguíneo, pero sí involucra mecanismos limitantes de la retención dentro de la pared arterial.

8. El comienzo de la aterosclerosis se encuentra acompañado de una conversión del área desde una retención saturable a otra no saturable por partículas de LDL.

El presente estudio plantea nuevas cuestiones sobre los mecanismos que controlan la entrada y la retención de las partículas de LDL a lo largo de las tres regiones del arco aórtico con una diferente susceptibilidad al desarrollo de la aterosclerosis, incluyendo en particular qué mecanismos de retención se saturan en algunas regiones de la arteria pre-aterosclerótica, y por qué esta limitación de acumulación de LDL se pierde una vez la aterogénesis comienza. 



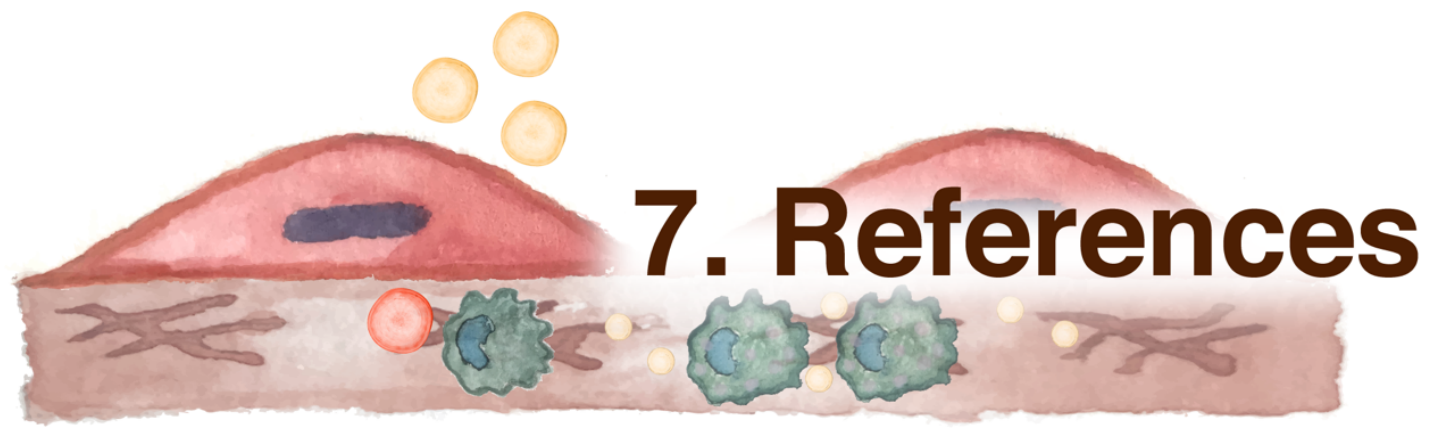


1. Bentzon JF, Otsuka F, Virmani R, Falk E. Mechanisms of plaque formation and rupture. Circ Res. 2014;114(12):1852-66.

2. Murray CJL, Lopez AD. Measuring the global burden of disease. N Engl $\mathrm{J}$ Med. 2013;369(5):448-57.

3. Lim SS, Vos T, Flaxman AD, Danaei G, Shibuya K, Adair-Rohani H, et al. A comparative risk assessment of burden of disease and injury attributable to 67 risk factors and risk factor clusters in 21 regions, 1990-2010: a systematic analysis for the Global Burden of Disease Study 2010. Lancet (London, England). 2012;380(9859):2224-60.

4. Ference BA, Ginsberg HN, Graham I, Ray KK, Packard CJ, Bruckert E, et al. Low-density lipoproteins cause atherosclerotic cardiovascular disease. 1. Evidence from genetic, epidemiologic, and clinical studies. A consensus statement fromthe European Atherosclerosis Society Consensus Panel. Eur Heart J. 2017;38(32):2459-72.

5. Foody JAM, Vishwanath R. Familial hypercholesterolemia/autosomal dominant hypercholesterolemia: Molecular defects, the LDL-C continuum, and gradients of phenotypic severity. J Clin Lipidol. 2016;10(4):970-86.

6. Wiegman A, Gidding SS, Watts GF, Chapman MJ, Ginsberg HN, Cuchel M, et al. Familial hypercholesterolæmia in children and adolescents: Gaining decades of life by optimizing detection and treatment. Eur Heart J. 2015;36(36):2425-37.

7. Steinberg D, Glass CK, Witztum JL. Evidence mandating earlier and more aggressive treatment of hypercholesterolemia. Circulation. 2008;118(6):672-7.

8. Konstantinov IE, Jankovic GM. Alexander I. Ignatowski: A pioneer in the study of atherosclerosis. Texas Hear Inst J. 2013;40(3):247-9.

9. Konstantinov IE, Mejevoi N, Anichkov NM. Nikolai N. Anichkov and his theory of atherosclerosis. Texas Hear Inst J. 2006;33(4):417-23.

10. Lee Y, Laxton V, Lin H, Chan Y, Fitzgerald-Smith S, To T, et al. Animal models of atherosclerosis (Review). Biomed Reports. 2017;6(3):259-66.

11. Borén J, Williams KJ. The central role of arterial retention of cholesterol-rich apolipoprotein-B-containing lipoproteins in the pathogenesis of atherosclerosis: a triumph of simplicity. Curr Opin Lipidol. 2016;27(5):473-83.

12. Lusis AJ. Atherosclerosis - Insight Review Articles. Nature. 2000;407(September):233-41.

13. Milutinović A, Šuput D, Zorc-Pleskovič R. Pathogenesis of atherosclerosis in the 
tunica intima, media, and adventitia of coronary arteries: An updated review. Bosn J Basic Med Sci. 2020;20(1):21-30.

14. Malek AM, Alper SL, Izumo S. Hemodynamic shear stress and its role in atherosclerosis. JAMA. 1999;282(21):2035-42.

15. Caro CG, Fitz-Gerald JM, Schroter RC. Arterial wall shear and distribution of early atheroma in man. Nature. 1969;223(5211):1159-60.

16. Peiffer V, Sherwin SJ, Weinberg PD. Does low and oscillatory wall shear stress correlate spatially with early atherosclerosis? A systematic review. Cardiovasc Res. 2013;99(2):242-50.

17. Von Der Thüsen JH, Van Berkel TJC, Biessen EAL. Induction of rapid atherogenesis by perivascular carotid collar placement in apolipoprotein Edeficient and low-density lipoprotein receptor-deficient mice. Circulation. 2001;103(8):1164-70.

18. Rippe B, Haraldsson B. Transport of macromolecules across microvascular walls: the two-pore theory. Physiol Rev. 1994;74(1):163-219.

19. Michel CC, Curry FE. Microvascular permeability. Physiol Rev. 1999;79(3):70361.

20. Fung KYY, Fairn GD, Lee WL. Transcellular vesicular transport in epithelial and endothelial cells: Challenges and opportunities. Traffic. 2018;19(1):5-18.

21. Vasile E, Simionescu M, Simionescu N. Visualization of the binding, endocytosis, and transcytosis of low-density lipoprotein in the arterial endothelium in situ. J Cell Biol. 1983;96(6):1677-89.

22. von Eckardstein A, Rohrer L. Transendothelial lipoprotein transport and regulation of endothelial permeability and integrity by lipoproteins. Curr Opin Lipidol. 2009;20(3):197-205.

23. Williams KJ, Tabas I. The response-to-retention hypothesis of early atherogenesis. Arterioscler Thromb Vasc Biol. 1995;15(5):551-62.

24. Cancel LM, Fitting A, Tarbell JM. In vitro study of LDL transport under pressurized (convective) conditions. Am J Physiol Heart Circ Physiol. 2007;293(1):H126-32.

25. Lin SJ, Jan KM, Chien S. Role of dying endothelial cells in transendothelial macromolecular transport. Arterioscler Thromb Vasc Biol. 1990;10(5):703-9.

26. Nielsen LB. Transfer of low density lipoprotein into the arterial wall and risk of atherosclerosis. Atherosclerosis. 1996;123(1-2):1-15. 
27. Zhang X, Sessa WC, Fernández-Hernando C. Endothelial Transcytosis of Lipoproteins in Atherosclerosis. Front Cardiovasc Med. 2018;5(September):130.

28. Dehouck B, Fenart L, Dehouck MP, Pierce A, Torpier G, Cecchelli R. A new function for the LDL receptor: Transcytosis of LDL across the blood-brain barrier. J Cell Biol. 1997;138(4):877-89.

29. Wiklund O, Carew TE, Steinberg D. Role of the low density lipoprotein receptor in penetration of low density lipoprotein into rabbit aortic wall. Arteriosclerosis. $1985 ; 5(2): 135-41$.

30. Armstrong SM, Sugiyama MG, Fung KYY, Gao Y, Wang C, Levy AS, et al. A novel assay uncovers an unexpected role for SR-BI in LDL transcytosis. Cardiovasc Res. 2015;108(2):268-77.

31. Calvo D, Gómez-Coronado D, Lasunción MA, Vega MA. CLA-1 is an 85-kD plasma membrane glycoprotein that acts as a high- affinity receptor for both native (HDL, LDL, and VLDL) and modified (OxLDL and AcLDL) lipoproteins. Arterioscler Thromb Vasc Biol. 1997;17(11):2341-9.

32. Huang L, Chambliss KL, Gao X, Yuhanna IS, Behling-Kelly E, Bergaya S, et al. SR-B1 drives endothelial cell LDL transcytosis via DOCK4 to promote atherosclerosis. Nature. 2019;569(7757):565-9.

33. Kraehling JR, Chidlow JH, Rajagopal C, Sugiyama MG, Fowler JW, Lee MY, et al. Genome-wide RNAi screen reveals ALK1 mediates LDL uptake and transcytosis in endothelial cells. Nat Commun. 2016;7:13516.

34. Babitt J, Trigatti B, Rigotti A, Smart EJ, Anderson RGW, Xu S, et al. Murine SR$\mathrm{BI}$, a high density lipoprotein receptor that mediates selective lipid uptake, is $\mathrm{N}$ glycosylated and fatty acylated and colocalizes with plasma membrane caveolae. J Biol Chem. 1997;272(20):13242-9.

35. Santibanez JF, Blanco FJ, Garrido-Martin EM, Sanz-Rodriguez F, Del Pozo MA, Bernabeu C. Caveolin-1 interacts and cooperates with the transforming growth factor- $\beta$ type I receptor ALK1 in endothelial caveolae. Cardiovasc Res. 2008;77(4):791-9.

36. Wang D-X, Pan Y-Q, Liu B, Dai L. Cav-1 promotes atherosclerosis by activating JNK-associated signaling. Biochem Biophys Res Commun. 2018;503(2):51320.

37. Fernández-Hernando C, Yu J, Suárez Y, Rahner C, Dávalos A, Lasunción MA, 
et al. Genetic Evidence Supporting a Critical Role of Endothelial Caveolin-1 during the Progression of Atherosclerosis. Cell Metab. 2009;10(1):48-54.

38. Ramírez CM, Zhang X, Bandyopadhyay C, Rotllan N, Sugiyama MG, Aryal B, et al. Caveolin-1 Regulates Atherogenesis by Attenuating Low-Density Lipoprotein Transcytosis and Vascular Inflammation Independently of Endothelial Nitric Oxide Synthase Activation. Circulation. 2019;140(3):225-39.

39. Schwenke DC, Carew TE. Initiation of atherosclerotic lesions in cholesterol-fed rabbits. II. Selective retention of LDL vs. selective increases in LDL permeability in susceptible sites of arteries. Arteriosclerosis. 1989;9(6):908-18.

40. FABER M. The human aorta; sulfate-containing polyuronides and the deposition of cholesterol. Arch Pathol. 1949;48(4):342-50.

41. Camejo G, Lopez A, Vegas $\mathrm{H}$, Paoli $\mathrm{H}$. The participation of aortic proteins in the formation of complexes between low density lipoproteins and intima-media extracts. Atherosclerosis. 1975;21(1):77-91.

42. Vijayagopal P, Srinivasan SR, Radhakrishnamurthy B, Berenson GS. Interaction of serum lipoproteins and a proteoglycan from bovine aorta. $J$ Biol Chem. 1981;256(15):8234-41.

43. Fogelstrand $\mathrm{P}$, Borén $\mathrm{J}$. Retention of atherogenic lipoproteins in the artery wall and its role in atherogenesis. Nutr Metab Cardiovasc Dis. 2012;22(1):1-7.

44. Wagenseil JE, Mecham RP. Vascular extracellular matrix and arterial mechanics. Physiol Rev. 2009;89(3):957-89.

45. Flood C, Gustafsson M, Richardson PE, Harvey SC, Segrest JP, Borén J. Identification of the proteoglycan binding site in apolipoprotein B48. J Biol Chem. 2002;277(35):32228-33.

46. Borén J, Olin K, Lee I, Chait A, Wight TN, Innerarity TL. Identification of the principal proteoglycan-binding site in LDL: A single-point mutation in apo-B100 severely affects proteoglycan interaction without affecting LDL receptor binding. J Clin Invest. 1998;101(12):2658-64.

47. Flood C, Gustafsson M, Pitas RE, Arnaboldi L, Walzem RL, Borén J. Molecular mechanism for changes in proteoglycan binding on compositional changes of the core and the surface of low-density lipoprotein-containing human apolipoprotein B100. Arterioscler Thromb Vasc Biol. 2004;24(3):564-70.

48. Kovanen PT, Pentikäinen MO. Decorin links low-density lipoproteins (LDL) to collagen: a novel mechanism for retention of LDL in the atherosclerotic plaque. 
Trends Cardiovasc Med. 1999;9(3-4):86-91.

49. Smith EB, Slater RS. Lipids and Low-Density Lipoproteins in Intima in Relation to Its Morphological Characteristics. In John Wiley \& Sons, Ltd; 2008 [cited 2021 Feb 26]. p. 38-62.

50. Ruuth M, Nguyen SD, Vihervaara T, Laajala TD, Uusitupa M, Schwab U, et al. Unstable LDL - Novel mechanism of atherogenesis and link to cardiovascular deaths. Atherosclerosis. 2016;252:e208.

51. Olin-Lewis K, Krauss RM, La Belle M, Blanche PJ, Hugh R Barrett P, Wight TN, et al. ApoC-III content of apoB-containing lipoproteins is associated with binding to the vascular proteoglycan biglycan. J Lipid Res. 2002;43(11):1969-77.

52. Little PJ, Ballinger ML, Osman N. Vascular wall proteoglycan synthesis and structure as a target for the prevention of atherosclerosis. Vasc Health Risk Manag. 2007;3(1):117-24.

53. Tabas I, Li Y, Brocia RW, Shu Wen Xu, Swenson TL, Williams KJ. Lipoprotein lipase and sphingomyelinase synergistically enhance the association of atherogenic lipoproteins with smooth muscle cells and extracellular matrix. A possible mechanism for low density lipoprotein and lipoprotein(a) retention and macrophage foam cell formation. J Biol Chem. 1993;268(27):20419-32.

54. Rosengren B, Jönsson-Rylander A-C, Peilot H, Camejo G, Hurt-Camejo E. Distinctiveness of secretory phospholipase A2 group IIA and V suggesting unique roles in atherosclerosis. Biochim Biophys Acta. 2006;1761(11):1301-8.

55. Camejo G, Hurt E, Wiklund O, Rosengren B, López F, Bondjers G. Modifications of low-density lipoprotein induced by arterial proteoglycans and chondroitin-6sulfate. BBA - Mol Basis Dis. 1991;1096(3):253-61.

56. Hurt-Camejo E, Camejo G, Rosengren B, López F, Ahlström C, Fager G, et al. Effect of arterial proteoglycans and glycosaminoglycans on low density lipoprotein oxidation and its uptake by human macrophages and arterial smooth muscle cells. Arterioscler Thromb Vasc Biol. 1992;12(5):569-83.

57. Miller YI, Choi S-H, Wiesner P, Fang L, Harkewicz R, Hartvigsen K, et al. Oxidation-specific epitopes are danger-associated molecular patterns recognized by pattern recognition receptors of innate immunity. Circ Res. 2011;108(2):235-48.

58. De Winther MPJ, Kanters E, Kraal G, Hofker MH. Nuclear factor kB signaling in atherogenesis. Arterioscler Thromb Vasc Biol. 2005;25(5):904-14. 
59. Libby P, Ridker PM, Hansson GK. Progress and challenges in translating the biology of atherosclerosis. Nature. 2011;473(7347):317-25.

60. Subramanian M, Tabas I. Dendritic cells in atherosclerosis. Semin Immunopathol. 2014;36(1):93-102.

61. Chistiakov DA, Melnichenko AA, Myasoedova VA, Grechko A V., Orekhov AN. Mechanisms of foam cell formation in atherosclerosis. $J \mathrm{Mol}$ Med. 2017;95(11):1153-65.

62. Chang MY, Potter-Perigo S, Tsoi C, Chait A, Wight TN. Oxidized low density lipoproteins regulate synthesis of monkey aortic smooth muscle cell proteoglycans that have enhanced native low density lipoprotein binding properties. J Biol Chem. 2000;275(7):4766-73.

63. Williams KJ, Fless GM, Petrie KA, Snyder ML, Brocia RW, Swenson TL. Mechanisms by which lipoprotein lipase alters cellular metabolism of lipoprotein(a), low density lipoprotein, and nascent lipoproteins. Roles for low density lipoprotein receptors and heparan sulfate proteoglycans. J Biol Chem. 1992;267(19):13284-92.

64. Öörni K, Hakala JK, Annila A, Ala-Korpela M, Kovanen PT. Sphingomyelinase induces aggregation and fusion, but phospholipase A2 only aggregation, of low density lipoprotein (LDL) particles: Two distinct mechanisms leading to increased binding strength of LDL to human aortic proteoglycans. J Biol Chem. 1998;273(44):29127-34.

65. Napoli C, D'Armiento FP, Mancini FP, Postiglione A, Witztum JL, Palumbo G, et al. Fatty streak formation occurs in human fetal aortas and is greatly enhanced maternal, hypercholesterolemia. Intimal accumulation of low density lipoprotein and its oxidation precede monocyte recruitment into early atheroeclerotic lesions. J Clin Invest. 1997;100(11):2680-90.

66. Stary HC. Lipid and macrophage accumulations in arteries of children and the development of atherosclerosis. Am J Clin Nutr. 2000;72(5 Suppl):1297S$1306 S$.

67. Kolodgie FD, Burke AP, Nakazawa G, Virmani R. Is pathologic intimal thickening the key to understanding early plaque progression in human atherosclerotic disease? Arterioscler Thromb Vasc Biol. 2007;27(5):986-9.

68. Otsuka F, Sakakura K, Yahagi K, Joner M, Virmani R. Has our understanding of calcification in human coronary atherosclerosis progressed? Arterioscler 
Thromb Vasc Biol. 2014;34(4):724-36.

69. Warboys CM, Amini N, De Luca A, Evans PC. The role of blood flow in determining the sites of atherosclerotic plaques. F1000 Med Rep. 2011;3(1):18.

70. Nielsen LB, Nordestgaard BG, Stender S, Kjeldsen K. Aortic permeability to LDL as a predictor of aortic cholesterol accumulation in cholesterol-fed rabbits. Arterioscler Thromb Vasc Biol. 1992;12(12):1402-9.

71. Himburg HA, Grzybowski DM, Hazel AL, LaMack JA, Li XM, Friedman MH. Spatial comparison between wall shear stress measures and porcine arterial endothelial permeability. Am J Physiol - Hear Circ Physiol. 2004;286(5 555):1916-22.

72. Steffensen LB, Mortensen MB, Kjolby M, Hagensen MK, Oxvig C, Bentzon JF. Disturbed Laminar Blood Flow Vastly Augments Lipoprotein Retention in the Artery WallSignificance. Arterioscler Thromb Vasc Biol. 2015;35(9):1928-35.

73. Stary HC, Blankenhorn DH, Chandler AB, Glagov S, Insull W, Richardson M, et al. A definition of the intima of human arteries and of its atherosclerosis- prone regions: A report from the Committee on Vascular Lesions of the Council on Arteriosclerosis, American Heart Association. Arterioscler Thromb. 1992;12(1):120-34.

74. Nerem RM, Levesque MJ, Cornhill JF. Vascular endothelial morphology as an indicator of the pattern of blood flow. J Biomech Eng. 1981;103(3):172-6.

75. Sriram K, Laughlin JG, Rangamani P, Tartakovsky DM. Shear-Induced Nitric Oxide Production by Endothelial Cells. Biophys J. 2016;111(1):208-21.

76. Nakashima Y, Fujii H, Sumiyoshi S, Wight TN, Sueishi K. Early human atherosclerosis: Accumulation of lipid and proteoglycans in intimal thickenings followed by macrophage infiltration. Arterioscler Thromb Vasc Biol. 2007;27(5):1159-65.

77. Schwenke DC, St. Clair RW. Influx, efflux, and accumulation of LDL in normal arterial areas and atherosclerotic lesions of White Carneau pigeons with naturally occurring and cholesterol-aggravated aortic atherosclerosis. Arterioscler Thromb. 1993;13(9):1368-81.

78. Tompkins RG. Quantitative analysis of blood vessel permeability of squirrel monkeys. Am J Physiol. 1991;260(4 Pt 2):H1194-204.

79. Christensen S, Stender S, Nyvad O, Bagger H. In vivo fluxes of plasma 
cholesterol, phosphatidylcholine and protein into mini-pig aortic and pulmonary segments. Atherosclerosis. 1982;41(2-3):309-19.

80. Bratzler RL, Chisolm GM, Colton CK, Smith KA, Lees RS. The distribution of labeled low-density lipoproteins across the rabbit thoracic aorta in vivo. Atherosclerosis. 1977;28(3):289-307.

81. Schwenke DC, Carew E. Quantification in vivo of increased LDL content and rate of LDL degradation in normal rabbit aorta occurring at sites susceptible to early atherosclerotic lesions. Circ Res. 1988;62(4):699-710.

82. Meir KS, Leitersdorf E. Atherosclerosis in the apolipoprotein-E-deficient mouse: a decade of progress. Arterioscler Thromb Vasc Biol. 2004;24(6):1006-14.

83. Emini Veseli B, Perrotta P, De Meyer GRA, Roth L, Van der Donckt C, Martinet W, et al. Animal models of atherosclerosis. Eur $J$ Pharmacol. 2017;816(December 2016):3-13.

84. Feingold KR. Introduction to Lipids and Lipoproteins [Internet]. Endotext. 2000.

85. Fielding CJ. Lipoprotein receptors, plasma cholesterol metabolism, and the regulation of cellular free cholesterol concentration. FASEB J. 1992;6(13):31628.

86. Beisiegel U, Weber W, Ihrke G, Herz J, Stanley KK. The LDL-receptor-related protein, LRP, is an apolipoprotein E-binding protein. Nature. 1989;341(6238):162-4.

87. Véniant $M M$, Zlot $\mathrm{CH}$, Walzem RL, Pierotti $\mathrm{V}$, Driscoll $\mathrm{R}$, Dichek $\mathrm{D}$, et al. Lipoprotein clearance mechanisms in LDL receptor-deficient "apo-B48- only" and "apo-B100-only" mice. J Clin Invest. 1998;102(8):1559-68.

88. Connelly PW. The role of hepatic lipase in lipoprotein metabolism. Clin Chim Acta. 1999;286(1-2):243-55.

89. Goldstein JL, Brown MS, Anderson RG, Russell DW, Schneider WJ. Receptormediated endocytosis: concepts emerging from the LDL receptor system. Annu Rev Cell Biol. 1985;1:1-39.

90. Johnson WJ, Mahlberg FH, Rothblat GH, Phillips MC. Cholesterol transport between cells and high-density lipoproteins. Biochim Biophys Acta. 1991;1085(3):273-98.

91. Miller NE. HDL metabolism and its role in lipid transport. Eur Heart J. 1990;11 Suppl H(SUPPL. H):1-3.

92. Marques LR, Diniz TA, Antunes BM, Rossi FE, Caperuto EC, Lira FS, et al. 
Reverse Cholesterol Transport: Molecular Mechanisms and the Non-medical Approach to Enhance HDL Cholesterol. Front Physiol. 2018;9(MAY):526.

93. Chiesa ST, Charakida M. High-Density Lipoprotein Function and Dysfunction in Health and Disease. Cardiovasc Drugs Ther. 2019;33(2):207-19.

94. Piedrahita JA, Zhang SH, Hagaman JR, Oliver PM, Maeda N. Generation of mice carrying a mutant apolipoprotein $E$ gene inactivated by gene targeting in embryonic stem cells. Proc Natl Acad Sci U S A. 1992;89(10):4471-5.

95. Zhang SH, Reddick RL, Piedrahita JA, Maeda N. Spontaneous hypercholesterolemia and arterial lesions in mice lacking apolipoprotein $E$. Science (80- ). 1992;258(5081):468-71.

96. Nakashima Y, Plump AS, Raines EW, Breslow JL, Ross R. ApoE-deficient mice develop lesions of all phases of atherosclerosis throughout the arterial tree. Arterioscler Thromb. 1994;14(1):133-40.

97. Getz GS, Reardon CA. Apoprotein E as a lipid transport and signaling protein in the blood, liver, and artery wall. J Lipid Res. 2009;50 Suppl(SUPPL.):S156-61.

98. Ishibashi S, Brown MS, Goldstein JL, Gerard RD, Hammer RE, Herz J. Hypercholesterolemia in low density lipoprotein receptor knockout mice and its reversal by adenovirus-mediated gene delivery. J Clin Invest. 1993;92(2):88393.

99. Getz GS, Reardon CA. Animal Models of Atherosclerosis. Anim Model Study Hum Dis Second Ed. 2017;32(5):205-17.

100. Bentzon JF, Falk E. Atherosclerotic lesions in mouse and man: Is it the same disease? Curr Opin Lipidol. 2010;21(5):434-40.

101. Golforoush P, Yellon DM, Davidson SM. Mouse models of atherosclerosis and their suitability for the study of myocardial infarction. Basic Res Cardiol. 2020;115(6):73.

102. Shapiro MD, Tavori H, Fazio S. PCSK9: From Basic Science Discoveries to Clinical Trials. Circ Res. 2018;122(10):1420-38.

103. Naoumova RP, Tosi I, Patel D, Neuwirth C, Horswell SD, Marais AD, et al. Severe hypercholesterolemia in four British families with the D374Y mutation in the PCSK9 gene: Long-term follow-up and treatment response. Arterioscler Thromb Vasc Biol. 2005;25(12):2654-60.

104. Berge KE, Ose L, Leren TP. Missense mutations in the PCSK9 gene are associated with hypocholesterolemia and possibly increased response to statin 
therapy. Arterioscler Thromb Vasc Biol. 2006;26(5):1094-100.

105. Bjørklund MM, Hollensen AK, Hagensen MK, Dagnæs-Hansen F, Christoffersen C, Mikkelsen JG, et al. Induction of atherosclerosis in mice and hamsters without germline genetic engineering. Circ Res. 2014;114(11):1684-9.

106. Roche-Molina M, Sanz-Rosa D, Cruz FM, García-Prieto J, López S, Abia R, et al. Induction of sustained hypercholesterolemia by single adeno-associated virus-mediated gene transfer of mutant hPCSK9. Arterioscler Thromb Vasc Biol. 2015;35(1):50-9.

107. von Scheidt M, Zhao Y, Kurt Z, Pan C, Zeng L, Yang X, et al. Applications and Limitations of Mouse Models for Understanding Human Atherosclerosis. Cell Metab. 2017;25(2):248-61.

108. Schwartz SM, DeBlois D, O'Brien ERM. The intima. Soil for atherosclerosis and restenosis. Circ Res. 1995;77(3):445-65.

109. Scandinavian Simvastatin Survival Study Group. Randomised trial of cholesterol lowering in 4444 patients with coronary heart disease: the Scandinavian Simvastatin Survival Study (4S). Lancet. 1994;344(8934):1383-9.

110. Feingold KR. Cholesterol Lowering Drugs [Internet]. Feingold KR, Anawalt B, Boyce A, Chrousos G, Dungan K, Grossman A, et al., editors. Endotext. MDText.com, Inc.; 2000.

111. Burke AC, Huff MW. Regression of atherosclerosis: lessons learned from genetically modified mouse models. Curr Opin Lipidol. 2018;29(2):87-94.

112. Reis ED, Li J, Fayad ZA, Rong JX, Hansoty D, Aguinaldo JG, et al. Dramatic remodeling of advanced atherosclerotic plaques of the apolipoprotein $\mathrm{E}$ deficient mouse in a novel transplantation model. J Vasc Surg. 2001;34(3):5417.

113. Lieu HD, Withycombe SK, Walker Q, Rong JX, Walzem RL, Wong JS, et al. Eliminating atherogenesis in mice by switching off hepatic lipoprotein secretion. Circulation. 2003;107(9):1315-21.

114. Hewing B, Parathath S, Mai CK, Fiel MI, Guo L, Fisher EA. Rapid regression of atherosclerosis with MTP inhibitor treatment. Atherosclerosis. 2013;227(1):1259.

115. Tsukamoto K, Tangirala R, Chun SH, Puré E, Rader DJ. Rapid regression of atherosclerosis induced by liver-directed gene transfer of apoE in apoE-deficient mice. Arterioscler Thromb Vasc Biol. 1999;19(9):2162-70. 
116. Li R, Chao H, Ko KWS, Cormier S, Dieker C, Nour EA, et al. Gene Therapy Targeting LDL Cholesterol but not HDL Cholesterol Induces Regression of Advanced Atherosclerosis in a Mouse Model of Familial Hypercholesterolemia. J Genet Syndr gene Ther. 2011;2(2):106.

117. Bartels ED, Christoffersen C, Lindholm MW, Nielsen LB. Altered Metabolism of LDL in the Arterial Wall Precedes Atherosclerosis Regression. Circ Res. 2015;117(11):933-42.

118. Mullick AE, Fu W, Graham MJ, Lee RG, Witchell D, Bell TA, et al. Antisense oligonucleotide reduction of apoB-ameliorated atherosclerosis in LDL receptordefi cient mice. J Lipid Res. 2011;52(5):885-96.

119. Straarup EM, Fisker N, Hedtjarn M, Lindholm MW, Rosenbohm C, Aarup V, et al. Short locked nucleic acid antisense oligonucleotides potently reduce apolipoprotein B mRNA and serum cholesterol in mice and non-human primates. Nucleic Acids Res. 2010;38(20):7100-11.

120. Schindelin J, Arganda-Carreras I, Frise E, Kaynig V, Longair M, Pietzsch T, et al. Fiji: an open-source platform for biological-image analysis. Nat Methods. 2012;9(7):676-82.

121. Schneider CA, Rasband WS, Eliceiri KW. NIH Image to ImageJ: 25 years of image analysis. Nat Methods. 2012;9(7):671-5.

122. Ruifrok AC, Johnston DA. Quantification of histochemical staining by color deconvolution. Anal Quant Cytol Histol. 2001;23(4):291-9.

123. Bogovic JA, Hanslovsky P, Wong A, Saalfeld S. Robust registration of calcium images by learned contrast synthesis. Proc - Int Symp Biomed Imaging. 2016;2016-June:1123-6.

124. Babraham Bioinformatics - FastQC A Quality Control tool for High Throughput Sequence Data [Internet].

125. Martin M. Cutadapt removes adapter sequences from high-throughput sequencing reads. EMBnet.journal. 2011;17(1):10.

126. Li B, Dewey CN. RSEM: Accurate transcript quantification from RNA-Seq data with or without a reference genome. BMC Bioinformatics. 2011;12(1):323.

127. Ritchie ME, Phipson B, Wu D, Hu Y, Law CW, Shi W, et al. Limma powers differential expression analyses for RNA-sequencing and microarray studies. Nucleic Acids Res. 2015;43(7):e47.

128. Devlin CM, Leventhal AR, Kuriakose G, Schuchman EH, Williams KJ, Tabas I. 
Acid sphingomyelinase promotes lipoprotein retention within early atheromata and accelerates lesion progression. Arterioscler Thromb Vasc Biol. 2008;28(10):1723-30.

129. Gustafsen C, Olsen D, Vilstrup J, Lund S, Reinhardt A, Wellner N, et al. Heparan sulfate proteoglycans present PCSK9 to the LDL receptor. Nat Commun. 2017;8(1):1-14.

130. Kosenko T, Golder M, Leblond G, Weng W, Lagace TA. Low density lipoprotein binds to proprotein convertase subtilisin/kexin type-9 (PCSK9) in human plasma and inhibits PCSK9-mediated low density lipoprotein receptor degradation. $J$ Biol Chem. 2013;288(12):8279-88.

131. Zeng L, Liao H, Liu Y, Lee TS, Zhu M, Wang X, et al. Sterol-responsive Elementbinding Protein (SREBP) 2 down-regulates ATP-binding cassette transporter A1 in vascular endothelial cells: A novel role of SREBP in regulating cholesterol metabolism. J Biol Chem. 2004;279(47):48801-7.

132. Bommer GT, MacDougald OA. Regulation of lipid homeostasis by the bifunctional SREBF2-miR33a locus. Cell Metab. 2011;13(3):241-7.

133. Vaisman BL, Vishnyakova TG, Freeman LA, Amar MJ, Demosky SJ, Liu C, et al. Endothelial Expression of Scavenger Receptor Class B, Type I Protects against Development of Atherosclerosis in Mice. Biomed Res Int. 2015;2015:607120.

134. Skålén K, Gustafsson M, Rydberg EK, Hultén LM, Wiklund O, Innerarity TL, et al. Subendothelial retention of atherogenic lipoproteins in early atherosclerosis. Nature. 2002;417(6890):750-4.

135. Melchior JT, Sawyer JK, Kelley KL, Shah R, Wilson MD, Hantgan RR, et al. LDL particle core enrichment in cholesteryl oleate increases proteoglycan binding and promotes atherosclerosis. J Lipid Res. 2013;54(9):2495-503.

136. Thompson JC, Tang T, Wilson PG, Yoder MH, Tannock LR. Increased atherosclerosis in mice with increased vascular biglycan content. Atherosclerosis. 2014;235(1):71-5.

137. Tang T, Thompson JC, Wilson PG, Yoder MH, Müeller J, Fischer JW, et al. Biglycan deficiency: Increased aortic aneurysm formation and lack of atheroprotection. J Mol Cell Cardiol. 2014;75:174-80.

138. She ZG, Chang $Y$, Pang HB, Han W, Chen HZ, Smith JW, et al. NG2 Proteoglycan Ablation Reduces Foam Cell Formation and Atherogenesis via 
Decreased Low-Density Lipoprotein Retention by Synthetic Smooth Muscle Cells. Arterioscler Thromb Vasc Biol. 2016;36(1):49-59.

139. Didangelos A, Mayr U, Monaco C, Mayr M. Novel role of ADAMTS-5 protein in proteoglycan turnover and lipoprotein retention in atherosclerosis. J Biol Chem. 2012;287(23):19341-5.

140. Seidelmann SB, Kuo C, Pleskac N, Molina J, Sayers S, Li R, et al. Athsq1 is an atherosclerosis modifier locus with dramatic effects on lesion area and prominent accumulation of versican. Arterioscler Thromb Vasc Biol. 2008;28(12):2180-6.

141. Bostrom MA, Boyanovsky BB, Jordan CT, Wadsworth MP, Taatjes DJ, De Beer FC, et al. Group V secretory phospholipase A2 promotes atherosclerosis: Evidence from genetically altered mice. Arterioscler Thromb Vasc Biol. 2007;27(3):600-6.

142. Gustafsson $M$, Levin $M$, Skålén $K$, Perman J, Fridén $V$, Jirholt $P$, et al. Retention of low-density lipoprotein in atherosclerotic lesions of the mouse: evidence for a role of lipoprotein lipase. Circ Res. 2007;101(8):777-83.

143. Tran-Lundmark K, Tran P-K, Paulsson-Berne G, Fridén V, Soininen R, Tryggvason $\mathrm{K}$, et al. Heparan sulfate in perlecan promotes mouse atherosclerosis: roles in lipid permeability, lipid retention, and smooth muscle cell proliferation. Circ Res. 2008;103(1):43-52.

144. Caro CG, Fitz-Gerald JM, Schroter RC. Atheroma and arterial wall shear. Observation, correlation and proposal of a shear dependent mass transfer mechanism for atherogenesis. Proc $R$ Soc London Ser B Biol Sci. 1971;177(46):109-59.

145. Cheng C, Tempel D, Van Haperen R, Van Der Baan A, Grosveld F, Daemen MJAP, et al. Atherosclerotic lesion size and vulnerability are determined by patterns of fluid shear stress. Circulation. 2006;113(23):2744-53.

146. Hajra L, Evans AI, Chen M, Hyduk SJ, Collins T, Cybulsky MI. The NF-kappa B signal transduction pathway in aortic endothelial cells is primed for activation in regions predisposed to atherosclerotic lesion formation. Proc Natl Acad Sci U S A. 2000;97(16):9052-7.

147. Weinberg PD. Rate-Limiting Steps in the Development of Atherosclerosis: The Response-to-Influx Theory. J Vasc Res. 2004;41(1):1-17.

148. van den Berg BM, Spaan JAE, Rolf TM, Vink H. Atherogenic region and diet 
diminish glycocalyx dimension and increase intima-to-media ratios at murine carotid artery bifurcation. Am J Physiol Heart Circ Physiol. 2006;290(2):H91520.

149. Mundi S, Massaro M, Scoditti E, Carluccio MA, Van Hinsbergh VWM, IruelaArispe ML, et al. Endothelial permeability, LDL deposition, and cardiovascular risk factors-A review. Cardiovasc Res. 2018;114(1):35-52.

150. Cho A, Mitchell L, Koopmans D, Langille BL. Effects of changes in blood flow rate on cell death and cell proliferation in carotid arteries of immature rabbits. Circ Res. 1997;81(3):328-37.

151. Dimmeler S, Haendeler J, Rippmann V, Nehls M, Zeiher AM. Shear stress inhibits apoptosis of human endothelial cells. FEBS Lett. 1996;399(1-2):71-4.

152. Giantsos-Adams KM, Koo AJA, Song S, Sakai J, Sankaran J, Shin JH, et al. Heparan sulfate regrowth profiles under laminar shear flow following enzymatic degradation. Cell Mol Bioeng. 2013;6(2):160-74.

153. Gouverneur M, Berg B, Nieuwdorp M, Stroes E, Vink H. Vasculoprotective properties of the endothelial glycocalyx: effects of fluid shear stress. J Intern Med. 2006;259(4):393-400.

154. Cancel LM, Ebong EE, Mensah S, Hirschberg C, Tarbell JM. Endothelial glycocalyx, apoptosis and inflammation in an atherosclerotic mouse model. Atherosclerosis. 2016;252:136-46.

155. Lewis JC, Taylor RG, Jones ND, St Clair RW, Cornhill JF. Endothelial surface characteristics in pigeon coronary artery atherosclerosis. I. Cellular alterations during the initial stages of dietary cholesterol challenge. Lab Invest. 1982;46(2):123-38.

156. Passerini AG, Polacek DC, Shi C, Francesco NM, Manduchi E, Grant GR, et al. Coexisting proinflammatory and antioxidative endothelial transcription profiles in a disturbed flow region of the adult porcine aorta. Proc Natl Acad Sci U S A. 2004;101(8):2482-7.

157. Sheikh S, Rainger GE, Gale Z, Rahman M, Nash GB. Exposure to fluid shear stress modulates the ability of endothelial cells to recruit neutrophils in response to tumor necrosis factor- $\alpha$ : A basis for local variations in vascular sensitivity to inflammation. Blood. 2003;102(8):2828-34.

158. Partridge J, Carlsen H, Enesa K, Chaudhury H, Zakkar M, Luong L, et al. Laminar shear stress acts as a switch to regulate divergent functions of NF-KB 
in endothelial cells. FASEB J. 2007;21(13):3553-61.

159. liyama K, Hajra L, liyama M, Li H, DiChiara M, Medoff BD, et al. Patterns of vascular cell adhesion molecule-1 and intercellular adhesion molecule-1 expression in rabbit and mouse atherosclerotic lesions and at sites predisposed to lesion formation. Circ Res. 1999;85(2):199-207.

160. Teferedegne B, Green MR, Guo Z, Boss JM. Mechanism of Action of a Distal NF-kB-Dependent Enhancer. Mol Cell Biol. 2006;26(15):5759-70.

161. Llodrá J, Angeli V, Liu J, Trogan E, Fisher EA, Rendolph GJ. Emigration of monocyte-derived cells from atherosclerotic lesions characterizes regressive, but not progressive, plaques. Proc Natl Acad Sci U S A. 2004;101(32):11779_ 84.

162. Corsini A, Mazzotti M, Villa A, Maggi FM, Bernini F, Romano L, et al. Ability of the LDL receptor from several animal species to recognize the human apo $B$ binding domain: studies with LDL from familial defective apo B-100. Atherosclerosis. 1992;93(1-2):95-103.

163. Kijani S, Vázquez AM, Levin M, Borén J, Fogelstrand P. Intimal hyperplasia induced by vascular intervention causes lipoprotein retention and accelerated atherosclerosis. Physiol Rep. 2017;5(14):e13334.

164. Araki M, Fan J, Watanabe T. Immunohistochemical Localization of Lipoprotein Lipase and Apolipoprotein $\mathrm{E}$ in Human Atherosclerotic Lesions. ACTA Histochem Cytochem. 1998;31(6):485-92. 